Chapman University

Chapman University Digital Commons

Fall 1-2021

\title{
Synovial Fibrosis in Osteoarthritis of the Knee: Mechanism of Development and Potential Therapeutic Targets
}

\author{
Marwa Qadri \\ Chapman University, qadri100@mail.chapman.edu
}

Follow this and additional works at: https://digitalcommons.chapman.edu/

pharmaceutical_sciences_dissertations

\section{Recommended Citation}

Qadri, M. Synovial Fibrosis in Osteoarthritis of the Knee: Mechanism of Development and Potential Therapeutic Targets. [dissertation]. Irvine, CA: Chapman University; https://doi.org/10.36837/

chapman.000208

This Dissertation is brought to you for free and open access by the Dissertations and Theses at Chapman University Digital Commons. It has been accepted for inclusion in Pharmaceutical Sciences (PhD) Dissertations by an authorized administrator of Chapman University Digital Commons. For more information, please contact laughtin@chapman.edu. 
SYNOVIAL FIBROSIS IN OSTEOARTHRITIS OF THE KNEE: MECHANISM OF DEVELOPMENT AND POTENTIAL THERAPEUTIC TARGETS

\author{
A Dissertation by \\ Marwa Qadri \\ Chapman University \\ Irvine, CA \\ School of Pharmacy \\ Submitted in partial fulfillment of the requirements for the degree of \\ Doctor of Philosophy in Pharmaceutical Sciences \\ January 2021
}

Committee in charge:

Khaled Elsaid, Pharm.D., Ph.D., Chair

Rennolds Ostrom, Ph.D.

Surya Nauli, Ph.D.

Ajay Sharma, Ph.D. 
The dissertation of Marwa Qadri is approved.

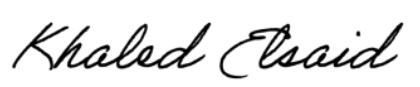

Khaled Elsaid, Pharm.D., Ph.D., Chair
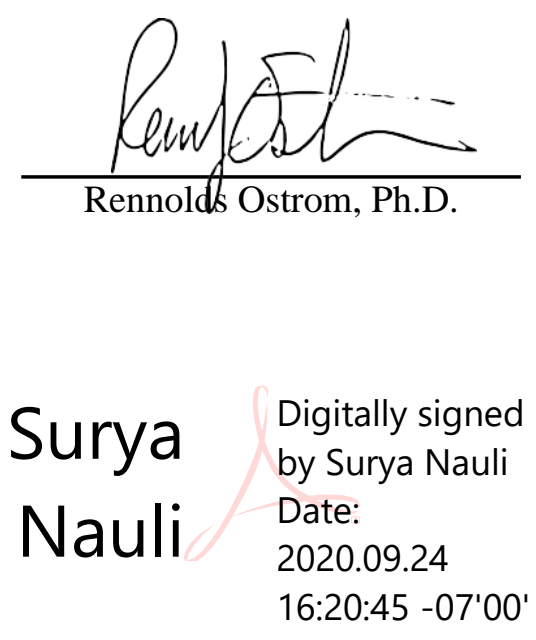

Surya Nauli, Ph.D.

Ajay Sharma

Ajay Sharma, Ph.D.

September 2020 


\section{SYNOVIAL FIBROSIS IN OSTEOARTHRITIS OF THE KNEE: MECHANISM OF DEVELOPMENT AND POTENTIAL THERAPEUTIC TARGETS}

Copyright (C) 2021

by Marwa Qadri 
In dedication to the memory of my mother who is a constant source of inspiration. Dear mother, I am extremely grateful for your love, prayers, caring, and sacrifices for educating and preparing me for my future. Thanks for teaching me that the most incredible gift to be given is love! I hope I have made you proud! 


\section{ACKNOWLEDGEMENTS}

I would like to express my special appreciation and thanks to my advisor Dr. Khaled Elsaid, who has supported me throughout my thesis with his patience, motivation and immense knowledge. Through his consistent guidance, I have been successful in conducting research and complete this dissertation. My experience with Dr. Elsaid has been absolutely fascinating and unforgettable. Dr. Elsaid always demonstrated encouragement; motivating me to pursue new ideas and to think independently. I could not have imagined having a better advisor and mentor for my $\mathrm{Ph} . \mathrm{D}$. degree. I attribute the level of my Ph.D. degree to his encouragement and effort and without him this dissertation, too, would not have been completed or written.

I would also like to thank my committee members: Dr. Rennolds Ostrom, Dr. Surya Nauli, and Dr. Ajay Sharma for their insightful comments and encouragement.

Words cannot express how grateful I am to my father and my late mother, who supported my dreams and aspirations. Thanks for all of the sacrifices that you have made on my behalf. Your prayer for me was what sustained me thus far.

I am also very much grateful to all my family members especially my sisters Bahia, Ekram, Rajaa, and Bayan and my bother Fawaz for their constant inspiration and encouragement.

I would like to express my gratitude to my close friends Bayan, Mawheba, Amani, Dania, Ali, Sandy, Mona, Eman, Shimaa, Nedaa, and Hanaa for their advice, encouragement and emotional support.

To my friend Vivian, thanks for coaching and helping me out in troubles and reaching top.

To all my friends, thank you for listening, and supporting me through this entire process.

I would like to thank my sponsors, Jazan University and Saudi Arabian Cultural Mission (SACM), for the financial support. 


\section{LIST OF PUBLICATIONS}

1-Qadri M, Jay GD, Zhang LX, Richendrfer H, Schmidt TA, Elsaid KA. Proteoglycan-4 regulates fibroblast to myofibroblast transition and expression of fibrotic genes in the synovium. Arthritis Res Ther. 2020;22(1):113.

2- Bousoik E, Qadri M, Elsaid KA. CD44 Receptor Mediates Urate Crystal Phagocytosis by Macrophages and Regulates Inflammation in A Murine Peritoneal Model of Acute Gout. Sci Rep. 2020;10(1):5748.

3-Qadri M, Jay GD, Zhang LX, et al. Recombinant human proteoglycan-4 reduces phagocytosis of urate crystals and downstream nuclear factor kappa B and inflammasome activation and production of cytokines and chemokines in human and murine macrophages. Arthritis Res Ther. 2018;20(1):192.

4-Qadri MM, Jay GD, Ostrom RS, Zhang LX, Elsaid KA. cAMP attenuates TGF- $\beta$ 's profibrotic responses in osteoarthritic synoviocytes: involvement of hyaluronan and PRG4. Am J Physiol Cell Physiol. 2018;315(3):C432-C443.

5-Qadri M, Almadani S, Jay GD, Elsaid KA. Role of CD44 in Regulating TLR2 Activation of Human Macrophages and Downstream Expression of Proinflammatory Cytokines. J Immunol. 2018;200(2):758-767. 


\section{ABSTRACT \\ SYNOVIAL FIBROSIS IN OSTEOARTHRITIS OF THE KNEE: MECHANISM OF DEVELOPMENT AND POTENTIAL THERAPEUTIC TARGETS}

by Marwa Qadri

Synovial tissue fibrosis is an immune mediated disease characterized by fibroblast cell migration, proliferation and transition to a myofibroblast phenotype characterized by alpha smooth muscle actin ( $\alpha$-SMA) upregulation and stress fiber formation. Transforming growth factor- $\beta$ (TGF- $\beta$ ) plays an important role in mediating the pathogenesis of synovial fibrosis. Synoviocytes produce proteoglycan-4 (PRG4; lubricin), a heavily glycosylated mucinous glycoprotein, and hyaluronic acid (HA). Both PRG4 and HA compete for binding to the CD44 receptor. Furthermore, rhPRG4 binds to TLR2 and TLR4 and acts as an antagonist to prevent TLR2 and TLR4 activation by bacterial ligands. In this work, we aimed to investigate the role of CD44 receptor and its ligands HA in regulating the activation of macrophages in response to TLR2 receptor stimulation in the context of an acute inflammatory response and study the impact of increasing intracellular cyclic adenosine monophosphate (cAMP) in OA fibroblast-like synoviocytes (OA-FLS) on regulating TGF- $\beta$ linked expression of fibrotic genes and the contribution of PRG4 and HA to this effect. Also, we studied the significance of PRG4-CD44 interaction in mediating fibroblast to myofibroblast transition in vitro and regulation of synovial fibrosis in vivo. In the first manuscript, we found that anti-CD44 antibody and HA treatments reduced TLR2 induced-NF- $\mathrm{BB}$ nuclear translocation, IL-1 $\beta$ and TNF- $\alpha$ expression, and production by human macrophages. In the second 
manuscript, we have shown that forskolin $(10 \mu \mathrm{M})$ increased intracellular cAMP levels and reduced TGF- $\beta$ 1-stimulated COL1A1, $\alpha$-SMA, and TIMP-1 expression in OA synoviocytes. Forskolin treatment increased HA secretion and PRG4 expression and production. Moreover, HA and PRG4 reduced $\alpha$-SMA expression and content, and PRG4 reduced COL1A1 expression and procollagen I content in OA synoviocytes. In the third manuscript, we found that rhPRG4 was internalized by OA synoviocytes via CD44 and rhPRG4 reduced the number of stress fiberpositive myofibroblasts and cell migration in TGF- $\beta$ treated NIH3T3 fibroblasts. Synovial tissues of 9-months old $\operatorname{Prg} 4^{G T / G T}$ animals had higher $\alpha$-SMA, collagen type-I and PLOD2 content and Prg4 re-expression reduced these markers. In conclusion, innate immune signals trigger macrophage activation which results in synovial inflammation. Ligand-CD44 interactions reduced the extent of innate immune-mediated macrophage activation and downstream fibroblasts' response to TGF- $\beta$ signals from immune cells. rhPRG4 may have potential therapeutic applications in mitigating synovitis and synovial fibrosis in advanced OA. 


\section{TABLE OF CONTENTS}

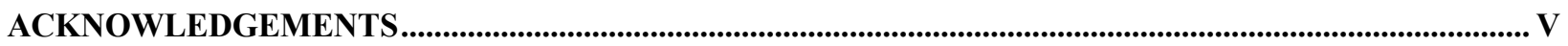

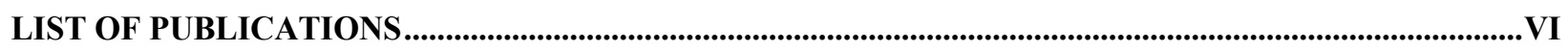

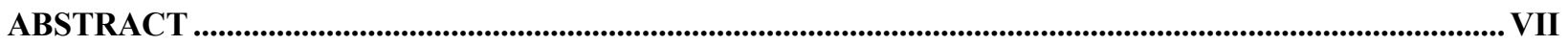

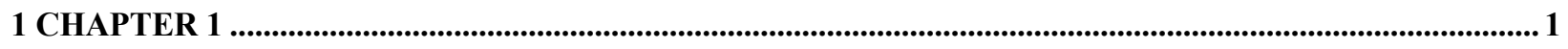

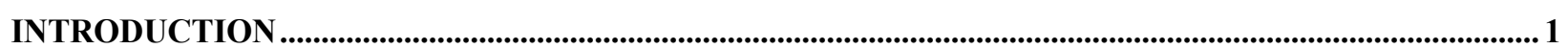

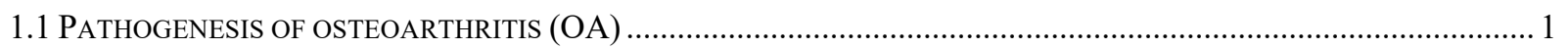

1.2 FUNCTION OF PROINFLAMMATORY AND ANTI-INFLAMMATORY CYTOKINES IN OA PATHOGENESIS..................... 3

1.3 ROLE OF THE TOLL-LIKE RECEPTORS (TLRS) IN OA PATHOGENESIS .......................................................... 5

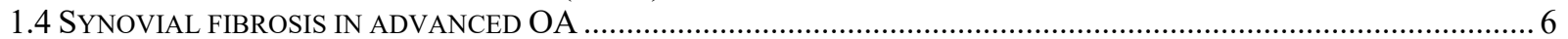

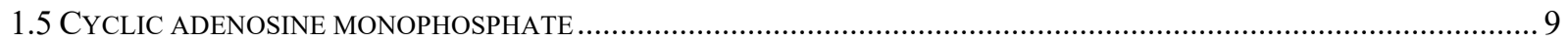

1.6 HOMEOSTATIC ROLES OF PROTEOGLYCAN-4 (PRG4) AND HYALURONAN IN THE ARTICULAR JOINT ......................... 9

1.7 CLUSTER DETERMINANT 44 (CD44) RECEPTOR AND ASSOCIATED SIGNALING PATHWAYS ................................. 11

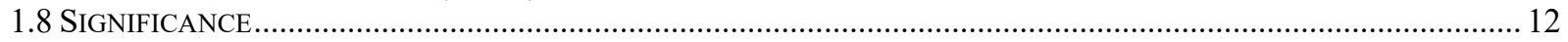

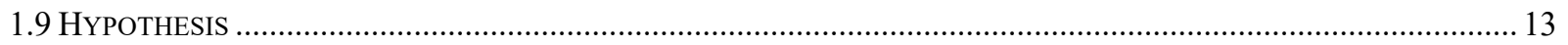

1.10 SPECIFIC AIMS:

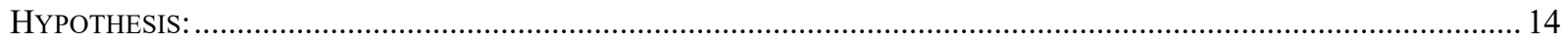

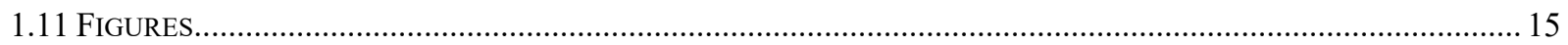

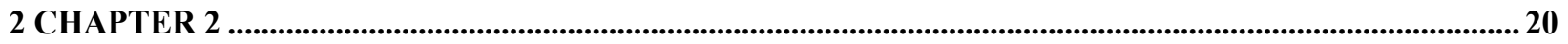

ROLE OF CD44 IN REGULATING TOLL-LIKE RECEPTOR 2 (TLR2) ACTIVATION OF HUMAN MACROPHAGES AND DOWNSTREAM EXPRESSION OF PROINFLAMMATORY CYTOKINES ........ 20

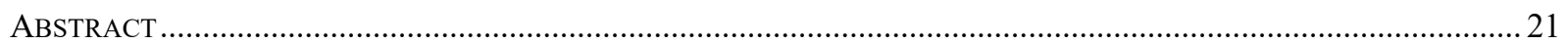

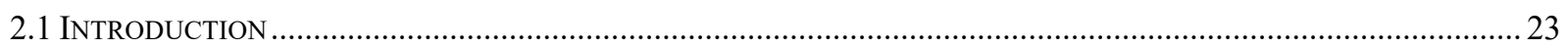

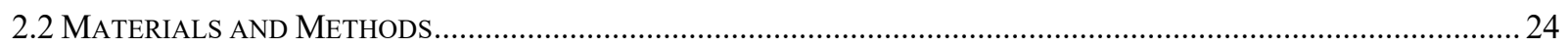

2.2.1 Differentiation of THP-1 monocytes into macrophages ..................................................................... 24

2.2.2 CD44, TLR2 and TLR4 expression in THP-1 macrophages ......................................................... 25

2.2.3 Impact of TLR2 and TLR 4 receptor activation on IL-1 $\beta$ and TNF- $\alpha$ production by THP-1 macrophages

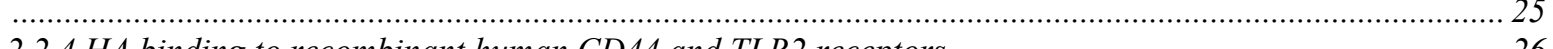

2.2.4 HA binding to recombinant human CD44 and TLR2 receptors ..................................................... 26

2.2.5 Impact of HA and CD44-specific antibody treatments on TLR2 ligand induced nuclear factor kappa $B$

$(\mathrm{NF \kappa B})$ nuclear translocation in THP-1 monocytes and macrophages ....................................................... 26

2.2.6 Impact of HA and CD44-specific antibody treatments on TLR2 ligand induced IL-1 $\beta$ and TNF- $\alpha$ gene expression and production in THP-1 macrophages ................................................................................ 27

2.2.7 Impact of HA treatment on TLR2 ligand induced CD44 expression in THP-1 macrophages ................. 28

2.2.8 CD44 receptor knockdown and associated TLR2 ligand induced IL-1 $\beta$ and TNF- $\alpha$ production in THP-1

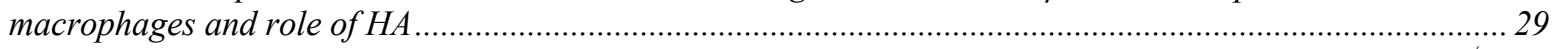

2.2.9 TLR2 ligand induced TNF- $\alpha$ production in murine bone marrow derived macrophages from $C D 44^{+/+}$

and $C D 44^{-/}$mice and effect of HA treatment on primary murine macrophages ............................................ 29

2.2.10 Activation of TLR2 and TLR4 receptors by synovial fluids from patients with OA and the role of CD44

in regulating macrophage activation in response to $O A$ synovial fluids..................................................... 31

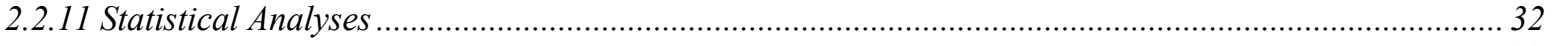

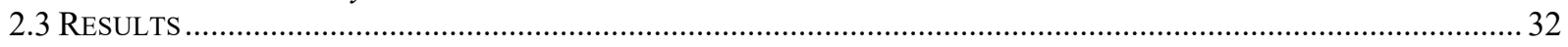


2.3.1 CD44 and TLR receptor expression in human THP-1 macrophages and proinflammatory cytokine production.

2.3.2 HA and CD44-specific antibody treatments reduced TLR2 ligand induced NFKB nuclear translocation in

THP-1 monocytes and macrophages ...................................................................................... 33

2.3.3 HA and CD44-specific antibody treatments reduced TLR2 ligand stimulated proinflammatory cytokine

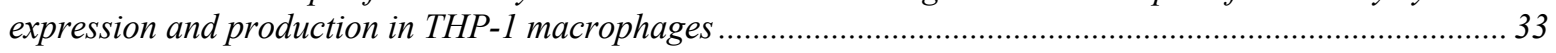

2.3.4 HA reduced CD44 expression following TLR2 receptor activation in THP-1 macrophages .................. 34

2.3.5 Role of CD44 in regulating downstream responses of TLR2 activation in THP-1 macrophages............. 35

2.3.6 CD44 modulated TLR2 ligand-induced TNF- $\alpha$ production by murine BMDMs and the HA treatment suppressed TLR2 ligand activation of primary murine BMDMs ......................................................... 35

2.3.7 OA SF specimens activated TLR2 and TLR4 receptors and a CD44-specific antibody reduced activation

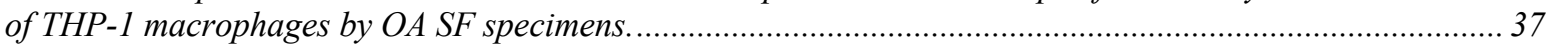

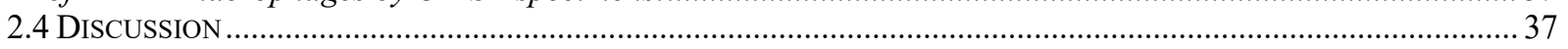

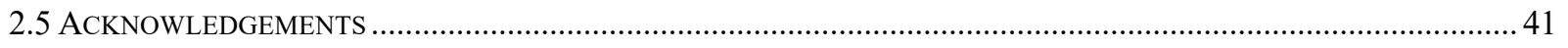

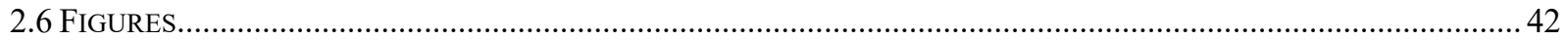

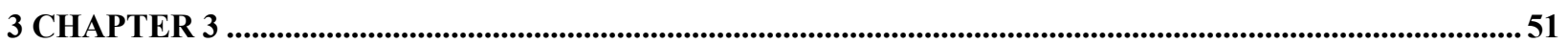

CAMP ATTENUATES TGF-B'S PROFIBROTIC RESPONSES IN OSTEOARTHRITIC SYNOVIOCYTES: INVOLVEMENT OF HYALURONAN AND PRG4 ....................................................51

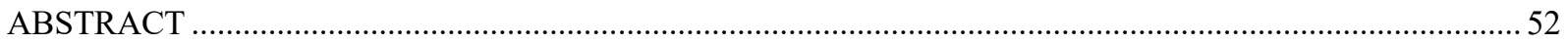

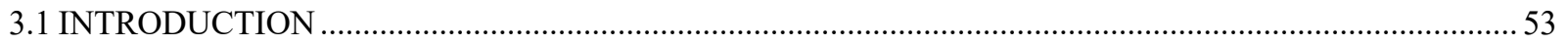

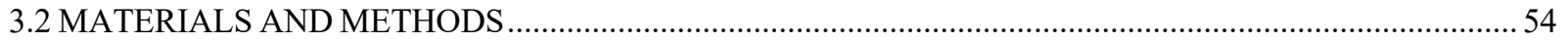

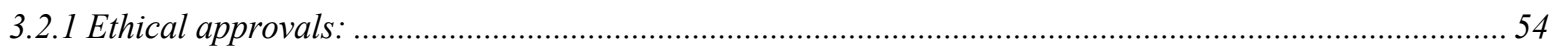

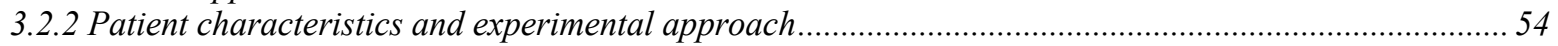

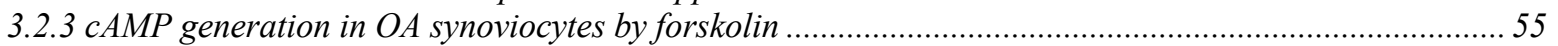

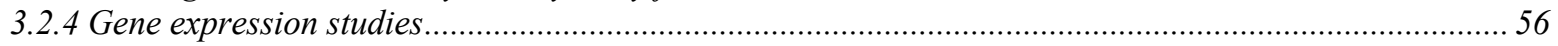

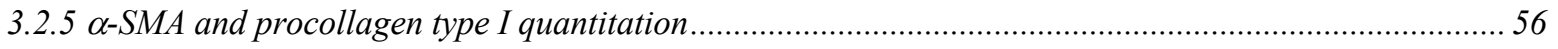

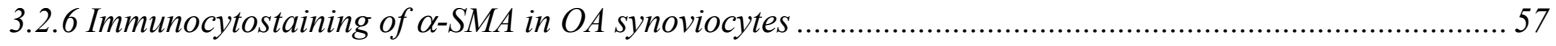

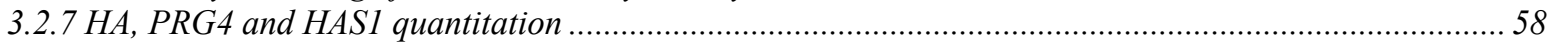

3.2.8 HAS1 knockdown and its impact on forskolin-induced HA secretion in OA synoviocytes ..................... 59

3.2.9 Gene expression studies in Prg $4^{+/+}$and $\mathrm{Prg}^{4^{-/}}$synovial tissues and immunocytostaining of murine

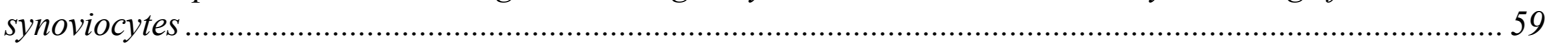

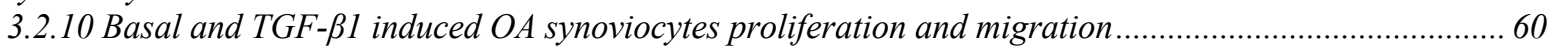

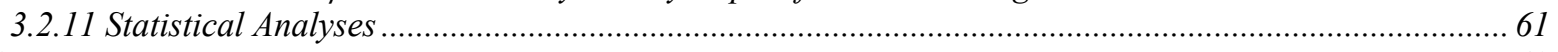

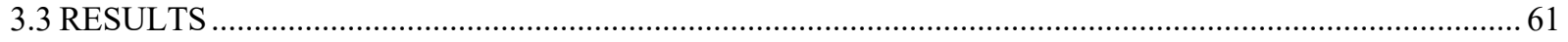

3.3.1 Forskolin treatment increased intracellular cAMP, reduced ACTA2, COL1A1, TIMP-1 expression and reduced $\alpha$-SMA and procollagen type I in TGF- $\beta 1$ stimulated $O A$ synoviocytes ........................................61 3.3.2 Forskolin treatment enhanced HA secretion and modulated HAS isoform gene expression and HAS1 knockdown attenuated forskolin's effect on HA production in TGF- $\beta 1$ stimulated OA synoviocytes............... 62 3.3.3 Forskolin treatment enhanced PRG4 expression and secretion in TGF- $\beta 1$ stimulated OA synoviocytes 63 3.3.4 Impact of PRG4 and HA treatments on ACTA2 and COL1A1 expression and $\alpha$-SMA and procollagen type I in TGF- $\beta 1$ stimulated $O A$ synoviocytes .............................................................................. 64 3.3.5 A cell permeable cAMP analog treatment reduced ACTA2 and COL1A1 expression and enhanced HA and PRG4 secretion in TGF- $\beta 1$ stimulated $O A$ synoviocytes...............................................................6 64 3.3.6 ACTA2, COL1A1 and TIMP-1 expression was higher in Prg $4^{-/}$synovial tissues and human synoviocyte PRG4 treatment reduced $\alpha-S M A$ and collagen type I staining in Prg $4^{-/}$synoviocytes ..................................65

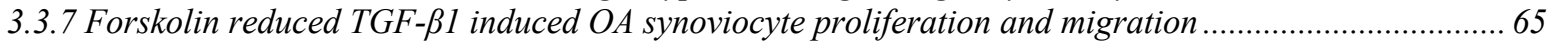

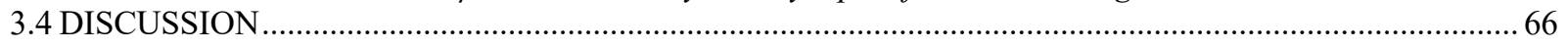

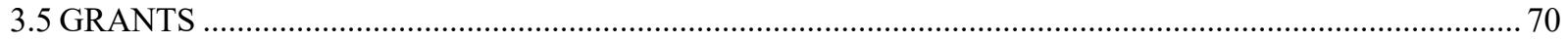

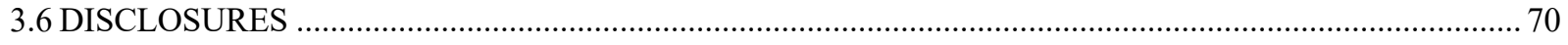

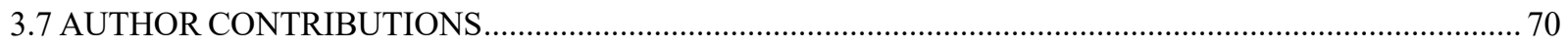

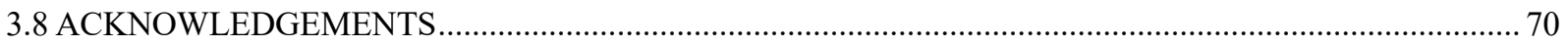

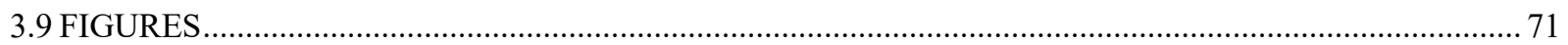


PROTEOGLYCAN-4 REGULATES FIBROBLAST TO MYOFIBROBLAST TRANSITION AND EXPRESSION OF FIBROTIC GENES IN THE SYNOVIUM .................................................................... 77

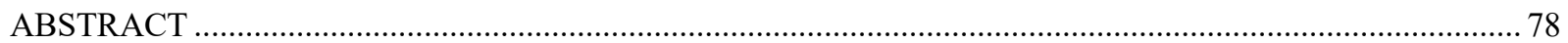

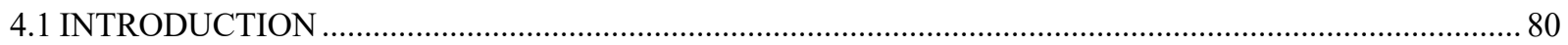

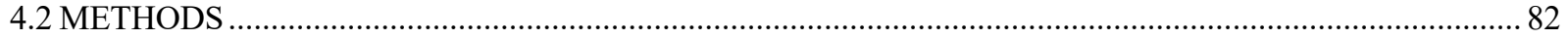

4.2.1 Impact of PRG4 and HA treatments on ACTA2 expression, $\alpha$-SMA immunostaining, and stress fiber formation in osteoarthritic fibroblast-like synoviocytes (OA FLS) and role of CD44 in mediating the effect of

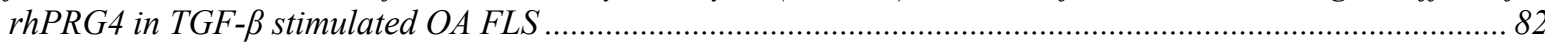
4.4.2 CD44-dependent uptake of rhPRG4, Smad3 phosphorylation and regulation of TGF- $\beta /$ Smad pathway activation in TGF- $\beta$ stimulated HEK Blue-TGF- $\beta$ cells

4.2.3 TGF- $\beta$ induction of stress fibers, focal adhesions (FAs) and cell migration in murine NIH3T3 fibroblasts, comparison of stress fibers and FAs in Prg $4^{+/+}$and Prg-/ synovial fibroblasts and role of rhPRG4 in regulating fibroblast migration

4.2.4 Generation of Active TGF- $\beta$ in lipopolysaccharide-stimulated murine macrophage J774A and NIH3T3

fibroblast co-culture and impact of rhPRG4 treatment on fibroblast migration .......................................... 87 4.2.5 Age-dependent expression of fibrotic markers in synovial tissues from Prg $4^{G T / G T}$ animals and the role of

PRG4/CD44 interaction in modulating synovial fibrosis in vivo ........................................................... 87

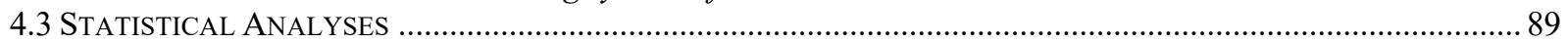

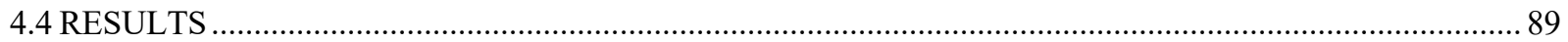

4.4.1 PRG4 reduced ACTA2 expression and stress fiber formation in osteoarthritic fibroblast-like synoviocytes (OA FLS) and CD44 was involved in the uptake of rhPRG4 by OA FLS, whereas rhPRG4-CD44

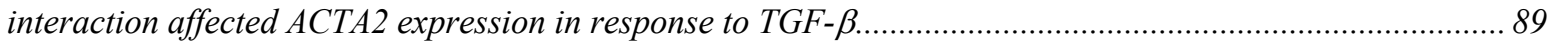
4.4.2 CD44 receptor facilitated rhPRG4 uptake by HEK-TGF $\beta$ cells and rhPRG4 inhibited TGF- $\beta 1 /$ Smad

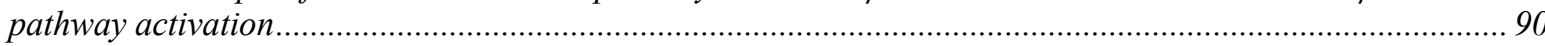
4.4.3 TGF- $\beta$ induced stress fiber and FAs formation in murine fibroblast NIH3T3 cells resulting in enhanced cell migration, and these effects were reduced with rhPRG4 treatment.....

4.4.4 Lack of Prg4 expression in murine synovial fibroblasts was associated with enhanced stress fiber formation, FA size and enhanced basal cell migration which was reduced by rhPRG4 or anti-CD44 antibody treatments.....

4.4.5 LPS stimulation of macrophages increased active and total TGF- $\beta$ levels in a macrophage and fibroblast co-culture model, induced fibroblast migration and rhPRG4 treatment reduced fibroblast migration without altering active or total TGF- $\beta$ levels.

4.4.6 Lack of Prg4 expression resulted in progressive synovial tissue fibrosis, shown by enhanced $\alpha-S M A$, collagen type-I and PLOD2 production and Prg4 re-expression reduced synovial tissue fibrosis and this effect

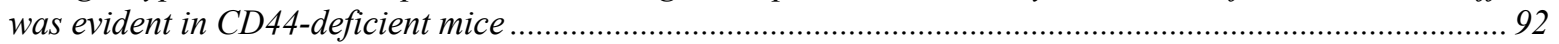

4.5 DISCUSSION

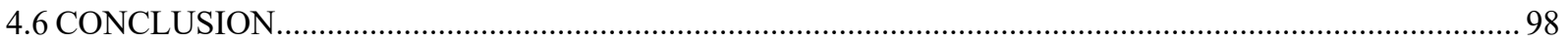

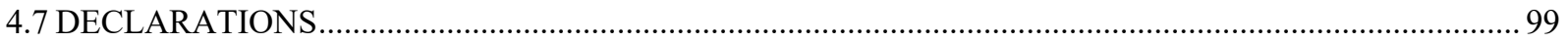

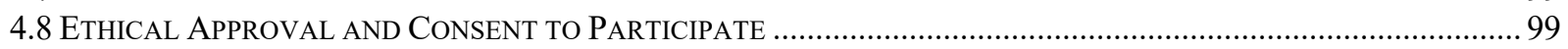

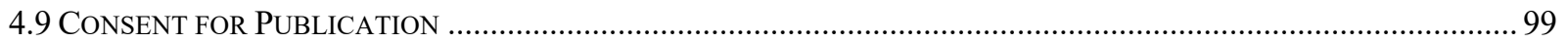

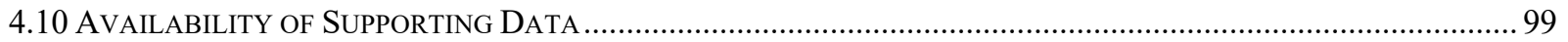

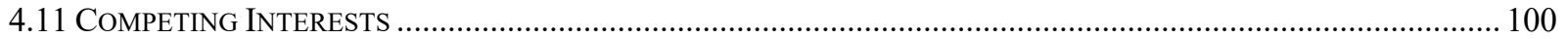

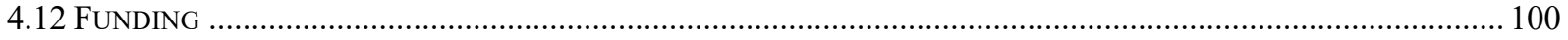

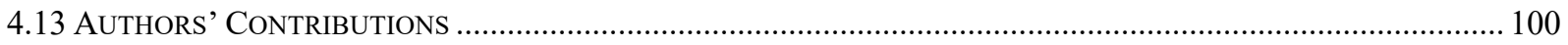

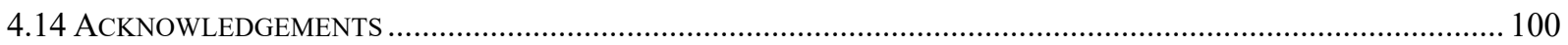

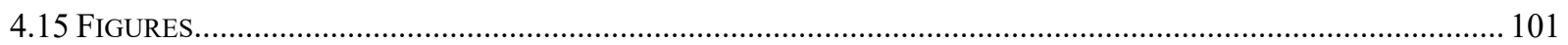

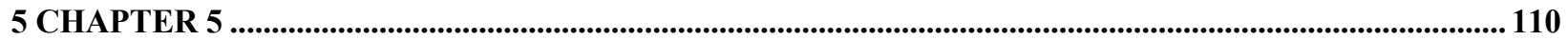

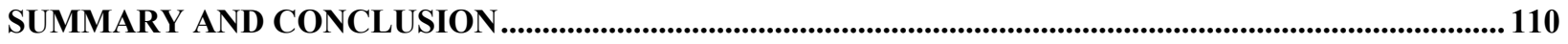

REFERENCES 


\section{LIST OF FIGURES}

Figure 1.1 Involvement of the synovium in OA pathophysiology.

Figure 1.2 The role of proinflammatory cytokines in the pathophysiology of osteoarthritis (OA).

Figure 1.3 Canonical and non-canonical TGF- $\beta$ signaling pathways.

Figure 1.4 Toll-like receptor signaling pathway.

Schematic depicting the various motifs within the 1,404 amino acid

Figure 1.5 proteoglycan-4 (PRG4) polypeptide.

Figure 2.1 Toll-like receptors 2 and 4 (TLR2 and TLR4) and CD44 receptor expression in THP-1 macrophages and impact of TLR receptor stimulation on proinflammatory cytokine production.

Figure 2.2 Binding of hyaluronan (HA) to recombinant human CD44 and TLR2 receptors and impact of HA treatment on TLR2 receptor stimulated nuclear translocation of nuclear factor kappa B (NFkB).

Figure 2.3 Impact of hyaluronan (HA) (100, 500 and 1,000 $\mu \mathrm{g} / \mathrm{mL})$, CD44 antibody (CD44 $\mathrm{Ab} ; 2.5 \mu \mathrm{g} / \mathrm{mL}$ ) or isotype control (IC; $2.5 \mu \mathrm{g} / \mathrm{mL}$ ) treatments on TLR2 receptor induced interleukin-1 beta (IL-1 $\beta$ ) or tumor necrosis factor alpha (TNF $\alpha$ ) gene expression and production in THP-1 macrophages.

Figure 2.4 Impact of toll-like receptor 2 (TLR2) receptor stimulation on CD44 gene expression and CD44 levels in THP-1 macrophages and the role of hyaluronan (HA). TLR2 receptor stimulation was performed using Pam3CSK4 (Pam; $5 \mathrm{ng} / \mathrm{mL}$ ) for 24 hours.

Figure 2.5 Impact of CD44 receptor knockdown on toll-like receptor 2 (TLR2) receptor stimulated proinflammatory cytokine production in THP-1 macrophages and nuclear factor kappa B (NFkB) p65 subunit nuclear translocation. TLR2 receptor stimulation was performed using Pam3CSK4 (Pam; 5ng/mL). 
Figure 2.6 Impact of hyaluronan (HA) treatment on toll-like receptor 2 (TLR2) induced interleukin-1 beta (IL-1 $\beta$ ) and tumor necrosis factor alpha (TNF- $\alpha$ ) production in CD44 siRNA-treated, negative control (NC) siRNA-treated and untreated control THP-1 macrophages. TLR2 stimulation was performed using Pam3CSK4 (Pam; 5ng/mL) for 24 hours.

Figure 2.7 Impact of toll-like receptor 2 (TLR2) receptor stimulation on tumor necrosis factor alpha (TNF- $\alpha$ ) production by $C D 44^{+/+}$and $C D 44^{-/}$murine bone marrow derived macrophages (BMDMs) and dose-dependent effect of HA on $C D 44^{+/+}$ BMDMs. TLR2 stimulation was performed using Pam3CSK4 (Pam; 5ng/mL) for 6 hours.

Figure 2.8 Activation of toll-like receptors 2 and (TLR2 and TLR4) by synovial fluid (SF) aspirates from patients with advanced osteoarthritis (OA) and role of CD44 in modulating THP-1 macrophages activation by OA SF.

Figure 3.1 Impact of forskolin (FsK) treatment on intracellular cyclic adenosine monophosphate (cAMP) levels, basal and transforming growth factor beta 1 (TGF- $\beta 1$ )-induced alpha smooth muscle actin (ACTA2), collagen I (COL1A1), tissue inhibitor of metalloproteinase 1 (TIMP-1) and procollagen-lysine, 2oxoglutarate 5-dioxygenase 2 (PLOD2) gene expression and alpha smooth muscle action ( $\alpha$-SMA) and procollagen type I production in osteoarthritic (OA) synoviocytes.

Figure 3.2 Impact of forskolin $(\mathrm{FsK} ; 10 \mu \mathrm{M})$ treatment on basal and transforming growth factor beta 1 (TGF- $\beta 1$ )-induced hyaluronan (HA) production, expression of hyaluronan synthase isoforms 1, 2, and 3 (HAS1, HAS2 and HAS3) and the role of HAS1 in mediating TGF- $\beta 1$ and FsK-linked HA production in osteoarthritic (OA) synoviocytes.

Figure 3.3 Impact of forskolin $(\mathrm{FsK} ; 10 \mu \mathrm{M})$ treatment on basal and transforming growth factor beta 1 (TGF- $\beta 1$ ) induced proteoglycan-4 (PRG4) gene expression and production by osteoarthritic (OA) synoviocytes and efficacy of human synoviocyte PRG4 $(100 \mu \mathrm{g} / \mathrm{ml})$ and hyaluronan $(\mathrm{HA})(100 \mu \mathrm{g} / \mathrm{ml})$ in modulating TGF- $\beta 1$ induced expression and production of alpha smooth muscle actin and collagen type I in OA synoviocytes.

Figure 3.4 Impact of 8-bromo cAMP treatment on basal and TGF- $\beta$-induced alpha smooth muscle actin (ACTA2) and collagen I (COL1A1) expression and hyaluronan (HA) and proteoglycan-4 (PRG4) secretion in osteoarthritic synoviocytes. 
Figure 3.5 Gene expression of alpha smooth muscle actin (ACTA2), collagen type I (COL1A1), tissue-inhibitor of metalloproteinase-1 (TIMP-1) and procollagenlysine, 2-oxoglutarate 5-dioxygenase 2 (PLOD2) in synovial tissues isolated from $\mathrm{Prg}^{+/+}$and $\mathrm{Prg}^{-/-}$mice and immunocytostaining of alpha smooth muscle actin ( $\alpha$-SMA) and collagen type I in $\operatorname{Prg}^{+/+}$and $\operatorname{Prg}^{4^{-/}}$synoviocytes and impact of human synoviocyte PRG4 treatment.

Figure 3.6 Impact of forskolin (FsK) treatment on basal and transforming growth factor beta 1 (TGF- $\beta 1$ ) induced proliferation and migration of osteoarthritic (OA) synoviocytes.

Figure 4.1 Impact of proteoglycan-4 (PRG4) and high molecular weight hyaluronic acid (HA) treatments on TGF- $\beta 1$ induced alpha smooth muscle actin ( $\alpha$-SMA) expression (ACTA2), production and stress fiber formation in fibroblast-like synoviocytes isolated from patients with OA (OA FLS) undergoing knee replacement surgery and $\mathrm{CD} 44$-dependent uptake of rhodamine-labeled recombinant human PRG4 (rhPRG4) into OA FLS and associated regulation of ACTA2 expression.

Figure 4.2 CD44-dependent interaction of recombinant human proteoglycan-4 (rhPRG4) with HEK Blue-TGF- $\beta$ cells and associated modulation of phosphorylated Smad3 (pSmad3) and TGF- $\beta /$ Smad signaling pathway.

Figure 4.3 Impact of recombinant human proteoglycan-4 (rhPRG4) treatment on TGF- $\beta$ induced stress fiber formation, vinculin expression, and formation of focal adhesions (FAs) in murine fibroblasts (NIH3T3) and its relationship to cell migration.

Figure 4.4 Stress fibers and focal adhesions (FAs) in murine synovial fibroblasts isolated from $\operatorname{Prg}^{-/ /}$and $\mathrm{Prg}^{+/+}$mice and their relationship to cell migration.

Figure 4.5 Impact of co-culturing murine fibroblasts (F) and murine macrophages (M) on active and total supernatant TGF- $\beta$ levels, fibroblast migration and efficacy of rhPRG4 in regulating fibroblast migration in the co-culture model.

Figure 4.6 Gene expression and immunohistological analysis of fibrotic markers: alpha smooth muscle actin ( $\alpha$-SMA) (ACTA2), collagen type-1 (COL1A1) and procollagen-lysine, 2-oxoglutarate 5-dioxygenase (PLOD2) in synovial tissues isolated from 2- and 9-months old gene-trap $\left(\operatorname{Prg} 4^{G T / G T}\right)$ and 2 and 9 months old recombined gene-trap $\left(\operatorname{Prg} 4^{\text {GTR/GTR }}\right)$ mice. 
Figure 4.7 Modulation of expression of fibrotic markers: alpha smooth muscle actin $(\alpha-$ SMA) and procollagen-lysine, 2-oxoglutarate 5-dioxygenase (PLOD2) in synovial tissues from Prg4 gene-trap animals by CD44. 


\section{LIST OF ABBREVIATIONS}

8-BrcAMP

$\mathrm{Ab}$

$\mathrm{AC}$

ACTA2

ANOVA

AP-1

ATP

BCA

BCS

BMDMs

BSA

cADDis

cAMP

CD11b

CD44

cDNA

CHO-M

COL1A1

CREB

$\mathrm{Ct}$

CTCF

DAMPs

DAPI

DMEM/F12

DNA

ELISA

F-actin

FA

FACS

FBS

$\mathrm{Fc}$ chimeric protein

FIT-C

FsK 8-bromoadenosine 3',5'-cyclic monophosphate

Antibody

Adenylyl cyclase

Actin alpha 2

Analysis of variance

Activator protein 1

Adenosine triphosphate

Bicinchoninic acid

Bovine calf serum

Bone marrow-derived macrophages

Bovine serum albumin

cAMP Difference Detector in situ

Adenosine 3',5'-cyclic monophosphate

Cluster determinant $11 \mathrm{~b}$

Cluster determinant 44

Complementary DNA

Cho hamster ovary cells

Collagen type 1

cAMP response element-binding protein

Cycle threshold

Corrected total cell fluorescence

Damage-associated molecular patterns

4',6-diamidino-2-phenylindole

Dulbecco's Modified Eagle Medium/Ham's F-12

Deoxyribonucleic acid

Enzyme-linked immunosorbent assay

Filamentous actin

Focal adhesion

Fluorescent Activated cell sorter

Fetal bovine serum

Fragment crystallizable chimeric protein

Fluorescein isothiocyanate

Forskolin 


\begin{tabular}{|c|c|}
\hline GAPDH & Glyceraldehyde 3-phosphate dehydrogenase \\
\hline GTPase & Guanosine triphosphatase \\
\hline HA & Hyaluronan \\
\hline HAS & Hyaluronan synthase \\
\hline HEK & Human embryonic kidney \\
\hline HEPES & 4-(2-hydroxyethyl)-1-piperazineethanesulfonic acid \\
\hline IC & Isotype control \\
\hline $\operatorname{IgG}$ & Immunoglobulin $\mathrm{G}$ \\
\hline IL-1 $\beta$ & Interleukin 1 beta \\
\hline IL-4 & Interleukin 4 \\
\hline ІкB & Inhibitory kappa B \\
\hline $\mathrm{kDa}$ & kilodalton (one thousand Daltons) \\
\hline LPS & Lipopolysaccharide \\
\hline M-CSF & Macrophage colony-stimulating factor \\
\hline M-PER & Mammalian protein extraction reagent \\
\hline M.w. & Molecular weight \\
\hline $\mathrm{mAb}$ & Monoclonal antibody \\
\hline MMPs & Matrix metalloproteinases \\
\hline mRNA & Messenger ribonucleic acid \\
\hline MTT & 3-(4,5-dimethylthiazol-2-yl)-2,5-diphenyltetrazolium bromide \\
\hline $\mathrm{NC}$ & Negative control \\
\hline NF-кB & Nuclear factor kappa-light-chain-enhancer of activated B cells \\
\hline NIH3T3 & NIH Swiss mouse embryo fibroblasts \\
\hline $\mathrm{OA}$ & Osteoarthritis \\
\hline OA FLS & Osteoarthritic fibroblast-like synoviocytes \\
\hline Opti-MEM & Opti-minimal essential medium \\
\hline PAGE & Polyacrylamide gel electrophoresis \\
\hline Pam & Pam3CysSerLys4 (Pam3CSK4) \\
\hline PBS & Phosphate-buffered saline \\
\hline PCR & Polymerase chain reaction \\
\hline PKA & Protein kinase A \\
\hline PLOD2 & Procollagen-lysine, 2-oxoglutarate 5-dioxygenase 2 \\
\hline PMA & Phorbol 12-myristate-13-acetate \\
\hline PP2A & Protein phosphatase $2 \mathrm{~A}$ \\
\hline PRG4 & Proteoglycan-4 \\
\hline PTOA & Posttraumatic osteoarthritis \\
\hline
\end{tabular}




$\begin{array}{ll}\text { qPCR } & \text { Quantitative polymerase chain reaction } \\ \text { RA } & \text { Rheumatoid arthritis } \\ \text { Raf-1 } & \text { Rapidly accelerated fibrosarcoma 1 } \\ \text { rhPRG4 } & \text { Recombinant human proteoglycan-4 } \\ \text { RNA } & \text { Ribonucleic acid } \\ \text { RPMI 1640 } & \text { Roswell Park Memorial Institute 1640 } \\ \text { SD } & \text { Standard deviation } \\ \text { SEAP } & \text { Secreted embryonic alkaline phosphatase } \\ \text { SF } & \text { Synovial fluids } \\ \text { siRNA } & \text { Small interfering RNA } \\ \text { Smad } & \text { Caenorhabditis elegans Sma genes and the Drosophila Mad } \\ \text { Src } & \text { Proto-oncogene c-Src (sarcoma) } \\ \text { Streptavidin-HRP } & \text { Streptavidin-horseradish peroxidase } \\ \text { TBS-T } & \text { Tris buffered saline-tween 20 } \\ \text { TGF- } \beta 1 & \text { Transforming growth factor beta 1 } \\ \text { TIMP-1 } & \text { Tissue inhibitor of metalloproteinase 1 } \\ \text { TLR } & \text { Toll-like receptor } \\ \text { TMB } & 3,3 \text { ',5,5'-Tetramethylbenzidine } \\ \text { TNF- } \alpha & \text { Tumor necrosis factor alpha } \\ \text { TRIzol } & \text { Total RNA isolation } \\ \alpha-S M A & \text { Alpha smooth muscle actin }\end{array}$




\section{CHAPTER 1 \\ INTRODUCTION}

\subsection{Pathogenesis of osteoarthritis (OA)}

Osteoarthritis (OA) is a degenerative joint disease affecting the synovium, articular cartilage and underlying bone [1]. OA is the most prevalent arthritic disease and a leading cause of disability in the elderly [1]. OA affects approximately $37.4 \%$ of adults aged 60 years or older in the United States [2]. The prevalence of $\mathrm{OA}$ is on the rise and is thought to affect more than 50 million people in the United States by the year 2020 [3]. Prevalence of OA in males is lower compared with females who tend to have a severe form of OA in age 55 and older [4]. Affected joints include knees, hips, hands as well as the spine [1]. Several risk factors have been associated with OA, including genetic predisposition, advanced age, female sex, obesity and joint trauma [4].

OA is a multifactorial disease affecting the whole joint [3]. Irreversible loss of articular cartilage, inflammation of the synovial membrane, and bone osteophytes are common features of OA [5]. A complex network of risk factors, mechanical, and biochemical parameters, including cytokines and proteolytic enzymes determine the predominance of destructive processes $[4,6,7]$. Articular cartilage is a highly specialized connective tissue and is made up of extracellular matrix (ECM) (collagen and proteoglycans) and chondrocytes $[8,9]$. Chondrocytes are responsible for the synthesis and breakdown of the ECM and the balance of chondrocytes' anabolic and catabolic processes is regulated by proinflammatory cytokines and growth factors [9]. Cytokines which have an impact on articular cartilage metabolism are classified in three groups including, catabolic (IL- 
$1 \beta$, TNF- $\alpha$ ), regulatory (IL-6, Il-8, IL-4, IL-10, IFN $\gamma$ ) and anabolic (growth factors e.g.TGF- $\beta$ ) [1, 10]. In $\mathrm{OA}$, the balance of cartilage homeostasis is shifted towards cartilage degradation. The prevailing theory of pathogenesis in $\mathrm{OA}$ is that cartilage breakdown products trigger the activation of synovial macrophages causing the release of proinflammatory cytokines, like TNF- $\alpha$, IL-1 and IL-6. These cytokines can bind to their chondrocyte receptors leading to further release of metalloproteinases and increase cartilage degradation [2].

The synovium is a soft tissue comprised of a surface layer, the intima, and an underlying subintima. The intima of normal synovium is one to three cell layers thick, with two cell types: fibroblast-like synoviocytes and macrophages, while the subintima includes blood and lymphatic vessels [8]. The synovium is responsible for the maintenance of synovial fluid by producing lubricin and hyaluronic acid [11]. Synovial tissue inflammation (synovitis) is a prominent feature contributing to OA progression. Histological changes that occur in synovium during synovitis include hyperplasia of the lining cell layer, infiltration of inflammatory cells e.g. lymphocytes and monocytes, and subintimal fibrosis $[1,2,12]$. Synovitis may be initiated by an innate immune response by synovial lining macrophages due to the release of damage associated molecular patterns (DAMPs) from articular cartilage and thus perpetuating the inflammation of the synovial membrane by synthesis of inflammatory mediators $[1,11]$ (Fig.1.1). Both synoviocytes and osteoarthritic chondrocytes produce large quantities of matrix metalloproteinases (MMPs), e.g. MMP-1, MMP-3, MMP-9 and MMP-13, and proinflammatory cytokines (IL-1 $\beta$, IL-6, TNF- $\alpha$ ), which mediate disease progression and pain associated with OA [10,13]. Accumulating evidence show that ADAMTS4 (a disintegrin-like and metalloproteinase with thrombospondin type 1 motifs), is induced upon stimulation of chondrocytes and bovine cartilage explants with IL-1 $\beta[13$, 14]. ADAMTS is family of proteins that involved in cartilage degradation in OA. Clinical 
investigations have revealed that patients with articular cartilage destruction had elevated MMP13 and ADAMTS4 expressions [2]. Moreover, transgenic mice with MMP-13-overexpression show articular cartilage destruction, suggesting that increased MMP-13 may be the cause of cartilage degradation $[15,16]$. Furthermore, double knockout of the ADAMTS4 and ADAMTS5 genes prevents articular cartilage erosion in mice $[14,17]$. These findings indicate that MMP-13 and ADAMTS4 play a crucial role in the progression of OA, and these enzymes could be potential targets in OA treatment.

Macrophages are the most common infiltrating immune cells that are present in inflamed synovial tissue $[18,19]$. Synovial macrophages play an important role in driving OA pathogenesis due to their significantly higher production of inflammatory and catabolic mediators, resulting in cartilage degeneration, synovial thickening, and osteophyte formation [19]. In addition to synovial tissue macrophages, infiltrating monocytes/macrophages may contribute to OA pathogenesis [18].

Current non-surgical treatment options focus on symptoms alleviation and these treatments don't modify the course of the underlying disease. Nonsteroidal anti- inflammatory drugs (NSAIDs) are the mainstay for moderate to severe OA [20]. Intra-articular injections of corticosteroids for acute inflammation or hyaluronic acid for persistent OA have also been shown to be effective in patients with OA [20]. Nonetheless, no disease-modifying anti-osteoarthritis drugs (DMODs) are currently available.

\subsection{Function of Proinflammatory and Anti-inflammatory cytokines in OA pathogenesis}

IL-1 $\beta$ is a very low molecular weight soluble peptide, produced and secreted in an inactive form,

pro-IL-1 $[10,13]$. IL-1 $\beta$ exists in two isoforms: IL-1alpha (IL-1 $\alpha$ ) and IL- 1beta (IL-1 $\beta$ ). IL-1 $\beta$ is one of the most important proinflammatory cytokines that mediates ECM degradation and joint 
inflammation in $\mathrm{OA}[10]$. Its role has been identified in the late 1970s as inflammatory mediator to mimic the osteoarthritic condition on cultured chondrocytes and synovial cells [13]. Previous studies have shown that IL- $1 \beta$ receptor expression was elevated in human OA chondrocytes and synovial fibroblasts compared to normal cells [10]. Binding of IL-1 $\beta$ to it specific cell-surface receptor IL-1 receptor type I (IL-1RI) initiates a signaling cascade leading to the activation of transcription factors, such as nuclear factor kappa B (NF- $\mathrm{kB})$ and interferon regulatory factors (IRFs) [21]. This signaling pathway results in a variety of cellular responses including the production of pro-inflammatory cytokines and chemokines, downregulation of the synthesis of major ECM components by inhibiting anabolic activities of chondrocytes and stimulation of chondrocytes and synovial cells to release MMPs and aggrecanases [10, 13]. Other proinflammatory cytokines that are involved in OA include TNF $\alpha$, IL-6 and IL-17 (Fig.1.2). These cytokines have been demonstrated to block chondrocytes' synthesis of type II collagen and proteoglycan and promote the production of MMPs enzymes [10].

TGF- $\beta$ belongs to a large family of growth factors with an established role in controlling cell growth, proliferation, differentiation [22]. There are three isotypes of TGF- $\beta$, called $\beta 1, \beta 2$ and $\beta 3$ [22]. TGF- $\beta$ plays an essential role in maintaining homeostasis in healthy joints. TGF- $\beta$ is secreted as an inactive complex comprised of propeptide LAP (latency associated peptide) and LTBP (latent TGF- $\beta$ binding proteins $[22,23]$. Activated TGF- $\beta$ binds to the TGF- $\beta$ type II receptor to form a complex that recruits the TGF- $\beta$ type I receptor, which is activated by phosphorylating serine/threonine kinase receptors [23]. TGF- $\beta$ signaling is initiated with ligandinduced phosphorylation of the cytoplasmic signaling molecules Smad2 and Smad3 [23]. Phosphorylated Smad2/3 form a complex with signaling transducer Smad4 and translocate to the nucleus resulting in cell-state specific modulation of transcription [23]. TGF- $\beta$ can also activate 
Erk, Jun N-terminal kinase (JNK) and p38 mitogen-activated protein kinase (MAPK) pathways [23] (Fig.1.3). TGF- $\beta 1$ plays an important role in the induction of chondrogenesis and enhancing collagen synthesis [24]. Intra-articular injection of low-dose TGF- $\beta 1$ increases proteoglycan content of articular cartilage in mouse knee joints [25]. However, overexpression of TGF- $\beta 1$ in the knee joint results in OA-like changes, including hyperplasia and synovial fibrosis [26].

\subsection{Role of the Toll-like Receptors (TLRs) in OA pathogenesis}

TLRs are large family of receptors that belong to the family of pattern recognition receptors (PRRs) [27, 28]. There are at least 11 members of TLRs that have been identified thus far [27]. TLRs play a vital role in host defenses by recognizing pathogen-associated molecular patterns (PAMPs), including cell wall components of bacteria, viruses and fungi [29]. TLRs are also stimulated by DAMPs, and endogenous danger signal molecules that are released from injured cells $[27,29]$. Examples of DAMPs that were shown to activate TLRs in the joint environment include biglycan, fibronectin and low molecular weight hyaluronan fragments, and insoluble crystals e.g. monosodium monohydrate urate and calcium phosphate crystals [30-33]. Activation of TLRs by DAMPs initiates a signaling cascade leading to the activation of transcription factors, such as NF-kB $[34,35]$. This signaling pathway results in a variety of cellular responses including the production pro-inflammatory cytokines and chemokines [35] (Fig.1.4).TLRs are transmembrane receptors characterized by an extracellular domain and a cytoplasmic Toll/IL-1 receptor (TIR) domain that initiates downstream signaling [35]. Cell surface TLRs include TLR1, TLR2, TLR4, TLR5, TLR6 and TLR10 and they mainly recognize microbial components like lipopolysaccharides [36]. Endosomal TLRs include LR3, TLR7, TLR8 and TLR9 and they recognize nucleic acids derived from bacteria and viruses [36]. 
The role of TLRs in OA pathogenesis has been studied. Chondrocytes derived from a TLR2/TLR4 double knockout mice resisted the pro-catabolic effect of low molecular weight hyaluronan and showed attenuated MMP-13 expression [37]. Furthermore, TLR2 and TLR4 can act synergistically to increase inflammatory cytokine production in OA synovial cells [38]. Early reports showed that synovial fluid (SF) from patients with early OA augments synoviocytes responses to TLR2 and TLR4-ligands and that plasma proteins present in OA SF stimulate cytokine production in a TLR4-dependent manner [39]. Additionally, it has been recently observed that OA SF activates TLR2 and TLR4 on human embryonic kidney cells [40].

Moreover, the role of innate immune system in mediating tissue remodeling and fibrosis has been highlighted in a number of studies. Recent studies showed that activation of TLR4 sensitizes skin fibroblasts to the stimulatory effect of TGF- $\beta 1$ and enhances the accumulation of ECM molecules [41]. Moreover, fibroblasts isolated from $T L R 4^{-/}$mice showed attenuated profibrotic activities of TGF- $\beta 1$, suggesting the innate immune signaling via TLRs are essential for fibroblast activation [41]. Interestingly, synovial resident cells have been shown to express TLRs and persistent inflammation might play a considerable role in the development of synovial fibrosis.

\subsection{Synovial fibrosis in advanced OA}

Major abnormalities observed in the OA synovium include synovial hyperplasia, inflammatory cell infiltration, angiogenesis and fibrosis [11]. Fibrosis is a non-physiological wound healing process that is characterized by excessive deposition of ECM proteins, in particular collagens coupled with reduced turnover [42]. Synovial fibrosis is a common feature in advanced OA that contributes to joint pain and stiffness [42]. Synovial fibrosis is a prevalent outcome of total knee arthroplasty (TKA) where the collagen-rich dense fibrous tissue limits the normal range of motion 
and contributes to functional deficits [43]. Patients with synovial fibrosis are more likely to experience a first and follow-up TKA and reducing the rate of fibrosis may reduce the likelihood of a revision TKA [44]. The underlying mechanism that causes synovial fibrosis has not been investigated.

The TGF- $\beta$ signaling pathways play an essential role in maintaining joint homeostasis in healthy joints, and a switch to a pathologic role that drives synovial fibrosis [45]. Blaney et al showed that intra-articular injection of adenovirus overexpressing active TGF- $\beta$ in the knee joints resulted in a significant increase of synovial thickness 2 weeks after injection [46]. Additionally, previous observations showed that TGF- $\beta$ upregulates the expression of synovial collagen type 1 , tissue inhibitor of metalloproteinase 1 (TIMP-1), and procollagen-lysine, 2-oxoglutarate 5-dioxygenase 2 (PLOD2) and promotes the differentiation of OA synovial fibroblasts into a myofibroblast-like phenotype [47]. Myofibroblasts have enhanced ability to express alpha smooth muscle actin $(\alpha-$ SMA) and produce collagen, proliferate and migrate [47, 48].

Synovial macrophages play an important role in driving OA pathogenesis due to their significantly higher production of inflammatory and catabolic mediators, resulting in cartilage degeneration, synovial thickening, and osteophyte formation [19]. In addition, local depletion of synovial macrophages was shown to mitigate cartilage damage and osteophyte formation in experimental OA models [49]. Using a macrophage Fas-induced apoptosis (MaFIA) transgenic mouse, clodronate liposomes-mediated depletion of synovial lining macrophages was shown to limit cartilage damage and synovial inflammation [49]. In addition to synovial tissue macrophages, infiltrating monocytes/macrophages may contribute to OA pathogenesis [18]. Monocytes and macrophages are found with increased numbers in arthrofibrotic tissues, which may signal their 
involvement in the pathogenesis of synovial fibrosis [50]. Furthermore, chemotactic factors e.g. IL-8 and MCP-1 were highly expressed in fibrotic synovial tissues, infrapatellar fat pad, and SF from patients with revision TKA compared to patients with non-fibrotic primary TKA [43, 50, 51].

Moreover, inflammatory microenvironment in OA synovium has been implicated in switching both synovial-resident and infiltrating monocytes/macrophages into two main subsets M1 (proinflammatory) to M2 (anti-inflammatory) [52]. Following tissue injury, monocytes migrate and differentiate into M1 inflammatory macrophages [53]. Activation of M1 macrophages is achieved by inflammatory cytokines e.g. IFN- $\gamma$ and via the toll-like receptor system $[52,54]$. M1 macrophages produce TGF- $\beta$ as a regulatory feedback signal to aid in the resolution of inflammation $[54,55]$. One of the first steps following tissue injury is fibroblasts migration, differentiation into myofibroblasts and production of matrix proteins in response to TGF- $\beta$ [55, 56]. M2 macrophages are anti-inflammatory by virtue of producing IL-10 and TGF- $\beta$, and in this regard antagonize the profibrotic role of M1 macrophages [57]. Excessive M2 macrophage responses can contribute to pathologic fibrosis via increased production of profibrotic mediators e.g. TGF- $\beta[57,58]$. Our understanding of the contribution of M1 and M2 macrophages to OA synovitis and synovial fibrosis remains limited. Available evidence supports that synovial macrophage M1 polarization exacerbated synovitis and resolution of synovial inflammation was associated with the population of the synovium by M2 macrophages that possess a homeostatic anti-inflammatory phenotype [59,60]. Furthermore, analysis of aspirates collected from patients with advanced OA shows that the concentrations of CD163 and CD14, M2 macrophage soluble markers, were higher in synovial fluid and plasma in these patients [61]. These data suggest that monocytes/macrophages may play a role in regulating synovitis and ensuing synovial fibrosis. 


\subsection{Cyclic adenosine monophosphate}

Cyclic adenosine monophosphate is a pleiotropic intracellular second messenger generated by adenylyl cyclase enzymes in response to G-protein coupled receptor (GPCR) activation [62]. The antifibrotic effect of cAMP has been shown in fibroblasts from multiple origins and includes inhibition of fibroblast proliferation, fibroblast-myofibroblast transition, reduction in fibroblast migration and synthesis of ECM components $[63,64]$. It has been shown that isoproterenol, a cAMP-elevating agent, inhibited TGF- $\beta$-stimulated collagen synthesis and $\alpha$-SMA expression in cardiac fibroblasts [63]. Also, stimulation of cAMP production blunted TGF- $\beta$-induced pulmonary fibroblasts proliferation [64]. The increase in cAMP results in activation of protein kinase A (PKA)-dependent and -independent pathways $[65,66]$. In the presence of growth factors, cAMP elevation activates cAMP-dependent PKA, which interferes with Raf-1 activation and signaling to blunt cell proliferation [67]. PKA also activates CREB, which can compete for cofactors with SMAD- mediated transcription stimulated by TGF- $\beta 1$ [63]. In the absence of growth factors, PKAdependent and independent pathways stimulate cAMP-mediated cell proliferation [68]. However, the role of cAMP in controlling TGF- $\beta$ fibrotic response in osteoarthritic synovial fibroblasts remains unknown.

\subsection{Homeostatic roles of proteoglycan-4 (PRG4) and hyaluronan in the articular joint}

Lubricin/Proteoglycan 4 (PRG4) is a glycoprotein secreted from synovial fibroblasts and superficial zone chondrocytes and has a multifaceted function including boundary lubrication, friction lowering of apposed cartilage surfaces and prevention of synovial overgrowth [69-72]. PRG4 is abundant in synovial fluid (SF) and PRG4 SF levels are reduced following acute joint trauma and in patients with osteoarthritis and rheumatoid arthritis (RA) [73, 74]. In animal models 
of posttraumatic osteoarthritis (PTOA) and inflammatory arthritis, Prg4 expression is reduced in cartilage and synovium [75-77]. Cartilage biosynthesis of PRG4 is reduced as a result of IL-1 $\beta$ and TNF $\alpha$ exposure but is increased with TGF $\beta[78,79]$. In synoviocytes, Prg4 expression is reduced by IL-1 $\beta$ and is increased by TGF $\beta$ and TGF $\beta$-linked PRG4 accumulation is counterbalanced by IL-1 $[79,80]$. Loss of function mutations in the Prg4 gene is evident in the autosomal recessive disease Camptodactylyl-Arthropathy-Coxa Vara Pericarditis (CACP) syndrome which is a juvenile-onset arthropathy [81]. This phenotype is recapitulated in the Prg4 knockout $\left(\operatorname{Prg}^{-/-}\right)$mouse, which displays progressive synovial hyperplasia, cartilage surface fibrillations and damage [81]. PRG4's joint homeostatic role is further evidenced by the ability of purified human PRG4, recombinant full-length or truncated PRG4 to retard cartilage degeneration, enhance cartilage repair and reduce chondrocyte apoptosis in PTOA models [82]. PRG4's protein core is 1,404 amino acids long with globular $\mathrm{N}$ and $\mathrm{C}$ termini and a central mucin domain (Fig. 1.5). The central mucin domain is heavily glycosylated via O-linked $\beta \quad 1-3)$ Gal-GalNAc oligosaccharides, and is configured to form a nanofilm that exerts repulsive forces, and provides the basis for its anti-adhesive and lubricating properties [83]. Removal of the saccharides from PRG4 results in a loss of boundary lubricating ability, while the C-terminus may facilitate surface binding of the protein [83]. The specific pattern of glycosylation may play a role in mediating PRG4's biological functions [84]. PRG4 from RA SF exhibits increased siyalylation potentially leading to an altered glycosylation-dependent biological role for PRG4 [85]. PRG4 is shown to bind to L-selectin in a glycosylation-dependent manner and coats polymorphonuclear granulocytes recruited to inflamed synovia and SF of patients with RA [84, 85]. PRG4 was shown to bind the CD44 receptor in a concentration-dependent manner and competes with high-molecular weight hyaluronan, a CD44 ligand, on binding to CD44 [86]. rhPRG4-CD44 interaction mediates 
rhPRG4's ability to inhibit IL-1 $\beta$ and TNF $\alpha$-induced proliferation of fibroblast-like synoviocytes from patients with RA. The downstream effect of engaging the CD44 receptor by rhPRG4 is blocking NF-kB nuclear translocation [86]. Also PRG4 was shown to bind TLR2 and TLR4 receptors and acts as an antagonist to prevent TLR2 and TLR4 activation by bacterial ligands [40].

HA, a major ECM glycosaminoglycan, is a high molecular-weight polysaccharide [87]. HA is produced by fibroblast-like synoviocytes and synthesized as high-molecular weight (HMW) forms (MW, $>1000 \mathrm{kDa}$ ) by HA synthase (HAS) with three isoforms identified to date (HAS1, HAS2, and HAS3) [88]. HA is a primary lubricant molecule in synovial fluid with concentration ranges 1 to $4 \mathrm{mg} / \mathrm{mL}$ [87]. HA mediates its biological functions through binding to transmembrane receptor, CD44 [89]. Recent studies showed that high molecular weight hyaluronic acid (HMWHA) suppressed IL-1 $\beta$-mediated NF- $\kappa B$ activation and downstream expression of MMP-1 and MMP-3 in OA synovial cells in a CD44-dependent manner [90]. Additionally, HA's antiinflammatory effects may be mediated by its ability to modulate TLR2 and TLR4 cartilage expression in experimental arthritis [91]. In addition, HA play an important role in joints fibrosis, as intraarticular injection of HA reduced TGF- $\beta 1$-induced synovial fibrosis in a murine model of OA [92].

\subsection{Cluster determinant 44 (CD44) receptor and associated signaling pathways}

CD44 is a transmembrane glycoprotein, with various isoforms generated by alternative splicing, plays a crucial role in cell adhesion, development, endocytosis, tissues homeostasis, and in a variety of inflammatory diseases [93]. CD44 has an established role in hyaluronan metabolism and cellular uptake [89, 93]. CD44 was shown to bind to osteopontin, collagen, and MMPs and different ECM components including PRG4, HA [94]. 
Previous studies have suggested that CD44 has role in OA development. CD44 expression was detected in synovia and cartilage of patients with different severities of OA [95]. CD44 receptor has a role in regulating cell signaling pathways. A study by Rothman et al demonstrated that antiCD44 mAb elevates cAMP and inhibits the increase in intracellular $\mathrm{Ca}^{2+}$ in human $\mathrm{T}$ lymphocytes [96]. It was suggested that CD44 might be positively coupled with adenylyl cyclase and that activation of adenylyl cyclase by anti-CD44 mAb or forskolin, an adenylyl cyclase activator, may inhibit the increase in intracellular $\mathrm{Ca}^{2+}$ in human $\mathrm{T}$ lymphocytes [96]. In the context of innate immunity, CD44 was found to regulate Fc $\gamma$ and complement receptor 3-dependent macrophage phagocytosis $[93,97]$. Additionally, CD44 may play a role in the negative regulation of TLR receptor activation [98]. Furthermore, CD44 engagement by the antibody resulted in PP2A activity activation and inhibition of PP2A activity revered the anti-inflammatory effect of the antibody [99]. In addition to its role in mediating cellular migration and inflammation, CD44 receptor contributes to fibrosis in response to injury. $C D 44^{+/+}$fibroblasts produce more active TGF- $\beta 1$ than $C D 44^{-/-}$fibroblasts [100], suggesting an effect of CD44 targeting in suppressing the activation of fibroblasts.

\subsection{Significance}

OA fibrosis is a common outcome of advanced OA that contributes to joint pain, stiffness and functional deficits. OA fibrosis is an immune mediated disease characterized by fibroblast cell migration, proliferation and transition to a myofibroblast phenotype characterized by alpha smooth muscle actin ( $\alpha$-SMA) upregulation and stress fiber formation. Synoviocytes produce proteoglycan-4 (PRG4; lubricin), a heavily glycosylated mucinous glycoprotein, and hyaluronic acid (HA). PRG4 is abundant in synovial fluid (SF) and PRG4 SF levels are reduced following 
acute joint trauma and in patients with OA. PRG4 play an important role in joint lubrication and it binds to CD44, the HA receptor, to exert an anti-inflammatory effect in the articular joint. The role of synovial PRG4 in the context of resolution of synovitis and development of fibrosis remains unknown. Moreover, the pathological mechanisms that cause OA fibrosis are unclear, and current treatments include physical therapy and revision surgeries. Given that OA fibrosis develops in $~ 3$ $10 \%$ of patients with total knee arthroplasty (TKA), there is a significant need for new prophylactic and/or treatment modalities. This study expands our understanding of the pathophysiology of OA fibrosis and provides us with the rationale to pursue PRG4 as a promising biologic treatment to prevent and/or treat OA fibrosis.

\subsection{Hypothesis}

PRG4 in synovial fluid possesses an anti-inflammatory and antifibrotic effect that prevents immune-mediated TGF- $\beta$ activation and OA fibrosis is associated with a reduction in PRG4's biological effect.

\subsection{Specific Aims:}

We propose three interconnected aims to test our overall hypothesis:

Aim 1: To evaluate the role of CD44 in regulating NF- $\kappa B$ activation and proinflammatory cytokine production in response to TLR2 receptor activation in human macrophages using a combination of CD44 receptor knockdown, TNF- $\alpha$ production by macrophages from CD44 wildtype and knockout mice, and CD44 receptor engagement by CD44-neutralizing antibody and HA treatments. 


\section{Hypothesis:}

CD44 regulates TLR2 activation of human macrophages, whereby a reduction in CD44 levels or engagement of CD44 by its ligand (HA) or CD44-specific antibody reduces downstream proinflammatory cytokine production.

Aim 2: To investigate the antifibrotic function of cAMP, generated by forskolin treatment, in TGF$\beta$ stimulated OA synoviocytes and study the contribution of synovial fluid components PRG4 and HA to OA fibrosis.

Hypothesis: Increasing intracellular cAMP exerts an antifibrotic effect in OA synovial fibroblasts and promotes PRG4 and HA secretion.

Aim 3: To examine the role of PRG4-CD44 interaction in regulating synovial tissue fibrosis in vitro and in vivo.

Hypothesis: PRG4 regulates fibroblast to myofibroblast transition and modulates fibroblast migration in response to exogenous TGF- $\beta$ or co-incubation with lipopolysaccharide (LPS) stimulated macrophages in a CD44-dependent manner. 


\subsection{Figures}

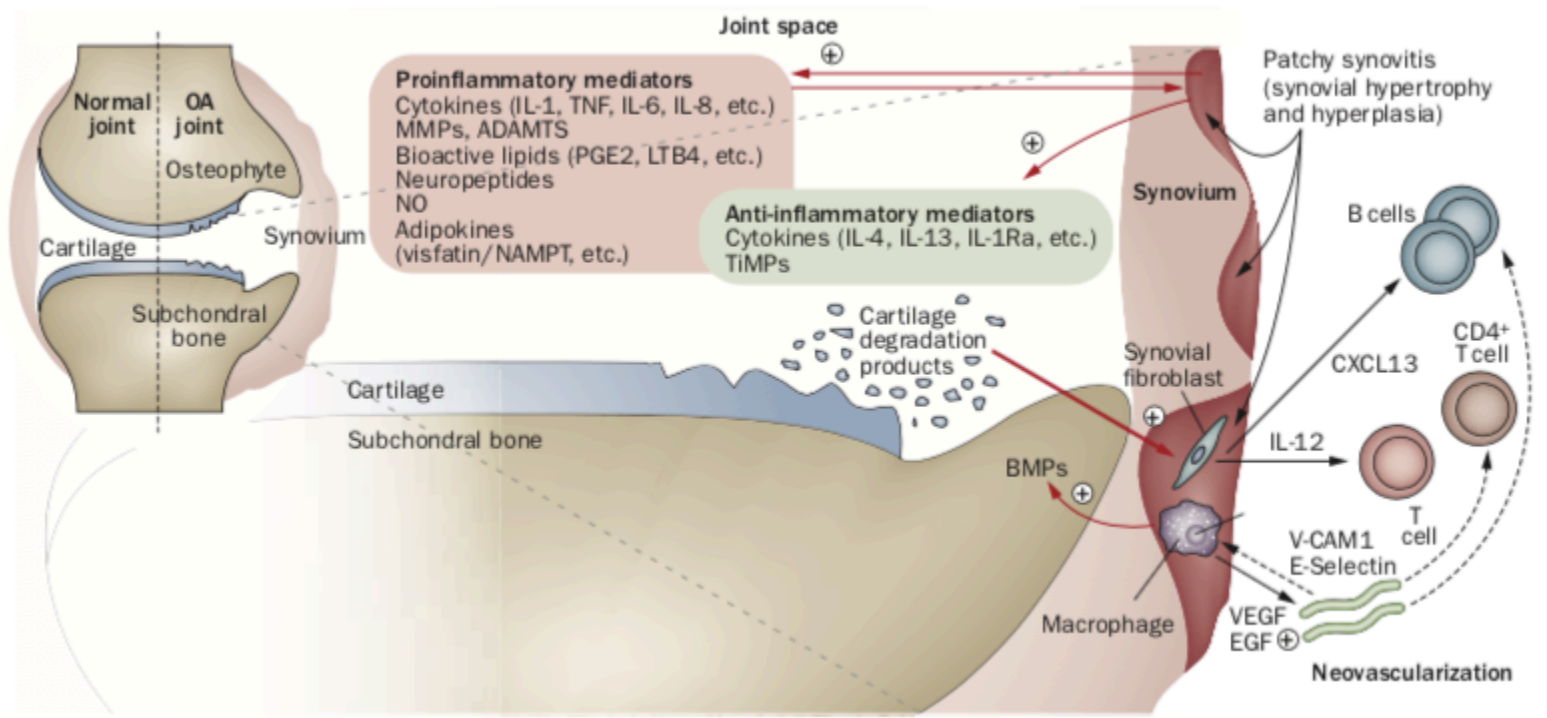

Figure 1.1 Involvement of the synovium in OA pathophysiology. Products of cartilage breakdown that are released into the synovial fluid are phagocytosed by synovial cells, amplifying synovial inflammation [12]. 


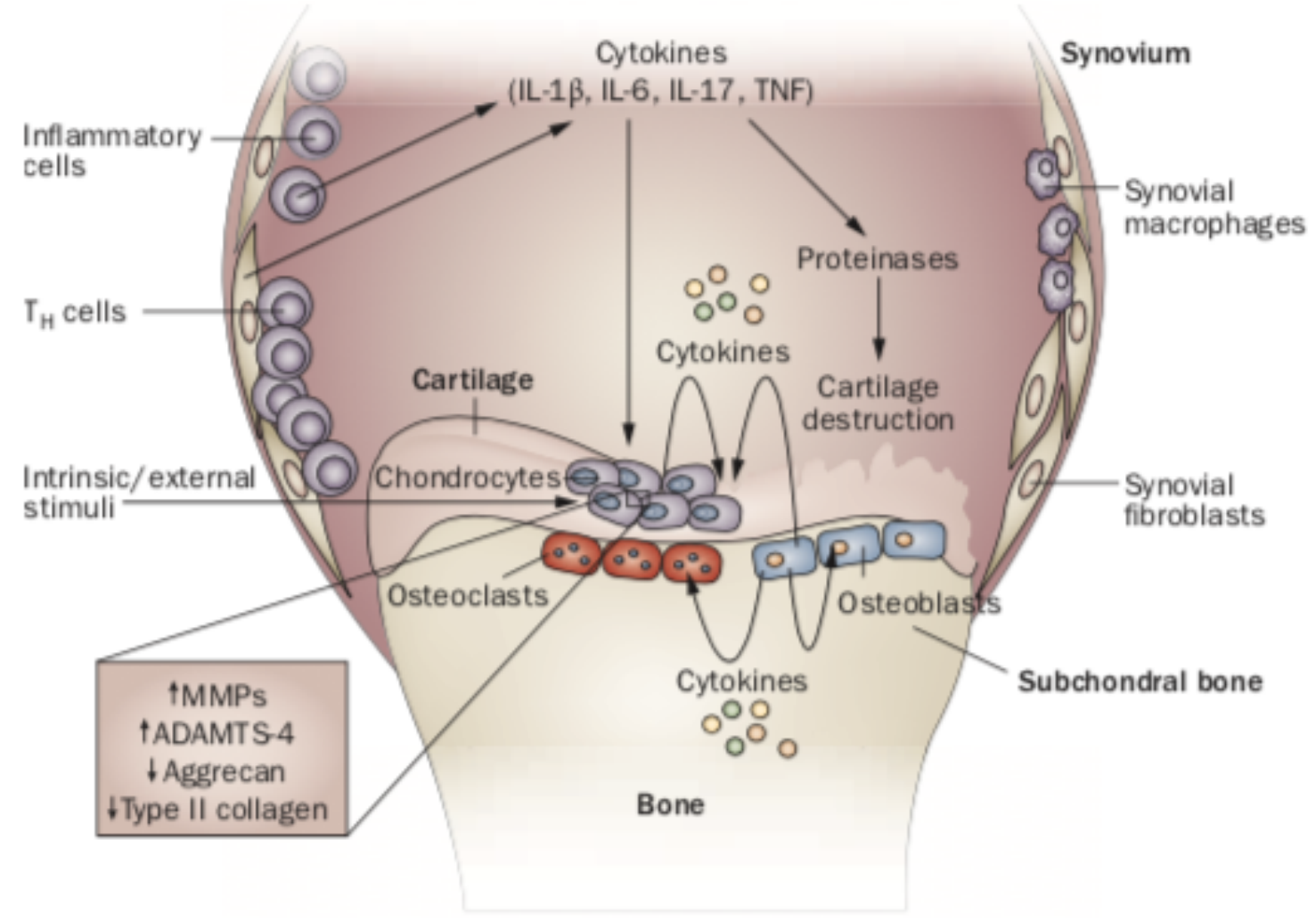

Figure 1.2 The role of proinflammatory cytokines in the pathophysiology of osteoarthritis (OA). The levels of proinflammatory cytokines, including IL-1 $\beta$, TNF and IL-6, are elevated in OA [10] 


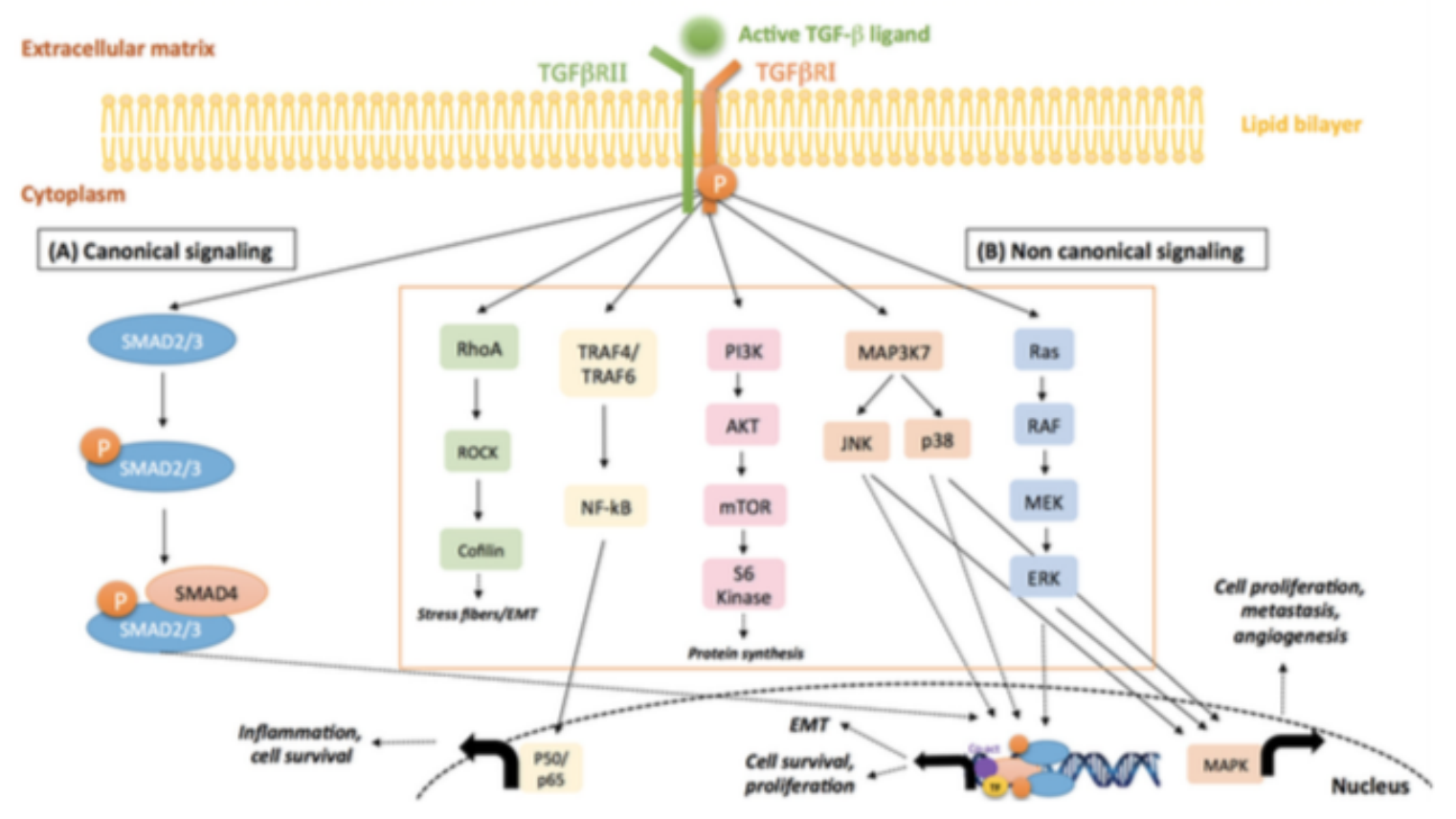

Figure 1.3 Canonical and non-canonical TGF- $\beta$ signaling pathways. (A) In the canonical signaling pathway, biologically active TGF- $\beta$ ligands bind to TGF $\beta$ RII, which in turn activates TGF $\beta$ RI. TGFßRI-regulated SMAD2/3 proteins are phosphorylated at their C-terminal serine residues and form complexes with SMAD4 (co-SMAD), initiating a number of biological processes through transcriptional regulation of target genes. (B) In the non-canonical signaling pathways, the TGF- $\beta$ receptor complex transmits its signal through other factors, such as the mitogen-activated protein kinases (MAPKs), phosphatidylinositide 3-kinase (PI3K), and TNF receptor-associated factor 4/6 (TRAF4/6). Activated MAPKs can exert transcriptional regulation either through direct interaction with the nuclear SMAD protein complex or via other downstream proteins. Moreover, TGF- $\beta$ activation of the TRAF proteins can initiate nuclear factor- $\kappa \mathrm{B}(\mathrm{NF}-\kappa \mathrm{B})$ signaling activity, leading to the inflammatory response among other processes [101]. 


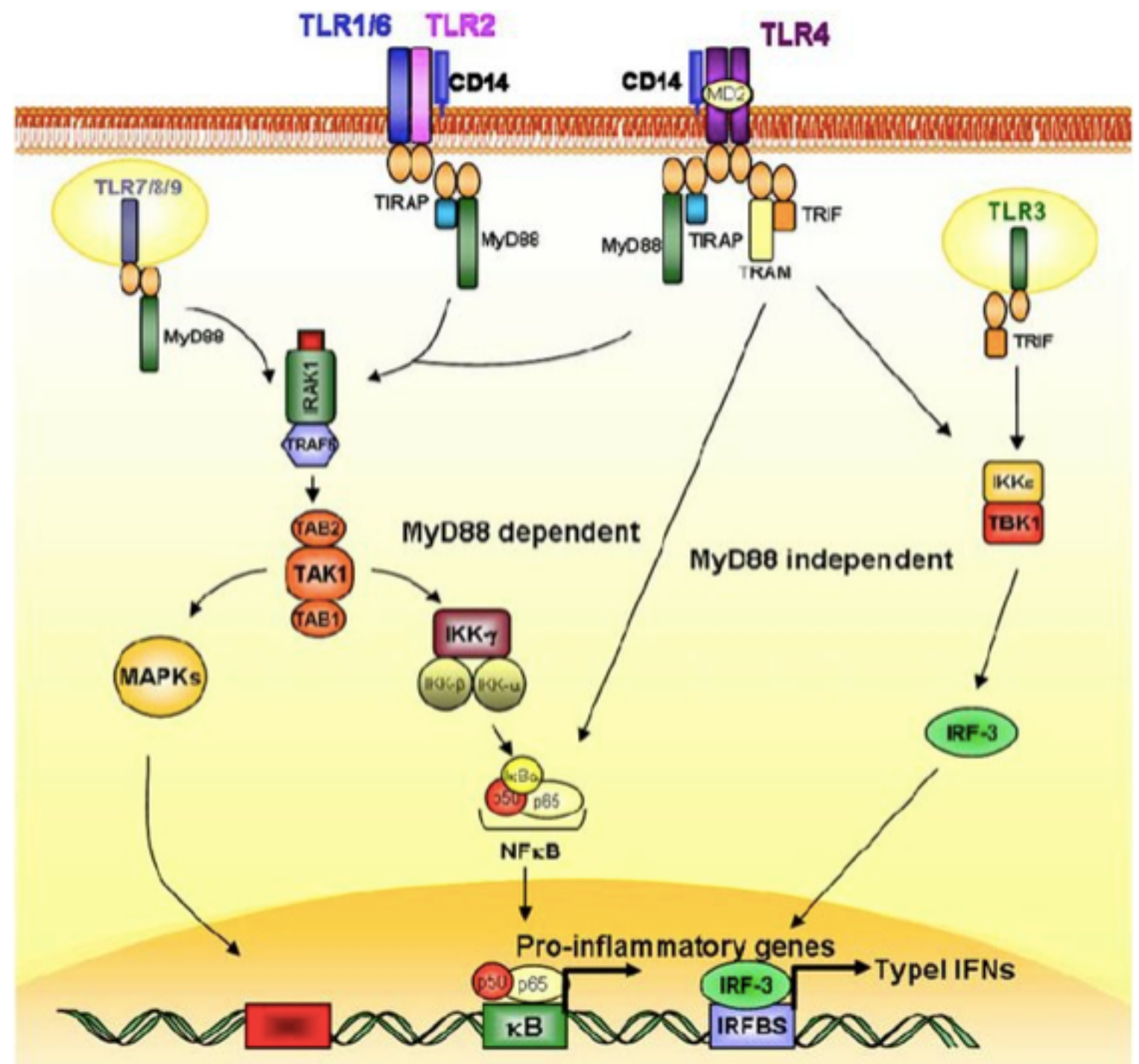

Figure 1.4 Toll-like receptor signaling pathway. Toll-like receptors (TLRs; with the exception of TLR3) induce nuclear factor- $\kappa$ B (NF- $\kappa$ B) dependent cytokine production through a pathway involving the adaptor molecule myeloid differentiation primary-response gene 88 (MyD88). However, TLR3 (and TLR4) transduce signals through a MyD88-independent signaling pathway that involves the adaptor molecule Toll/IL-1 receptor domain-containing adaptor inducing IFN- $\beta$ (TRIF) [102]. 


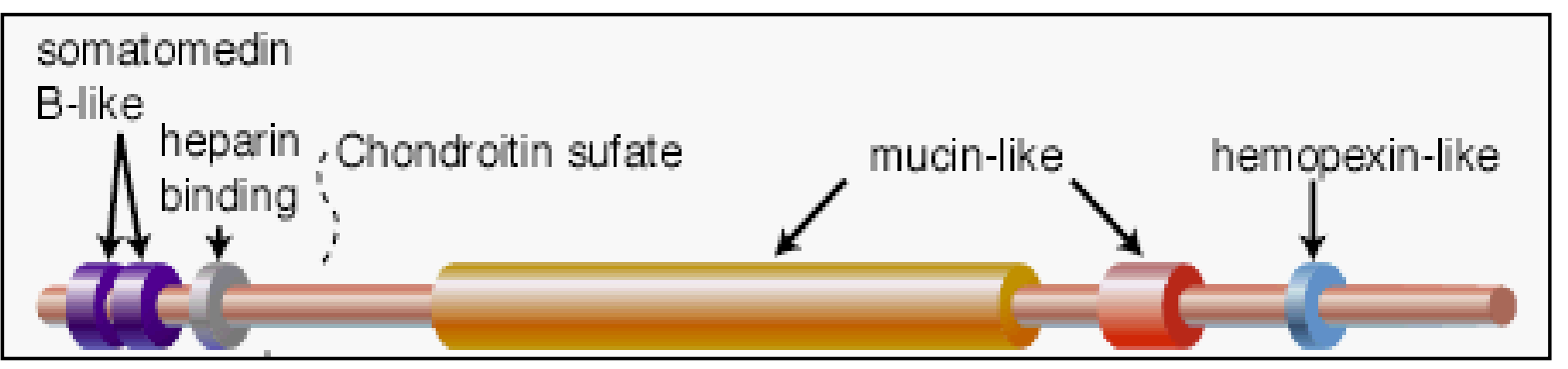

Figure 1.5 Schematic depicting the various motifs within the 1,404 amino acid proteoglycan4 (PRG4) polypeptide. 


\section{CHAPTER 2}

Role of CD44 in Regulating Toll-like Receptor 2 (TLR2) Activation of Human Macrophages and Downstream Expression of Proinflammatory Cytokines

Authors: Marwa Qadri, Sara Almadani, Gregory D. Jay and Khaled A. Elsaid

The following chapter was published in J Immunol December 1, 2017, ji1700713; DOI: https://doi.org/10.4049/jimmunol.1700713 


\section{Abstract}

Osteoarthritis (OA) is a low-grade chronic inflammatory joint disease. Innate immunity contributes to OA progression, mediated by toll-like receptors (TLR2 and TLR4). We evaluated the role of CD44, a transmembrane glycoprotein, in regulating TLR2-linked macrophage activation and resultant proinflammatory responses. TLR2 stimulation was performed on differentiated THP-1 macrophages in the presence or absence of a CD44-specific antibody or hyaluronan (HA). Nuclear factor kappa B (NFאB) nuclear translocation; interleukin-1 beta (IL$1 \beta)$ and tumor necrosis factor alpha $(\mathrm{TNF}-\alpha)$ gene expression and protein concentrations were determined. Anti-CD44 antibody and HA treatments reduced NFאB translocation, IL-1 $\beta$ and TNF- $\alpha$ expression and production $(p<0.001)$. Inhibition of proinflammatory response in macrophages by HA was mediated by CD44. Protein phosphatase 2A (PP2A) mediated the

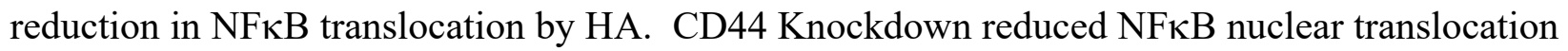
and downstream IL-1 $\beta$ and TNF- $\alpha$ protein production following TLR2 receptor stimulation $(p<0.001) . C D 44^{+/+}$murine bone marrow derived macrophages (BMDMs) produced higher TNF$\alpha$ compared to $C D 44^{-/}$macrophages following TLR2 stimulation $(p<0.01)$. HA dose-dependently inhibited TLR2 induced TNF- $\alpha$ production by murine BMDMs $(p<0.001)$. OA synovial fluids (SF) stimulated TLR2 and TLR4 receptors and induced NFאB translocation in THP-1 macrophages. Anti-CD44 antibody treatment significantly reduced macrophage activation by OA $\mathrm{SF}(p<0.01)$. CD44 regulated TLR2 responses in human macrophages, whereby a reduction in CD44 levels or engagement of CD44 by its ligand (HA) or a CD44-specific antibody reduced $\mathrm{NF \kappa B}$ translocation and downstream proinflammatory cytokine production. A CD44-specific 
antibody reduced macrophage activation by OA synovial fluids and CD44 is a potentially novel target in OA treatment. 


\subsection{Introduction}

Osteoarthritis (OA) is a degenerative joint disease involving joint structures e.g. the articular cartilage, synovium and subchondral bone $[103,104]$. Risk factors for OA pathogenesis include age, obesity, traumatic joint injury, and low-grade chronic inflammation [103, 105-109]. Inflammation of the synovial tissue is recognized as an active contributor to OA pathogenesis with multiple studies showing a correlation between the extent of synovitis and pain, cartilage erosion and disease progression [110-114]. The synovium is a soft tissue that is comprised of a surface layer, the intima and an underlying subintima $[112,115]$. The intima of normal synovium is 1-3 cell layers thick, with two cell types: fibroblast-like synoviocytes and macrophages $[115,116]$. Chronically inflamed synovium, as seen in OA, is characterized by synovial intimal hyperplasia, immune cell infiltration, subintimal fibrosis and neovascularization $[117,118]$.

Infiltrating immune cells detected most frequently in OA synovial tissues include macrophages, $\mathrm{T}$ cells and to a lesser extent mast cells and B cells $[117,119,120]$. Synovial macrophages play an important role in driving OA pathogenesis due to their significantly higher production of proinflammatory cytokines e.g. interleukin-1 beta (IL-1 $1 \beta$ ) and tumor necrosis factor alpha (TNF$\alpha$ ) compared to fibroblast-like synoviocytes [121, 122]. Activation of synovial macrophages was shown to promote synovial thickening, osteophyte formation and cartilage degeneration in experimental OA models $[123,124]$. As a component of the innate immune system, macrophages can be activated by damage-associated molecular patterns (DAMPs) through interaction with pattern recognition receptors, e.g. toll-like receptors (TLRs) on the surface of macrophages [125]. Examples of DAMPs in OA include extracellular matrix breakdown products e.g. biglycan, fibronectin fragments, low molecular weight hyaluronic acid, plasma proteins e.g. $\alpha 1$ and $\alpha 2$ 
macroglobulins, intracellular alarmins e.g. high mobility group box 1 (HMGB1) and crystals e.g. uric acid [126].

Hyaluronan (HA) is produced by fibroblast-like synoviocytes and is a major component of the synovial fluid. HA exerts important functions in the joint and its biological effects are mediated by its binding to its transmembrane receptor, cluster determinant 44 (CD44) [127]. In OA synoviocytes, HA suppresses IL-1 $\beta$ mediated nuclear factor kappa B (NFkB) activation and downstream expression of matrix metalloproteinases (MMPs) in a CD44-dependent manner [128, 129]. Additionally, HA's anti-inflammatory effects may be mediated by its ability to modulate TLR2 and TLR4 cartilage expression in experimental arthritis [130].

In this work, we aimed to evaluate the role of CD44 in regulating NFkB activation and proinflammatory cytokine production in response to TLR2 receptor activation in human macrophages, using a combination of CD44 receptor knockdown, TNF- $\alpha$ production by macrophages from CD44 wildtype and knockout mice, and CD44 receptor engagement by CD44 neutralizing antibody and $\mathrm{HA}$ treatments. We hypothesized that $\mathrm{CD} 44$ functions to regulate $\mathrm{NF} \kappa \mathrm{B}$ activation and downstream IL-1 $\beta$ and TNF- $\alpha$ gene expression and production in response to TLR2 stimulation of a human macrophage cell line. Finally, we studied TLR2 and TLR4 receptor activation by OA synovial fluids and examined the effect of CD44 targeting in suppressing the activation of macrophages by these fluids.

\subsection{Materials and Methods}

\subsubsection{Differentiation of THP-1 monocytes into macrophages}

THP-1 monocytes were obtained from American Type Culture Collection (ATCC, USA). Cells were cultured to a density of $1.5 \times 10^{6}$ cells $/ \mathrm{mL}$ in $75 \mathrm{~cm}$ flask in RPMI 1640 medium 
supplemented with $10 \%$ heat-inactivated fetal bovine serum (FBS), 10mM HEPES, 2mM glutamine, $100 \mathrm{U} / \mathrm{L}$ penicillin and $100 \mu \mathrm{g} / \mathrm{mL}$ streptomycin and maintained at $37^{\circ} \mathrm{C}$ under $5 \% \mathrm{CO}_{2}$. In sterile 12 well plates (Corning, Sigma Aldrich, USA), 600,000 cells in 2 ml RPMI 1640 media were differentiated into macrophages by incubation with phorbol 12-myristate-13-acetate (PMA; Sigma Aldrich) to a final concentration of $5 \mathrm{ng} / \mathrm{ml}$ for 48 hours [131]. Subsequently, media supernatants were removed and wells were washed three times with sterile PBS to remove unattached cells and new RPMI 1640 media was added.

\subsubsection{CD44, TLR2 and TLR4 expression in THP-1 macrophages}

THP-1 macrophages were incubated with CD44-specific, TLR2-specific or TLR4-specific antibody (Abcam, USA) at a final concentration of $1 \mu \mathrm{g}$ per 600,000 cells for 1 hour at $4{ }^{\circ} \mathrm{C}$. Cells were subsequently pelleted and the cell pellet was washed three times with PBS. THP-1 cells were subsequently incubated with DyLight ${ }^{\circledR} 488$ goat anti-mouse IgG (Abcam) at 1:500 dilution for 30 min at $4{ }^{\circ} \mathrm{C}$. Following cell pelleting and washing, $500 \mu \mathrm{L}$ of $4 \%$ paraformaldehyde was added and cell-associated fluorescence was determined by flow cytometry using BD FACSVerse (BD Biosciences, USA).

\subsubsection{Impact of TLR2 and TLR4 receptor activation on IL-1 $\beta$ and TNF- $\alpha$ production by THP-1 macrophages}

THP-1 macrophages (600,000 cells per well) were treated with Pam3CSK4 (Pam; Invivogen, USA), a TLR2 ligand [132], at a concentration range between 0.1 and $10 \mathrm{ng} / \mathrm{ml}$ for 24 hours. Similarly, THP-1 macrophages $(600,000$ cells per well) were treated with lipopolysaccharide (LPS; Invivogen, USA), a TLR4 ligand, at a concentration range between 0.1 and $10 \mathrm{ng} / \mathrm{ml}$ for 24 hours. Media supernatants were collected and IL- $1 \beta$ and TNF- $\alpha$ concentrations were determined using commercially available ELISA kits (R\&D systems, USA). 


\subsubsection{HA binding to recombinant human CD44 and TLR2 receptors}

High-binding microtiter plates (Corning, Sigma Aldrich) were used to coat recombinant human CD44 Fc chimeric protein or recombinant human TLR2 (R\&D systems) overnight at $4^{\circ} \mathrm{C}$. Each well received $100 \mu \mathrm{L}$ of CD44 or TLR2 protein $(1 \mathrm{ng} / \mathrm{ml}$ each) in PBS. Wells that were coated with CD44 or TLR2 were blocked with $2 \%$ bovine serum albumin (BSA; $300 \mu \mathrm{L}$ per well) for 2 hours at room temperature. Biotinylated HA (MW 1,500 kDa, Creative PEGWorks, USA) was added to the plate in serial dilutions and incubated for 1 hour at room temperature. Following washing with PBS $+0.1 \%$ Tween 20 , streptavidin-HRP (R\&D systems) was added at 1:10,000 dilution and incubated for 1 hour at room temperature. Following washing with PBS $+0.1 \%$ tween 20, the assay was developed using 1-step Turbo TMB ELISA reagent (Lifetechnologies, USA) and the absorbance was measured at $450 \mathrm{~nm}$. Data is presented as percent maximal binding. Data represents the mean \pm standard deviation (S.D.) of three independent experiments with duplicate wells per group.

\subsubsection{Impact of HA and CD44-specific antibody treatments on TLR2 ligand induced nuclear factor kappa B (NFkB) nuclear translocation in THP-1 monocytes and macrophages}

THP1-XBlue cells (Invivogen, USA) is a THP-1 monocyte cell line that stably expresses AP-1 and NFאB inducible secreted embryonic alkaline phosphatase (SEAP) reporter gene. Upon activation of TLR2, SEAP is secreted and its activity can be monitored using an alkaline phosphatase substrate [133]. THP1-XBlue cells were cultured in RPMI 1640 media supplemented with $4.5 \mathrm{~g} / \mathrm{L}$ glucose, $10 \% \mathrm{FBS}, 50 \mathrm{U} / \mathrm{mL}$ penicillin, $50 \mu \mathrm{g} / \mathrm{mL}$ streptomycin, $100 \mu \mathrm{g} / \mathrm{mL}$ normocin and $2 \mathrm{mM}$ L-glutamine.

THP1-XBlue cells (50,000 cells per well in HEK detection media) (100 $\mu \mathrm{L}$ per well) were treated with Pam $(5 \mathrm{ng} / \mathrm{ml})$ in the absence or presence of HA (MW $>950 \mathrm{kDa}$; R\&D systems) at a final 
concentration of 250 and $500 \mu \mathrm{g} / \mathrm{ml}$, an anti-CD44 antibody $(2.5 \mu \mathrm{g} / \mathrm{ml}$; Abcam) or an isotype control antibody ( $2.5 \mu \mathrm{g} / \mathrm{ml}$; Abcam) for 24 hours followed by measuring the $630 \mathrm{~nm}$ absorbance.

THP-1 macrophages (600,000 cells per well) were treated with Pam $(5 \mathrm{ng} / \mathrm{ml})$ in the absence or presence of HA (100, 250 and $500 \mu \mathrm{g} / \mathrm{ml})$ for 1 hour followed by cell harvesting and nuclear protein extraction using a commercially available kit (Thermo Fisher Scientific, USA). Protein content in the nuclear extract was quantified in all experimental groups and a total of $3 \mu \mathrm{g}$ of total protein was used to quantify NFkB p65 subunit nuclear levels using a DNA binding ELISA assay (Abcam). In another set of experiments, THP-1 macrophages were pre-treated with okadaic acid ( $5 \mathrm{nM}$; Tocris Biosciences, USA), a potent inhibitor of protein phosphatase $2 \mathrm{~A}$, for 2 hours followed by treatment with Pam $(5 \mathrm{ng} / \mathrm{ml})$ in the absence or presence of HA $(250 \mu \mathrm{g} / \mathrm{ml})$ for 1 hour followed by nuclear NFאB p65 subunit quantification as described above. Data is presented as detectable nuclear p65 levels normalized to control. Data represents the mean \pm S.D. of three independent experiments with duplicate wells per treatment.

\subsubsection{Impact of HA and CD44-specific antibody treatments on TLR2 ligand induced IL-1 $\beta$ and TNF- $\alpha$ gene expression and production in THP-1 macrophages}

THP-1 macrophages $(600,000$ cells per well) were treated with Pam $(1 \mathrm{ng} / \mathrm{ml})$ in the absence or presence of HA $(100,500$ and $1,000 \mu \mathrm{g} / \mathrm{ml})$ for 6 hours followed by RNA extraction using TRIzol reagent (Thermo Fisher Scientific) and RNA concentrations were determined using a NanoDrop ND-2000 spectrophotometer (NanoDrop Technologies, USA). cDNA was synthesized using Transcriptor First Strand cDNA Synthesis Kit (Roche, USA). Quantitative PCR (qPCR) was performed on Applied Biosystems StepOnePlus Real-Time PCR System (Thermo Fisher Scientific) using TaqMan Fast Advanced Master Mix (Life Technologies). The cycle threshold (Ct) value of IL-1 $\beta$ (Hs00174097_m1; Thermo Fisher Scientific) was normalized to the Ct value 
of GAPDH (Hs02758991_g1; Thermo Fisher Scientific) in the same sample, and the relative expression was calculated using the $2^{-\Delta \Delta C t}$ method [134]. Data is presented as fold target gene expression compared to untreated control.

In another set of experiments, THP-1 macrophages (600,000 cells per well) were treated with Pam (5ng/ml) in the absence or presence of HA $(100,500$ and $1,000 \mu \mathrm{g} / \mathrm{ml})$, anti-CD44 antibody $(2.5$ $\mu \mathrm{g} / \mathrm{ml})$ or isotype control antibody $(2.5 \mu \mathrm{g} / \mathrm{ml})$ for 24 hours. IL-1 $\beta$ and TNF- $\alpha$ media concentrations were determined using commercially available ELISA kits.

\subsubsection{Impact of HA treatment on TLR2 ligand induced CD44 expression in THP-1 macrophages}

THP-1 macrophages (600,000 cells per well) were treated with Pam $(5 \mathrm{ng} / \mathrm{ml})$ in the absence or presence of HA $(100,500$ and $1,000 \mu \mathrm{g} / \mathrm{ml})$ for 6 hours followed by RNA isolation, cDNA synthesis and qPCR as described above. The Ct value of CD44 (Hs01075864_m1; Thermo Fisher Scientific) was normalized to the Ct value of GAPDH in the same sample, and the relative expression was determined as described above. Data is presented as fold CD44 expression compared to untreated control.

In another set of experiments, THP-1 macrophages were treated as described above for 24 hours followed by cell harvest. THP-1 macrophages were incubated with anti-CD44 antibody ( $1 \mu \mathrm{g}$ per 600,000 cells) for 1 hour at $4^{\circ} \mathrm{C}$. Cells were subsequently pelleted and the cell pellet was washed three times with PBS. THP-1 cells were subsequently incubated with DyLight ${ }^{\circledR} 488$ goat antimouse $\operatorname{IgG}$ at 1:500 dilution for $30 \mathrm{~min}$ at $4^{\circ} \mathrm{C}$. Following cell pelleting and washing, cellassociated fluorescence was determined as described above. 


\subsubsection{CD44 receptor knockdown and associated TLR2 ligand induced IL-1 $\beta$ and TNF- $\alpha$ production in THP-1 macrophages and role of HA}

THP-1 macrophages $(600,000$ cells per well) were treated with a pre-validated CD44 small interfering RNA (siRNA) (Thermo Fisher Scientific) (25 pmoles per well) or a non-targeted negative control (NC) siRNA (25pmoles) (Thermo Fisher Scientific) for 48 hours. Transfection was performed using Lipofectamine RNAiMax (Thermo Fisher Scientific) per manufacturer's recommendations. To confirm CD44 knockdown, CD44 gene expression was determined in CD44 siRNA and NC siRNA-treated THP-1 macrophages as described above and compared to CD44 expression in untreated control macrophages. Additionally, CD44 and TLR2 protein levels in CD44 siRNA, NC siRNA-treated and untreated control THP-1 macrophages were determined using flow cytometry as described above.

NFkB p65 subunit nuclear levels in CD44 siRNA, NC siRNA-treated and untreated control THP1 macrophages $(600,000$ cells per well) following treatment with Pam $(5 \mathrm{ng} / \mathrm{ml})$ for 1 hour were determined as described above. CD44 siRNA, NC siRNA-treated and untreated control THP-1 macrophages $(600,000$ cells per well) were treated with Pam $(5 \mathrm{ng} / \mathrm{ml})$ for 24 hours. In another set of experiments, Pam treatment was performed in the absence or presence of $\mathrm{HA}(500 \mu \mathrm{g} / \mathrm{mL})$. IL$1 \beta$ and TNF- $\alpha$ media concentrations were determined as described above.

\subsubsection{TLR2 ligand induced TNF- $\alpha$ production in murine bone marrow derived macrophages from $C D 44^{+/+}$and $C D 44^{-/}$mice and effect of HA treatment on primary murine macrophages}

$C D 44^{-/}\left(\mathrm{JAX}\right.$ stock \# 005085) and $C D 44^{+/+}(\mathrm{JAX}$ stock \# 00664) pathogen-free male mice $(\mathrm{n}=8$ in each group) were acquired from the Jackson Laboratory (Maine, USA) [135]. Animals (10-14 weeks old) were euthanized and isolation and culture of bone marrow derived macrophages (BMDMs) was performed as previously described [136]. Both femurs and tibia bones were carefully dissected and bone marrows were flushed using a sterile $25 \mathrm{G}$ needle filled with 
DMEM/F12 medium (Fisher Scientific) supplemented with 10ng/ml macrophage colonystimulating factor (M-CSF; R\&D systems). Cells were subsequently cultured for 7 days with media change on day 3. On day 7, BMDMs were gently scraped and dislodged using Corning Cellstripper Solution (VWR, USA) for $5 \mathrm{~min}$ at $37^{\circ} \mathrm{C}$. An equal volume of macrophage complete medium was added to the cells and the cells were centrifuged for $10 \mathrm{~min}$ at $400 \mathrm{xg}$ and $4^{\circ} \mathrm{C}$, and the supernatant was discarded. Subsequently, BMDMs were plated to perform TLR2 stimulation studies or to characterize surface markers $\mathrm{Cd} 11 \mathrm{~b}$ and F4/80 expression using flow cytometry.

BMDMs $(400,000$ cells) were treated with FITC-labeled anti-CD11b antibody (abcam) (1:500 dilution), PE-Texas Red-labeled anti- F4/80 antibody (ThermoFisher Scientific) (1:500 dilution) or FITC-labeled Rat IgG2b, kappa monoclonal - Isotype control (Abcam) for $1 \mathrm{hr}$ at $37^{\circ} \mathrm{C}$. Cells were subsequently pelleted and the cell pellet was washed three times with PBS. Following cell pelleting and washing, $500 \mu \mathrm{L}$ of $4 \%$ paraformaldehyde were added and cell-associated fluorescence was determined by flow cytometry.

BMDMs from both genotypes were plated overnight in 12 well plates $(200,000$ cells per well). Cells were treated with Pam $(1 \mathrm{ng} / \mathrm{ml}$ or $5 \mathrm{ng} / \mathrm{ml})$ for 6 hours at $37^{\circ} \mathrm{C}$ and TNF- $\alpha$ media concentrations were determined using a commercially available ELISA (R\&D Systems). In another set of experiments, $C D 44^{+/+}$BMDMs were treated with Pam $(5 \mathrm{ng} / \mathrm{ml})$ in the absence or presence of HA $(100,250$ and $500 \mu \mathrm{g} / \mathrm{ml})$ for 6 hours at $37^{\circ} \mathrm{C}$ and TNF- $\alpha$ media concentrations were determined as above. Data is presented as the mean TNF- $\alpha$ concentrations \pm S.D. of 4 independent experiments, with triplicate wells per treatment. All animal tissue harvests were approved by the IACUC committee at Chapman University. 


\subsubsection{Activation of TLR2 and TLR4 receptors by synovial fluids from patients with OA and the role of CD44 in regulating macrophage activation in response to OA synovial fluids}

Synovial fluid aspirates (SF) were collected from patients with OA ( $\mathrm{n}=12)$ (Articular Engineering, USA) following knee replacement surgery or from donors within 24 hours of death, collected with partner site IRB approval with informed written consent from the donor or nearest relative. A total of 6 patients were female. The median age (interquartile range) of the group was 71 (60 to 82). Normal SF specimens $(n=4)$ were obtained from subjects with no clinical history of joint disease or arthritis, and were provided by Dr. Martin Lotz from the Scripps Research Institute, USA. Screening of OA SF specimens for activation of TLR2 and TLR4 receptors was performed by incubation with TLR2-HEK and TLR4-HEK cells (25,000 cells per well in HEK Blue detection media) (Invivogen). The volume of SF aspirates was $7.5 \mu \mathrm{L}$ per well, corresponding to $3.75 \%$ dilution. The final volume in each well was $200 \mu \mathrm{L}$. Activation of TLR2 or TLR4 in these cells results in nuclear translocation of $\mathrm{NF \kappa B}$ and expression of SEAP, whose activity can be detected in the culture media. Cells were incubated with the SF samples for 48 hours at $37^{\circ} \mathrm{C}$. The $630 \mathrm{~nm}$ absorbance was measured and normalized to the $630 \mathrm{~nm}$ absorbance values of untreated control cells.

THP1-XBlue monocytes (25,000 cells per well) were maintained in serum-free RPMI 1640 for 48 hours followed by differentiation of the THP-1 monocytes into macrophages as described above. SF aspirates with detectable TLR2 and TLR 4 activity were incubated with THP1-XBlue macrophages for 48 hours in HEK Blue detection media in the absence or presence a CD44specific antibody $(2.5 \mu \mathrm{g} / \mathrm{ml})$, TLR2-specific antibody (Abcam; $2.5 \mu \mathrm{g} / \mathrm{ml})$ or isotype control $(2.5$ $\mu \mathrm{g} / \mathrm{ml}$ ). Normal SF specimens were used as controls. The $630 \mathrm{~nm}$ absorbance was subsequently measured. Data is presented as the mean $630 \mathrm{~nm}$ absorbance value of each SF specimen, based on two independent experiments with duplicate wells per experiment. 


\subsubsection{Statistical Analyses}

Unless otherwise specified, data is presented as the mean \pm S.D. of 4 independent experiments with at least duplicate wells per group. Continuous variables were tested for normality and equal variances. Variables that satisfied both assumptions were tested for statistical significance using Student's $t$-test for two group comparisons. Multiple group comparisons were performed using analysis of variance with Tukey's post-hoc test. Variables that did not satisfy the normality assumption were tested using Mann-Whitney U test or ANOVA on the ranks. The level of statistical significance was $a$ priori set at $\alpha=0.05$.

\subsection{Results}

\subsubsection{CD44 and TLR receptor expression in human THP-1 macrophages and proinflammatory cytokine production}

A representative flow cytometry histogram depicting cell-associated fluorescence of THP-1 macrophages following probing with CD44, TLR2 or TLR4 antibodies is shown in figure 2.1A. Compared to unstained cells, cell associated fluorescence for CD44 and TLR2 exhibited a right shift indicating expression of CD44 and TLR2 by THP-1 macrophages. On the contrary, cell associated fluorescence for TLR4 was not qualitatively different from unstained control cells. TLR2 receptor activation resulted in a concentration-dependent elevation in media IL-1 $\beta$ (fig. 2.1B) and TNF- $\alpha$ (fig. 2.1C) concentrations. Similarly, TLR4 receptor activation resulted in detectable IL-1 $\beta$ and TNF- $\alpha$ levels. Across all ligand treatments, TLR2 activation resulted in a significantly higher $(p<0.001)$ IL-1 $\beta$ and TNF- $\alpha$ production compared to TLR4 activation. 


\subsubsection{HA and CD44-specific antibody treatments reduced TLR2 ligand induced NFKB nuclear translocation in THP-1 monocytes and macrophages}

HA binding to immobilized TLR2 and CD44 receptors is shown in figure 2.2A. HA exhibited concentration-dependent binding to CD44 with no detectable binding to TLR2. Pam treatment resulted in NFאB nuclear translocation in THP-1 monocytes compared to untreated cells $(p<0.001)$ (fig. 2.2B). HA ( 250 and $500 \mu \mathrm{g} / \mathrm{mL}$ ) treatment reduced NFкB nuclear translocation in THP-1 monocytes $(p<0.01 ; p<0.001)$ following TLR2 receptor activation. Similarly, Anti-CD44 antibody treatment reduced NFkB nuclear translocation in THP-1 monocytes $(p<0.001)$. In contrast, isotype control antibody treatment did not alter NFkB nuclear translocation. There was no difference in the magnitude of reduction of NFkB nuclear translocation in THP-1 monocytes with HA ( $500 \mu \mathrm{g} / \mathrm{mL})$ or a CD44 antibody treatments.

TLR2 receptor activation resulted in NFKB p65 subunit nuclear translocation in THP-1 macrophages compared to untreated cells $(p<0.001)$ (fig. $2.2 \mathrm{C})$. HA $(250$ and $500 \mu \mathrm{g} / \mathrm{mL})$ treatments reduced p65 subunit translocation $(p<0.01)$ in THP-1 macrophages following TLR2 receptor activation. Okadaic acid treatment did not alter NFאB p65 subunit translocation following TLR2 receptor activation (fig. 2.2D). NFKB p65 subunit nuclear levels were significantly higher in the Pam $+\mathrm{HA}+$ Okadiac acid group compared to Pam + HA group $(p<0.01)$.

\subsubsection{HA and CD44-specific antibody treatments reduced TLR2 ligand stimulated proinflammatory cytokine expression and production in THP-1 macrophages}

TLR2 activation induced IL-1 $\beta$ gene expression and production in THP-1 macrophages $(p<0.001)$

(fig. 2.3A and fig. 2.3C). HA (500 and $1,000 \mu \mathrm{g} / \mathrm{mL})$ treatment reduced IL-1 $\beta$ gene expression in THP-1 macrophages following TLR2 activation $(p<0.05 ; p<0.01)$ (fig. 2.3A). Correspondingly, HA (500 and $1,000 \mu \mathrm{g} / \mathrm{mL})$ treatment reduced IL-1 $\beta$ production by THP-1 macrophages $(p<0.001)$ (fig. 2.3C). TLR2 activation induced TNF- $\alpha$ gene expression and production in THP-1 
macrophages ( $p<0.001$ ) (fig. 2.3B and fig. 2.3D). HA (500 and 1,000 $\mu \mathrm{g} / \mathrm{mL}$ ) treatment reduced TNF- $\alpha$ gene expression in THP-1 macrophages following TLR2 activation $(p<0.01)$ (fig. 2.3B). Similarly, HA (500 and $1,000 \mu \mathrm{g} / \mathrm{mL}$ ) treatment reduced TNF- $\alpha$ production by THP-1 macrophages $(p<0.001)$ (fig. 2.3D). CD44 antibody treatment reduced IL-1 $\beta$ and TNF- $\alpha$ media supernatant concentrations following TLR2 activation ( $p<0.001)$ (Fig. 2.3E and fig. 2.3F). In contrast, IL- $1 \beta$ and TNF- $\alpha$ media concentrations were not significantly different between Pam alone and Pam + isotype control (IC) antibody groups.

\subsubsection{HA reduced CD44 expression following TLR2 receptor activation in THP-1 macrophages}

TLR2 activation induced CD44 gene expression in THP-1 macrophages $(p<0.001)$ (fig. 2.4A). HA $(100,500$ and $1,000 \mu \mathrm{g} / \mathrm{mL})$ treatment reduced CD44 expression in THP-1 macrophages following TLR2 activation $(p<0.01)$. A dose-response for HA treatment was observed as CD44 expression in the Pam + HA $(1,000 \mu \mathrm{g} / \mathrm{mL})$ group was significantly lower than CD44 gene expression in the Pam $+\mathrm{HA}(500 \mu \mathrm{g} / \mathrm{mL})$ and Pam $+\mathrm{HA}(100 \mu \mathrm{g} / \mathrm{mL})(p<0.01 ; p<0.001)$. A representative flow cytometry histogram depicting cell-associated fluorescence of THP-1 macrophages following probing with CD44 antibody is presented in figure 2.4B. TLR2 activation resulted in a right shift of the cell population indicating increased CD44 protein levels on macrophages. HA treatments showed a qualitative reduction in mean cell associated fluorescence, indicating a reduction in CD44 receptor levels on macrophages. Semi-quantitative analysis of mean cell-associated fluorescence is shown in figure 2.4C. Mean fluorescence in the Pam alone group was significantly higher compared to untreated control group $(p<0.001)$. Mean cellassociated fluorescence in the Pam $+\mathrm{HA}(1,000 \mu \mathrm{g} / \mathrm{mL})$ and Pam $+\mathrm{HA}(500 \mu \mathrm{g} / \mathrm{mL})$ were significantly lower than in the Pam group $(p<0.001)$. Similarly, mean cell-associated fluorescence 
in the Pam + HA $(1,000 \mu \mathrm{g} / \mathrm{mL})$ and Pam + HA $(500 \mu \mathrm{g} / \mathrm{mL})$ were significantly lower than in the Pam + HA $(100 \mu \mathrm{g} / \mathrm{mL})$ group $(p<0.01)$.

\subsubsection{Role of CD44 in regulating downstream responses of TLR2 activation in THP-1 macrophages}

The impact of CD44 silencing on CD44 gene expression and protein is shown in figures $2.5 \mathrm{~A}$ and 2.5B. CD44 silencing was achieved with approximately $85 \%$ reduction in CD44 gene expression and $60 \%$ reduction in CD44 protein. CD44 knockdown did not alter TLR2 expression in THP-1 macrophages (fig. 2.5C). CD44 knockdown resulted in a significant reduction in NFkB p65 subunit nuclear levels following TLR2 activation compared to NC siRNA-treated or control THP1 macrophages $(p<0.001)$ (fig. 2.5D). CD44 knockdown resulted in a significant reduction in IL$1 \beta$ and TNF- $\alpha$ production following TLR2 activation compared to NC siRNA-treated or control THP-1 macrophages ( $p<0.001$ ) (fig. 2.5E and 2.5F). There was no significant difference in Pamstimulated IL-1 $\beta$ or TNF- $\alpha$ media concentrations between NC siRNA-treated and control THP-1 macrophages. HA $(500 \mu \mathrm{g} / \mathrm{mL})$ treatment reduced IL- $1 \beta$ and TNF- $\alpha$ media concentrations following TLR2 receptor activation in NC siRNA treated and control THP-1 macrophages $(p<0.001)$ (fig. 2.6A and 2.6B). In contrast, $\mathrm{HA}(500 \mu \mathrm{g} / \mathrm{mL})$ treatment did not significantly alter IL-1 $\beta$ and TNF- $\alpha$ media concentrations following TLR2 receptor activation in CD44 siRNAtreated THP-1 macrophages.

\subsubsection{CD44 modulated TLR2 ligand-induced TNF- $\alpha$ production by murine BMDMs and the HA treatment suppressed TLR2 ligand activation of primary murine BMDMs.}

TNF- $\alpha$ production by murine BMDMs from $C D 44^{-/}$and $C D 44^{+/+}$mice in response to TLR2 receptor activation and the impact of HA treatment is shown in figure 2.7. A representative flow cytometry scatterplot showing $\mathrm{CD} 11 \mathrm{~b}$ and $\mathrm{F} 4 / 80$ probing of murine BMDMs is presented in figure 2.7A. Murine BMDMs exhibited strong positive staining for both $\mathrm{CD} 11 \mathrm{~b}$ and F4/80 epitopes, 
with typically $90 \%$ or more of the cell population positive for both surface markers. Using twoway ANOVA, we identified a significant interaction between CD44 genotype and Pam concentrations ( $p=0.0035)$. TNF- $\alpha$ media concentrations in the Pam $(1 \mathrm{ng} / \mathrm{ml}$ and $5 \mathrm{ng} / \mathrm{ml})$ treated $C D 44^{+/+}$BMDMs were significantly higher compared to Pam-treated $C D 44^{-/}$BMDMs (fig. 2.7B) $(p<0.001 ; p<0.01)$. The mean TNF- $\alpha$ media concentration in the Pam $(1 \mathrm{ng} / \mathrm{ml})$ treated $C D 44^{-/}$ BMDMs group was approximately $67 \%$ lower than corresponding mean TNF- $\alpha$ media concentrations in the Pam-treated $C D 44^{+/+}$BMDMs. Similarly, the mean TNF- $\alpha$ media concentration in the Pam $(5 \mathrm{ng} / \mathrm{ml})$ treated $C D 44^{-/}$BMDMs group was approximately $62 \%$ lower than the corresponding mean TNF- $\alpha$ media concentration in the Pam-treated $C D 44^{+/+}$BMDMs. In $C D 44^{+/+}$BMDMs, TNF- $\alpha$ concentrations in the Pam ( 1 and $\left.5 \mathrm{ng} / \mathrm{ml}\right)$ groups were significantly higher than control group $(p<0.001)$. Additionally, TNF- $\alpha$ concentration in the Pam $(5 \mathrm{ng} / \mathrm{ml})$ group was significantly higher than the Pam $(1 \mathrm{ng} / \mathrm{ml})$ group $(p<0.001)$. In $C D 44^{-/}$BMDMs, TNF$\alpha$ concentrations in the Pam $(1 \mathrm{ng} / \mathrm{ml})$ group was not significantly different from control $C D 44^{-/}$ BMDMs $(\mathrm{p}=0.1207)$. Furthermore, TNF- $\alpha$ concentration in the Pam $(5 \mathrm{ng} / \mathrm{ml})$ group was significantly higher than the Pam $(1 \mathrm{ng} / \mathrm{ml})$ group $(p<0.001)$.

The impact of HA treatment on TLR2 ligand induced TNF- $\alpha$ production in $C D 44^{+/+}$murine BMDMs is shown in figure 2.7C. TNF- $\alpha$ media concentrations in the Pam $+\mathrm{HA}(100 \mu \mathrm{g} / \mathrm{ml})$ group were not significantly different from TNF- $\alpha$ media concentrations in the Pam alone group. TNF- $\alpha$ media concentrations in the Pam + HA $(250 \mu \mathrm{g} / \mathrm{ml})$ group were significantly lower than TNF- $\alpha$ media concentrations in the Pam alone and Pam + HA $(100 \mu \mathrm{g} / \mathrm{ml})$ groups $(p<0.001)$. Similarly, TNF- $\alpha$ media concentrations in the Pam + HA $(250 \mu \mathrm{g} / \mathrm{ml})$ group were significantly lower than TNF- $\alpha$ media concentrations in the Pam alone and Pam + HA $(100 \mu \mathrm{g} / \mathrm{ml})$ groups 
$(p<0.001)$. HA $(500 \mu \mathrm{g} / \mathrm{ml})$ alone treatment did not alter TNF- $\alpha$ production compared to untreated control cells.

\subsubsection{OA SF specimens activated TLR2 and TLR4 receptors and a CD44-specific antibody reduced activation of THP-1 macrophages by OA SF specimens.}

Activation of TLR2 and TLR4 receptors by OA SF specimens is shown in figure 2.8A. A total of 8 samples demonstrated significant activation of TLR2 and TLR4 receptors $(p<0.001)$. In contrast, 3 specimens did not exhibit activation of TLR2 or TLR4 and one sample activated TLR2 receptor but not the TLR4 receptor. OA SF specimens that activated TLR2 and TLR4 receptors also significantly stimulated $\mathrm{NF \kappa B}$ nuclear translocation in THP-1 macrophages compared to normal SF specimens $(p<0.001)$ (fig. 2.8B). TLR2 neutralizing antibody treatment significantly reduced OA SF activation of THP-1 human macrophages $(p<0.01)$. Similarly, CD44 antibody treatment significantly reduced OA SF activation of THP-1 human macrophages $(p<0.01)$.

\subsection{Discussion}

The TLR family comprises ten functional receptor subtypes with TLR 1-7 and 9 being detected in the synovial tissues of patients with OA $[107,118,137,138]$. A role for TLRs, specifically TLR2 and TLR4, in the pathogenesis of OA has been suggested [139, 140]. Progressive OA was associated with expression of TLR2 in cartilage and chondrocytes derived from a TLR2/TLR4 double knockout mouse showed attenuated matrix metalloproteinase-13 (MMP-13) expression in response to TLR2 stimulation [139, 140]. Furthermore, evidence of macrophage activation in the synovial lining was recently reported and the extent of synovial macrophage activation was shown to be associated with OA severity and joint pain [141]. In this work, we have examined macrophage activation in response to TLR2 and TLR4 activation. TLR2 and TLR4 ligands induced gene expression and production of IL-1 $\beta$ and TNF- $\alpha$ in a concentration-dependent 
manner. TLR2 activation produced higher levels of IL-1 $\beta$ and TNF- $\alpha$ compared to TLR4 across all ligand concentrations utilized. This may be due to the relative level of TLR2 and TLR4 expression on the surface of the macrophages. Using flow cytometry, we have detected increased TLR2 expression compared to TLR4. This finding is in line with other work that demonstrated enhanced TLR2 expression on THP-1 macrophages [142]. TLR2 activation resulted in NFkB nuclear translocation in a monocyte NFאB reporter assay. In macrophages, TLR2 ligands induced the nuclear translocation of NFאB p65 subunit. In addition to the induction of proinflammatory cytokines, TLR2 activation significantly increased CD44 gene expression and increased CD44 protein levels.

CD44 is a transmembrane receptor, with various isoforms generated by extensive alternative splicing and post-translational modifications [143]. In addition to its established role in mediating cell adhesion and migration, CD44 receptor has a role in regulating cell signaling pathways, by facilitating signaling protein recruitment and assembly [144]. In the context of innate immunity, CD44 was found to regulate Fc $\gamma$ and complement receptor 3-dependent macrophage phagocytosis [144]. Additionally, CD44 may play a role in the negative regulation of TLR receptor activation $[145,146]$. In this present study, a CD44 antibody treatment reduced NFאB nuclear translocation and downstream IL-1 $\beta$ and TNF- $\alpha$ gene expression and production. Additionally, reducing CD44 expression in human macrophages resulted in a significant attenuation of the latter's response to TLR2 receptor activation and downstream proinflammatory response. This attenuation is not related to the TLR receptor density on the surface of macrophages, as CD44 receptor knockdown did not modify TLR2 receptor density on THP-1 macrophages. The regulatory role of CD44 is further highlighted by a strongly attenuated proinflammatory response following TLR2 receptor 
stimulation of primary macrophages derived from CD44 knockout mice compared to macrophages derived from CD44 wildtype animals.

Protein phosphatase-2A (PP2A) is an abundant intracellular serine/threonine phosphatase with key roles in the regulation of many cellular functions including cellular proliferation and immune responses [147, 148]. Inhibition of PP2A by okadaic acid resulted in increased nuclear translocation of NFאB and AP-1 and IL-1 $\beta$ expression in THP-1 macrophages [147]. Additionally, CD44 engagement was shown to increase intracellular PP2A [149]. In our work, HA suppressed NFאB p65 subunit nuclear translocation in response to TLR2 activation, and that effect was shown to be CD44 dependent as HA failed to demonstrate an anti-inflammatory effect in response to TLR2 activation in macrophages following CD44 knockdown. The suppressive effect of HA was confirmed in primary murine macrophages as HA treatment dose-dependently reduced TNF- $\alpha$ production subsequent to TLR2 receptor stimulation. Furthermore, the effect of HA was mediate by intracellular PP2A activity, as inhibition of PP2A activity reduced the inhibitory effect of HA on NFkB activation. The observed anti-inflammatory activity of HA is not due to a direct interaction between HA and the TLR2 receptor as we did not observe any significant binding of HA to recombinant TLR2 receptor. The biological effect of HA in reducing TLR2 mediated proinflammatory response in macrophages is physiologically relevant. The concentration of HA in normal SF can vary between 2 and $4 \mathrm{mg} / \mathrm{ml}$ [150]. In OA SF aspirates, the concentration and molecular weight distribution of HA are significantly reduced, and this reduction was associated with TLR2 and TLR4 activation by OA SF aspirates $[150,151]$. This association may argue for an endogenous homeostatic role for HA in inhibiting synovial macrophage activation in response to TLR receptor stimulation by cartilage matrix degradation products. 
A majority of the aspirated SF samples from patients with advanced OA that we examined activated TLR2 and TLR4 receptors. This observation is in agreement with previous reports demonstrating activation of TLR2 and TLR4 receptors by OA SF, and augmentation of TLRmediated responses in OA fibroblast-like synoviocytes by SF from patients with early stage OA [151-153]. Interestingly, SF specimens that activated TLR2 and TLR4 receptors induced NFKB nuclear translocation in macrophages, while normal SF specimens treatments failed to activate macrophages. Macrophage TLR2 receptors appeared to mediate NFKB nuclear activation as neutralization of TLR2 abrogated macrophage activation by these SF specimens. CD44 antibody treatment produced a similar effect to TLR2 neutralization, providing further support to the utility of targeting CD44 to suppress macrophage response to DAMPs present in OA SF.

We have demonstrated that CD44 receptor plays a significant role in suppressing TLR2-linked $\mathrm{NF \kappa B}$ nuclear translocation and resultant proinflammatory response in macrophages, in a mechanism that involves intracellular PP2A. Neutralization of the CD44 receptor by its ligand hyaluronan, that binds to and is internalized by different cell types including macrophages [127], or by a monoclonal antibody resulted in inhibition of NFKB nuclear translocation. Absence of the CD44 receptor or its knockdown produced a similar effect to receptor neutralization by hyaluronan or an antibody. OA SF activated TLR2 and TLR4 receptors and correspondingly induced NFKB nuclear translocation in a mechanism that involves TLR2. Otherwise, a CD44-specific antibody reduced macrophage activation by these SF specimens. In conclusion, CD44 is a potentially novel target that may act to limit synovial macrophage activation by cartilage matrix degradation products in the joint.

This study expands the role of CD44 in OA pathogenesis. CD44 can bind different types of ligands and in addition to HA, CD44 was shown to bind proteoglycan-4 (PRG4), a major component of 
synovial fluids [154]. CD44 expression was shown to be associated with enhanced proliferation of synoviocytes from patients with OA, rheumatoid arthritis (RA) as well synoviocytes from Prg41- mice. Targeting the CD44 receptor by HA or by the recombinant form of PRG4 reduced cytokine-induced OA and RA synoviocyte proliferation. The regulatory role of CD44 in controlling downstream effects of IL-1 $\beta$ was further confirmed by the ability of PRG4 to inhibit

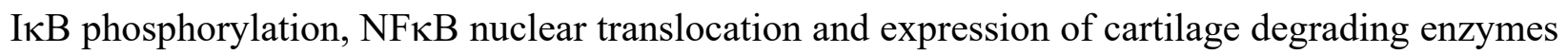
by OA synoviocytes in a CD44-dependent mechanism [155]. Our findings are limited by the low number of SF aspirates that we have investigated. Furthermore, we did not ascertain the molecular identity of the DAMPs in the SF aspirates that activated TLR receptors and induced NFkB nuclear translocation in THP-1 macrophages.

\subsection{Acknowledgements}

The authors would like to thank Dr. Martin Lotz for providing normal synovial fluids. 


\subsection{Figures}

A

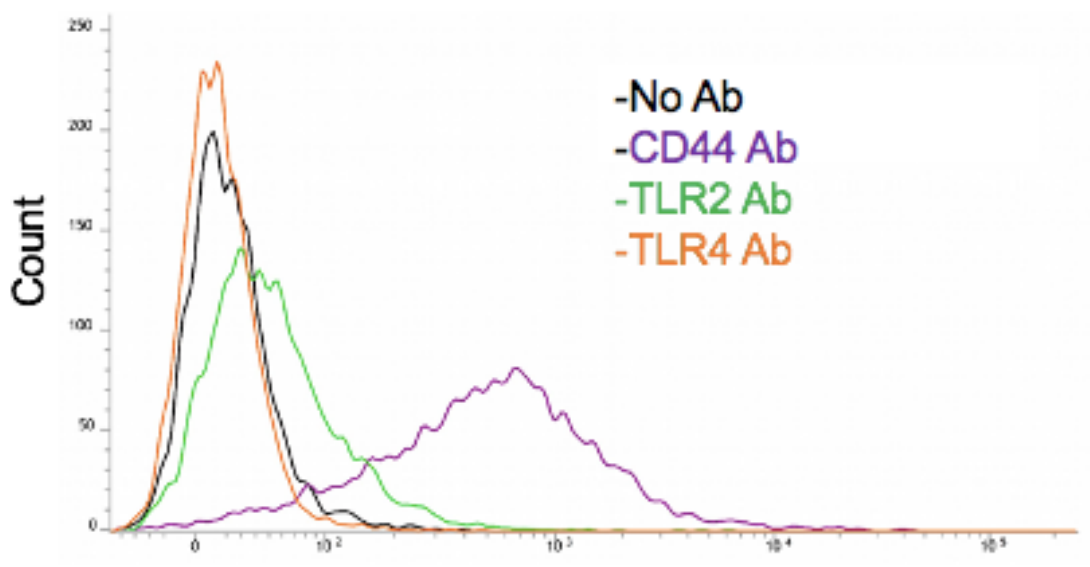

Fluorescence Intensity

B

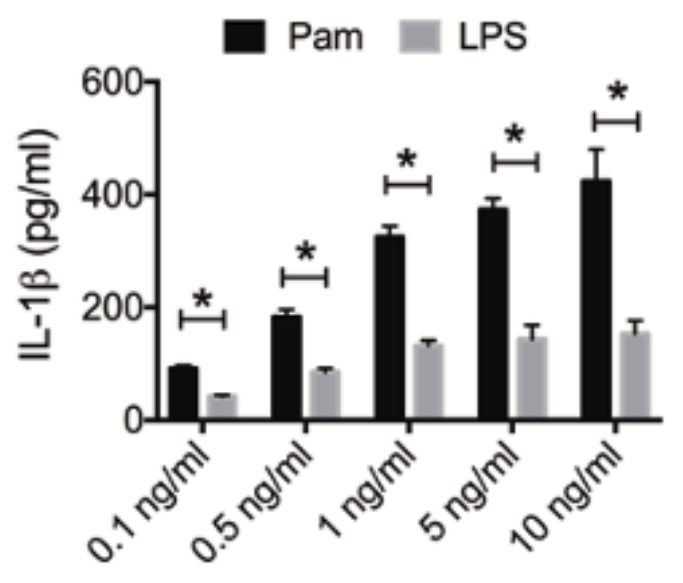

C

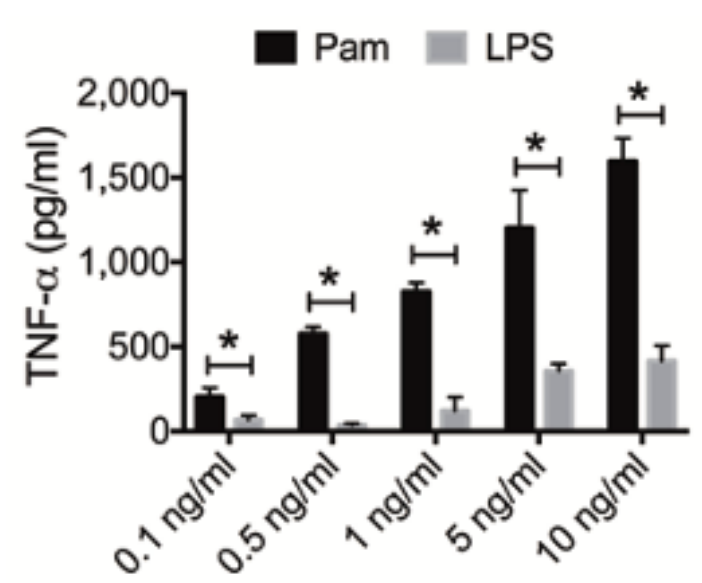

Fig. 2.1 Toll-like receptors 2 and 4 (TLR2 and TLR4) and CD44 receptor expression in THP-1 macrophages and impact of TLR receptor stimulation on proinflammatory cytokine production. Data represents the mean \pm standard deviation of 4 independent experiments. ${ }^{*} p<0.001$

A) A representative flow cytometry histogram of human THP-1 macrophages demonstrating enhanced TLR2 and CD44 receptor expression, compared to TLR4.

B) Impact of Pam3CSK4 (Pam; TLR2 ligand) and Lipopolysaccharide (LPS; TLR4 ligand) treatments on interleukin-1 beta (IL-1 $\beta$ ) production in THP-1 macrophages. 
C) Impact of Pam3CSK 4 and LPS treatment on tumor necrosis factor alpha (TNF- $\alpha$ ) production in THP-1 macrophages.

A

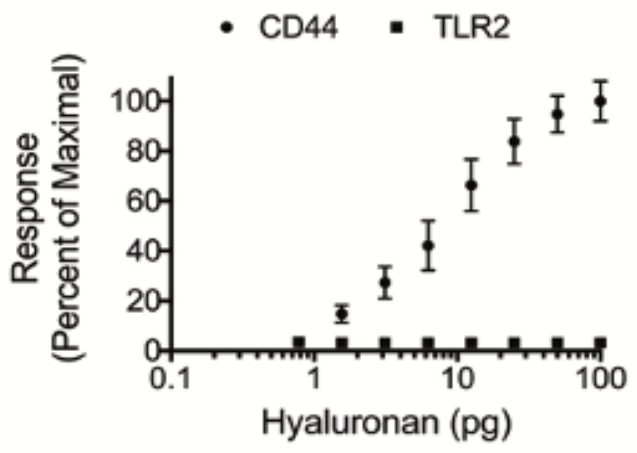

C

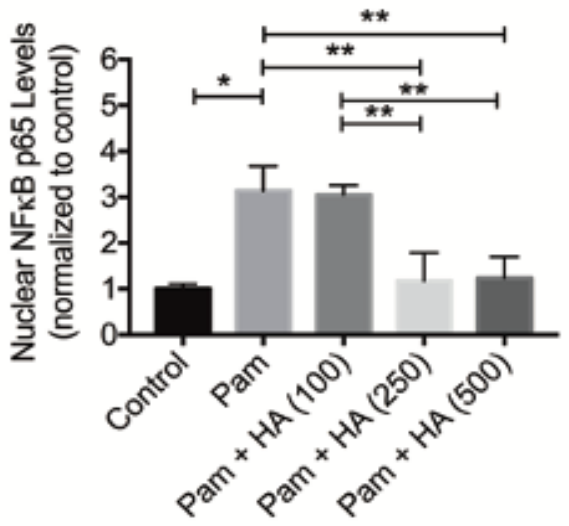

B

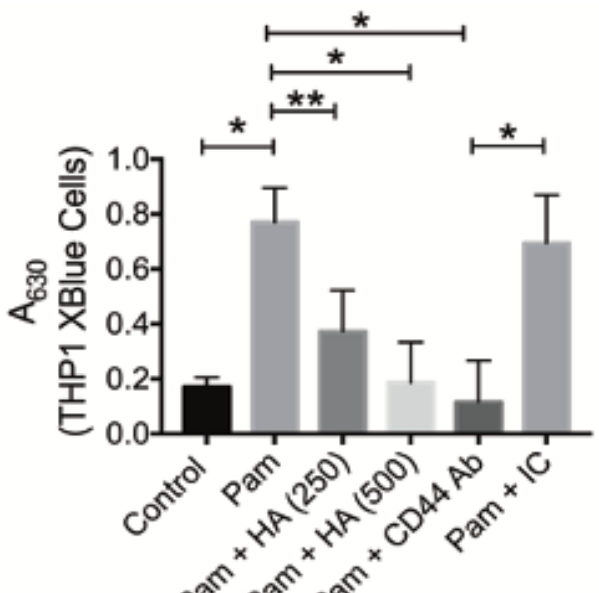

D

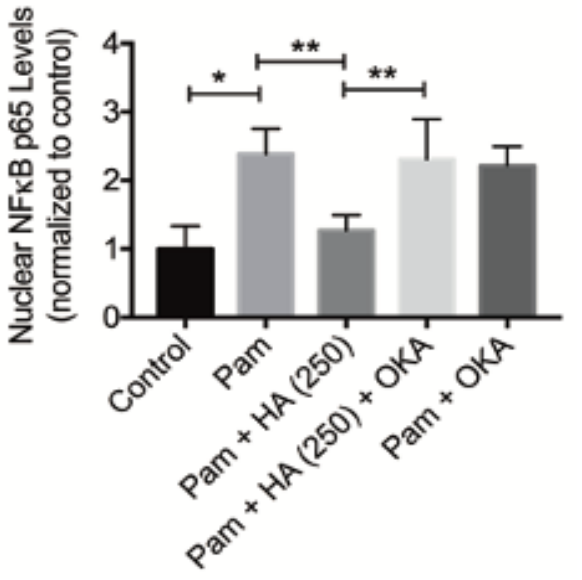

Fig. 2.2 Binding of hyaluronan (HA) to recombinant human CD44 and TLR2 receptors and impact of HA treatment on TLR2 receptor stimulated nuclear translocation of nuclear factor kappa B (NFkB). TLR2 receptor stimulation was performed using Pam3CSK4 (Pam; 5 ng/mL).

A) Concentration-dependent binding of HA to immobilized CD44 or TLR2 receptors using a microplate assay format. HA exhibited a concentration-dependent binding to CD44 receptor and no significant binding to TLR2 receptor.

B) Impact of HA treatment (250 and $500 \mu \mathrm{g} / \mathrm{mL})$, a CD44 antibody (CD44 Ab $2.5 \mu \mathrm{g} / \mathrm{mL}$ ) or an isotype control (IC; $2.5 \mu \mathrm{g} / \mathrm{mL}$ ) on TLR2 induced NFKB nuclear translocation in THP1 XBlue monocytes. HA and CD44 Ab treatments reduced TLR2 induced NFKB nuclear translocation. 
Data represents the mean \pm standard deviation of 4 independent experiments. $\quad * p<0.001$; $* * p<0.01$.

C) Impact of HA treatment $(100,250$ and $500 \mu \mathrm{g} / \mathrm{mL})$ on TLR2 induced NFKB p65 subunit nuclear translocation in THP-1 macrophages. HA $(250$ and $500 \mu \mathrm{g} / \mathrm{mL})$ treatments reduced NFkB nuclear translocation in THP-1 macrophages. Data represents the mean \pm standard deviation of 4 independent experiments. ${ }^{*} p<0.001 ; * *^{*}<0.01$.

D) Protein phosphatase 2A (PP2A) mediates HA's inhibition of NFkB p65 subunit nuclear translocation in THP-1 macrophages. Okadaic acid (OKA; $5 \mathrm{nM})$, a potent inhibitor of PP2A, abolished the inhibitory effect of HA $(250 \mu \mathrm{g} / \mathrm{mL})$ on NFKB p65 subunit translocation in THP-1 macrophages. Data represents the mean \pm standard deviation of 4 independent experiments. $* p<0.001 ; * * p<0.01$.

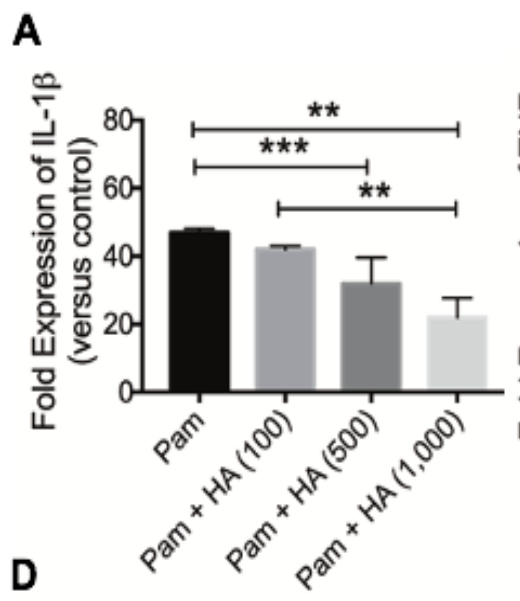

B

C
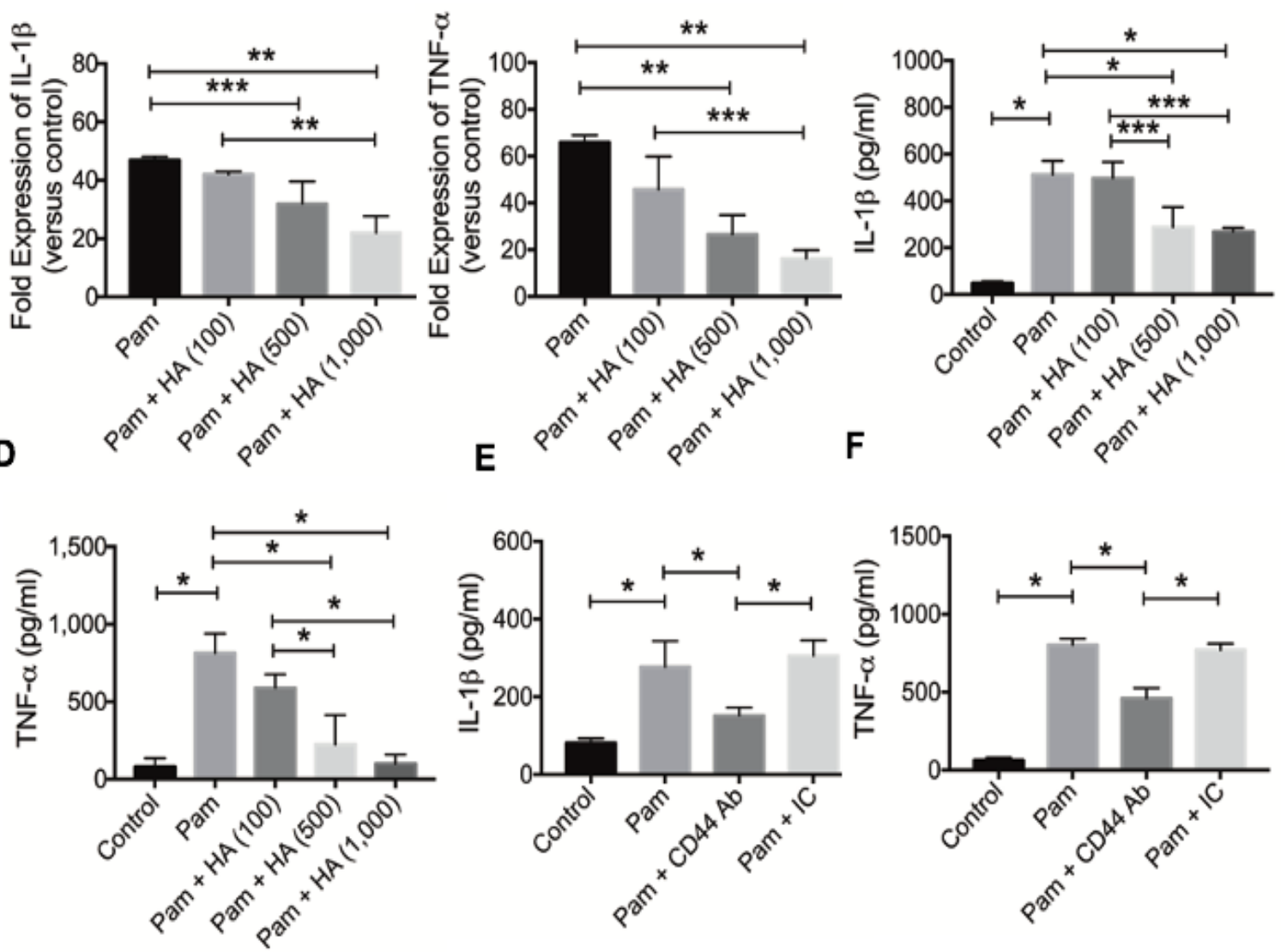

Fig. 2.3 Impact of hyaluronan (HA) (100, 500 and 1,000 $\mu \mathrm{g} / \mathrm{mL})$, CD44 antibody (CD44 Ab; 2.5 $\mu \mathrm{g} / \mathrm{mL}$ ) or isotype control (IC; $2.5 \mu \mathrm{g} / \mathrm{mL}$ ) treatments on TLR2 receptor induced interleukin-1 beta (IL-1 $\beta$ ) or tumor necrosis factor alpha (TNF $\alpha$ ) gene expression and production in THP- 
macrophages. Data represents the mean \pm standard deviation of 4 independent experiments. $*_{p}<0.001 ; * * p<0.01 ; * * * p<0.05$.
A) HA (500 and 1,000 $\mu \mathrm{g} / \mathrm{mL})$ treatments reduced TLR2 induced IL-1 $\beta$ gene expression.
B) HA (500 and 1,000 $\mu \mathrm{g} / \mathrm{mL})$ treatments reduced TLR2 induced TNF- $\alpha$ gene expression.
C) HA (500 and 1,000 $\mu \mathrm{g} / \mathrm{mL})$ treatments reduced TLR2 induced IL-1 $\beta$ production.
D) HA (500 and 1,000 $\mu \mathrm{g} / \mathrm{mL})$ treatments reduced TLR2 induced TNF- $\alpha$ production.
E) CD44 Ab treatment reduced TLR2 induced IL-1 $\beta$ production.
F) CD44 Ab treatment reduced TLR2 induced TNF- $\alpha$ production. 
A
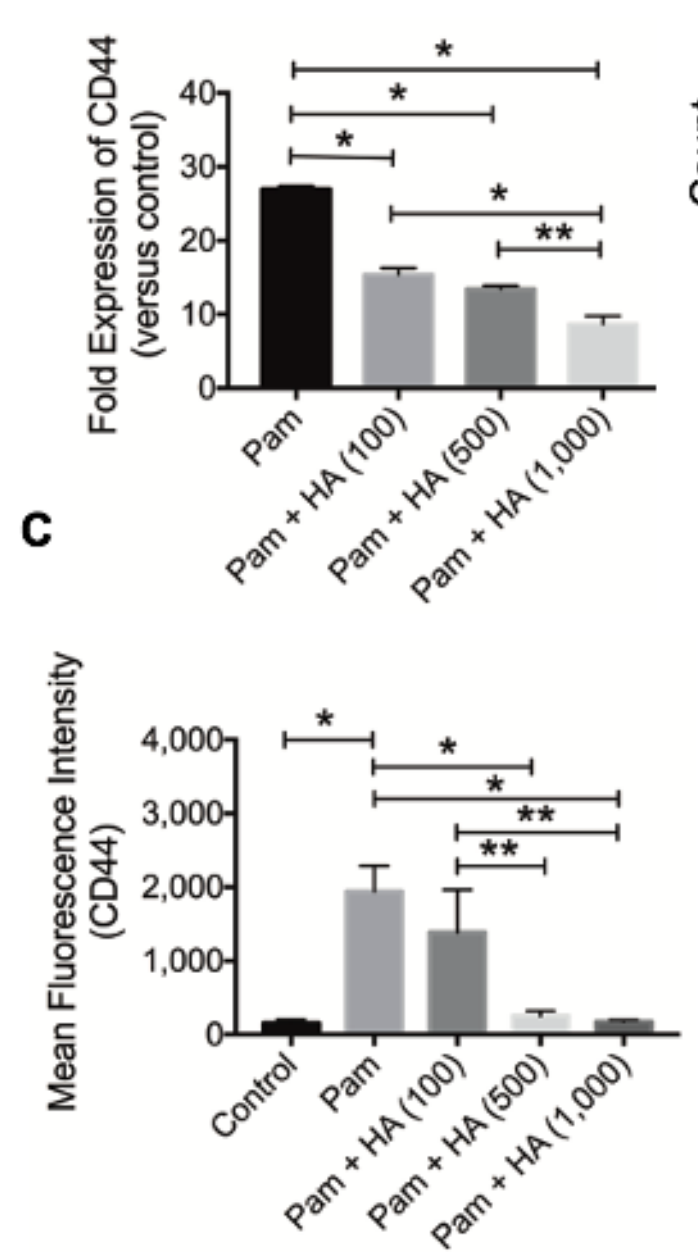

B $250-$

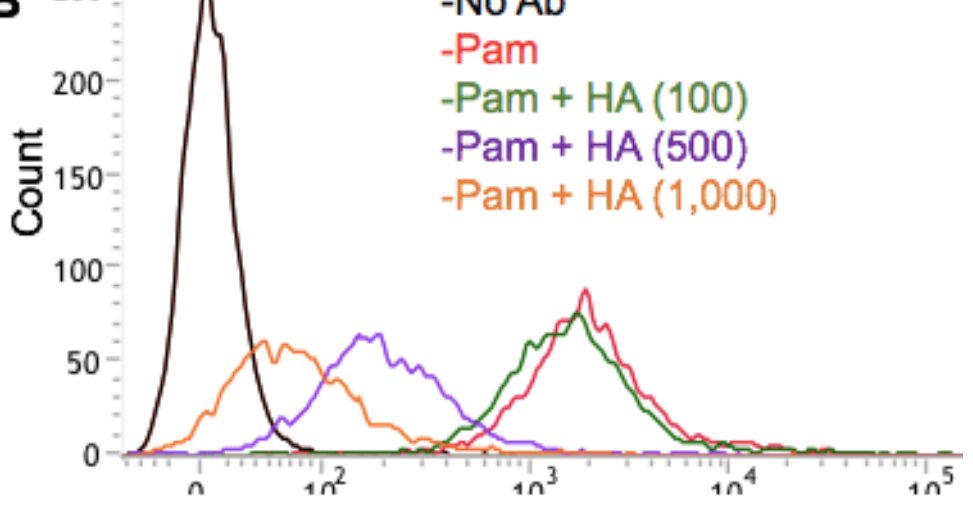

Fluorescence Intensity

Fig. 2.4 Impact of toll-like receptor 2 (TLR2) receptor stimulation on CD44 gene expression and CD44 levels in THP-1 macrophages and the role of hyaluronan (HA). TLR2 receptor stimulation was performed using Pam3CSK4 $(\mathrm{Pam} ; 5 \mathrm{ng} / \mathrm{mL})$ for 24 hours. Data represents the mean \pm standard deviation of 4 independent experiments. ${ }^{*} p<0.001 ; * * p<0.01$.

A) $\mathrm{HA}(100,500$ and $1,000 \mu \mathrm{g} / \mathrm{mL})$ treatments reduced TLR2 induced CD44 gene expression in THP-1 macrophages.

B) A representative flow cytometry histogram demonstrating increased CD44 protein levels in TLR2 stimulated THP-1 macrophages. HA $(500$ and $1,000 \mu \mathrm{g} / \mathrm{mL})$ treatments reduced CD44 levels in TLR2 stimulated THP-1 macrophages.

C) Semi-quantitative analysis of mean fluorescence intensities of CD44 receptor following TLR2 stimulation in the absence or presence of HA $(100,500$ or $1,000 \mu \mathrm{g} / \mathrm{mL})$. TLR2 stimulation 
increased CD44 protein in THP-1 macrophages and HA (500 and 1,000 $\mu \mathrm{g} / \mathrm{mL})$ treatments reduced CD44 protein.
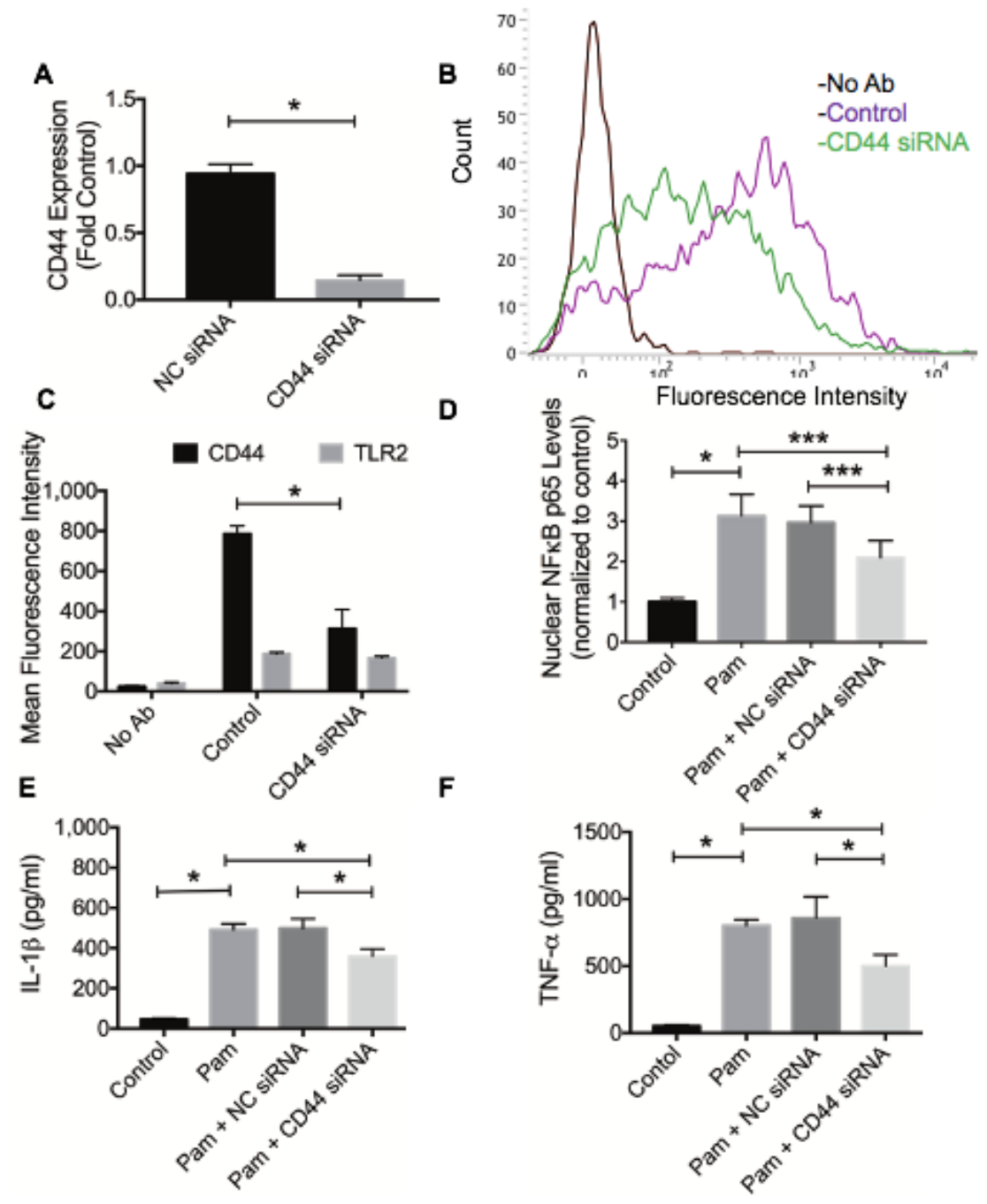

Fig. 2.5 Impact of CD44 receptor knockdown on toll-like receptor 2 (TLR2) receptor stimulated proinflammatory cytokine production in THP-1 macrophages and nuclear factor kappa B (NFאB) p65 subunit nuclear translocation. TLR2 receptor stimulation was performed using Pam3CSK4 (Pam; 5ng/mL). Data represents the mean \pm standard deviation of 4 independent experiments. $* p<0.001$. *** $p<0.05$.

A) CD44 siRNA treatment resulted in reduced CD44 gene expression compared to negative control (NC) siRNA treatment or control. 
B) A representative flow cytometry histogram demonstrating reduced CD44 protein levels in CD44 siRNA-treated THP-1 macrophages compared to control.

C) Semi-quantitative analysis of mean fluorescence intensities of CD44 or TLR2 receptors in THP-1 macrophages following CD44 knockdown. CD44 knockdown resulted in approximately $60 \%$ reduction in CD44 levels.

D) CD44 siRNA treatment reduced TLR2 stimulated NFאB p65 subunit nuclear translocation.

E) CD44 siRNA treatment reduced TLR2 stimulated interleukin-1 beta (IL-1 $\beta$ ) production in THP-1 macrophages.

F) CD44 siRNA treatment reduced TLR2 stimulated tumor necrosis factor alpha (TNF- $\alpha$ ) production in THP-1 macrophages.
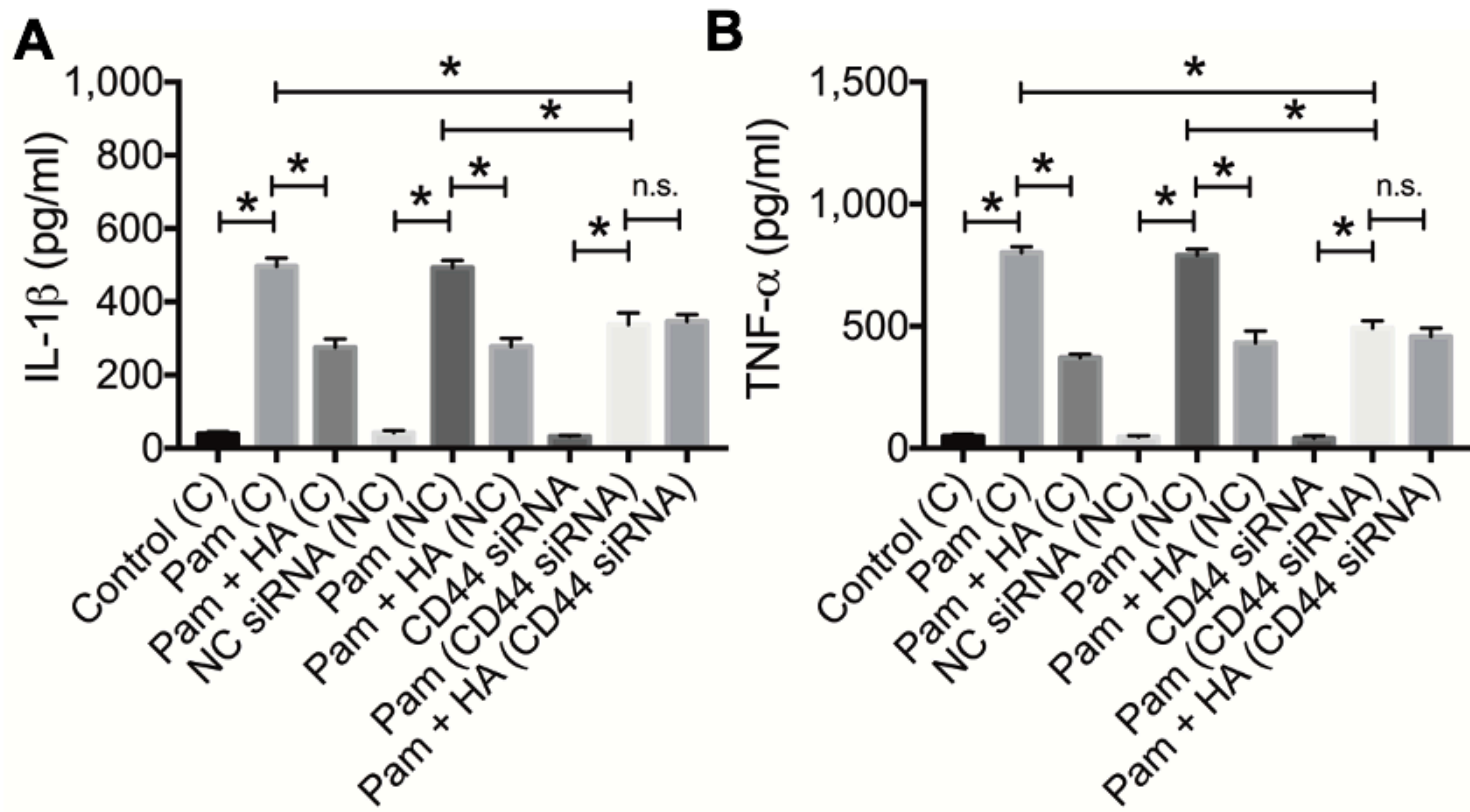

Fig. 2.6 Impact of hyaluronan (HA) treatment on toll-like receptor 2 (TLR2) induced interleukin1 beta (IL-1 $\beta$ ) and tumor necrosis factor alpha (TNF- $\alpha$ ) production in CD44 siRNA-treated, negative control (NC) siRNA-treated and untreated control THP-1 macrophages. TLR2 stimulation was performed using Pam3CSK4 (Pam; 5ng/mL) for 24 hours. HA treatment was performed at $500 \mu \mathrm{g} / \mathrm{mL}$. Data represents the mean \pm standard deviation of 4 independent experiments. ${ }^{*} p<0.001 ;$ n.s.: not significant.

A) HA treatment reduced TLR2 induced IL- $1 \beta$ production in untreated control and NC siRNAtreated THP-1 macrophages. HA treatment did not alter IL-1 $\beta$ levels in CD44 siRNA-treated macrophages. 
B) HA treatment reduced TLR2 induced TNF- $\alpha$ production in untreated control and NC siRNAtreated THP-1 macrophages. HA treatment did not alter TNF- $\alpha$ levels in CD44 siRNA-treated macrophages.

A

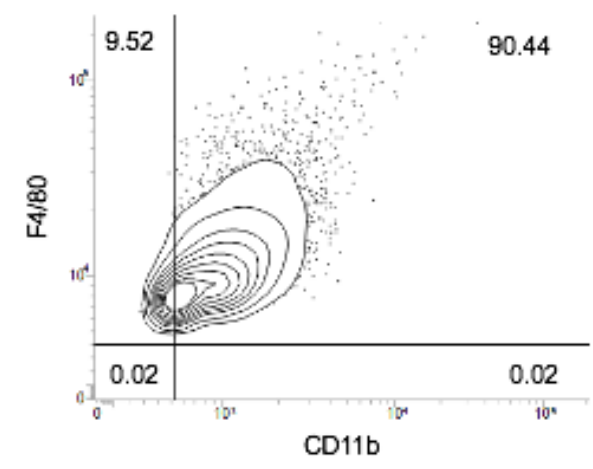

C

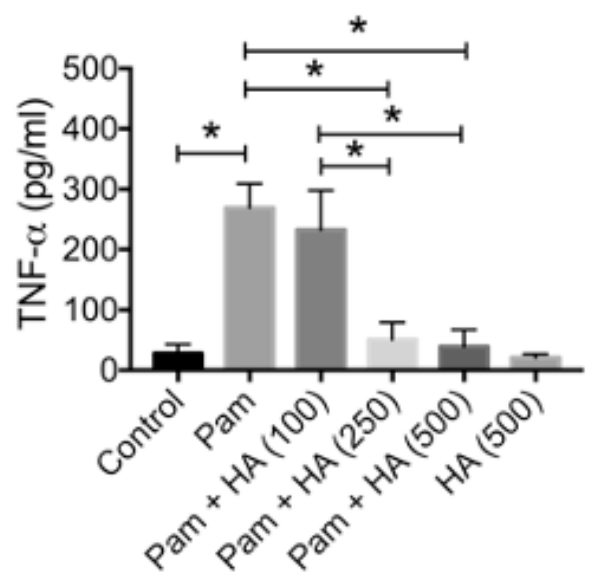

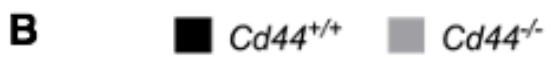

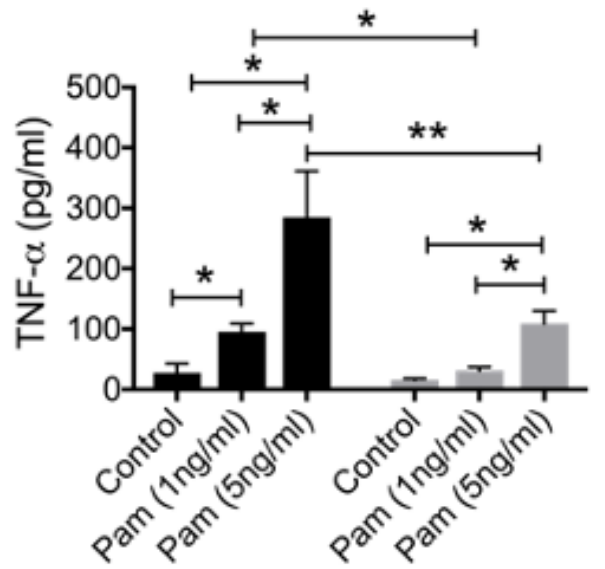

Fig. 2.7 Impact of toll-like receptor 2 (TLR2) receptor stimulation on tumor necrosis factor alpha (TNF- $\alpha$ ) production by $C D 44^{+/+}$and $C D 44^{-/}$murine bone marrow derived macrophages (BMDMs) and dose-dependent effect of HA on $C D 44^{+/+}$BMDMs. TLR2 stimulation was performed using Pam3CSK 4 (Pam; 5ng/mL) for 6 hours. Data represents the mean \pm standard deviation of 4 independent experiments. ${ }^{*} p<0.001 ;{ }^{* *} p<0.01$.

A) A representative flow cytometry histogram of murine BMDMs showing positive staining for $\mathrm{CD} 11 \mathrm{~b}$ and $\mathrm{F} 4 / 80$ surface markers.

B) TLR2 receptor activation dose-dependently increased TNF- $\alpha$ production by murine $C D 44^{+/+}$ BMDMs compared to $C D 44^{-/-} \mathrm{BMDMs}$. 
C) HA (250 and $500 \mu \mathrm{g} / \mathrm{mL})$ treatments reduced TLR2 induced TNF- $\alpha$ production by murine CD $44^{+/+}$BMDMs.
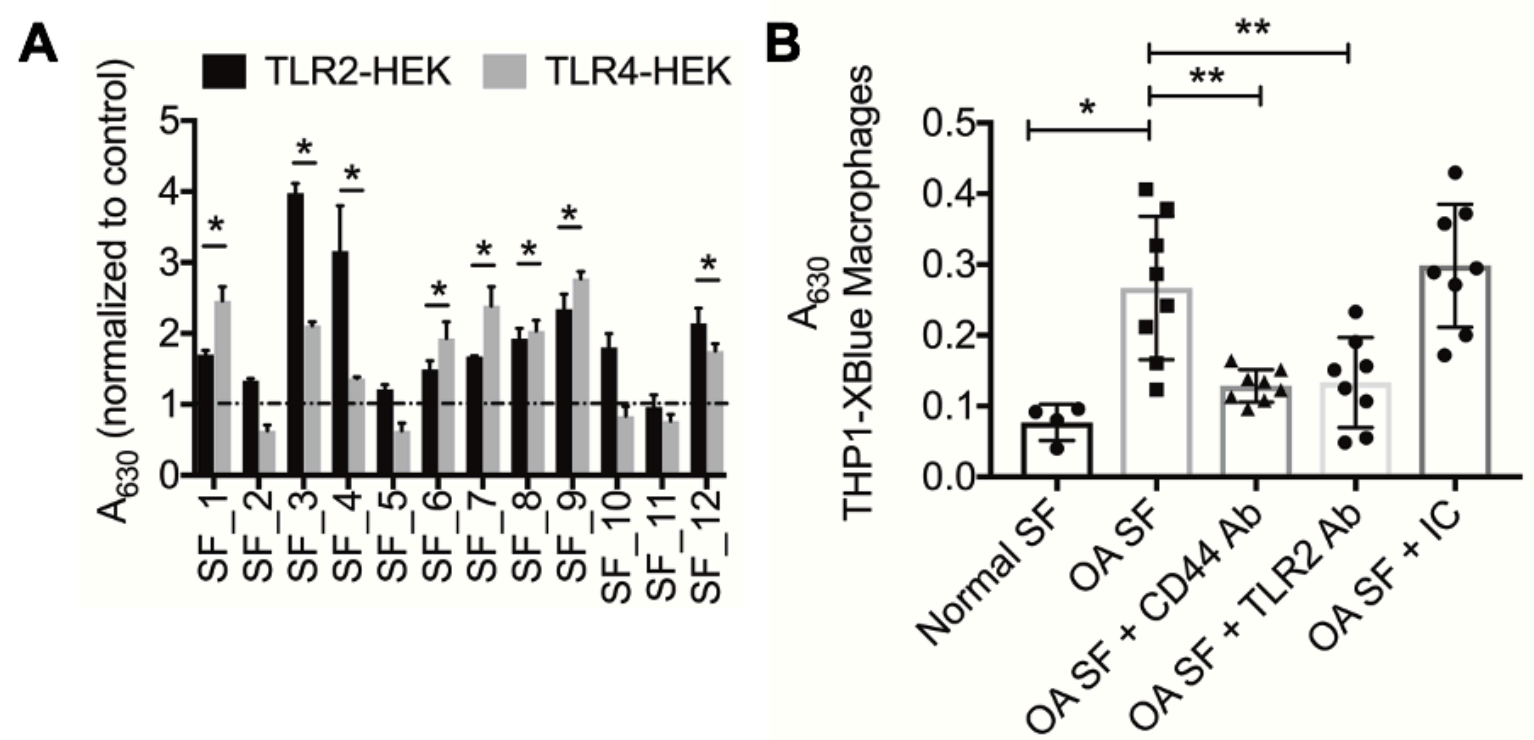

Fig. 2.8 Activation of toll-like receptors 2 and (TLR2 and TLR4) by synovial fluid (SF) aspirates from patients with advanced osteoarthritis (OA) and role of CD44 in modulating THP-1 macrophages activation by OA SF. OA SF $(3.75 \mu \mathrm{L}$ per well) were incubated with TLR2-HEK or TLR4-HEK cells in HEK detection media for 48 hours followed by measuring the $630 \mathrm{~nm}$ absorbance. THP-1XBlue macrophages were incubated with OA SF in the absence or presence of a TLR2-specific, CD44-specific, or isotype control (IC) antibody $(2.5 \mu \mathrm{g} / \mathrm{mL})$ for 48 hours followed by measuring $630 \mathrm{~nm}$ absorbance. Normal SF specimens were used as controls. $* p<0.001 ; * * p<0.01$.

A) Activation of TLR2 and TLR4 receptors by OA SF $(n=12)$. A total of 8 samples activated both TLR2 and TLR4 receptors, compared to untreated controls.

B) Activation of THP-1XBlue macrophages by OA SF $(n=8)$ and the impact of CD44-specific or TLR2-specific antibody treatments. CD44 and TLR2 antibody treatments reduced OA SF induced macrophage activation. 


\section{CHAPTER 3}

cAMP Attenuates TGF- $\beta$ 's Profibrotic Responses in Osteoarthritic Synoviocytes: Involvement of Hyaluronan and PRG4

Authors: Marwa M. Qadri, Gregory D. Jay, Rennolds S Ostrom, Ling X. Zhang, Khaled A. Elsaid

The following chapter was published in Am J Physio Cell Physiol. 2018 Sep 1;315(3):C432C443. doi: 10.1152/ajpcell.00041.2018. 
ABSTRACT

Osteoarthritis (OA) is characterized by synovitis and synovial fibrosis. Synoviocytes are fibroblast-like resident cells of the synovium that are activated by TGF- $\beta$ to proliferate, migrate and produce extracellular matrix. Synoviocytes secrete hyaluronan (HA) and proteoglycan-4 (PRG4). HA reduced synovial fibrosis in vivo and the $\operatorname{Prg}^{-/-}$mouse exhibits synovial hyperplasia. We investigated the antifibrotic effects of increased intracellular cAMP in TGF- $\beta$ stimulated human OA synoviocytes. TGF- $\beta 1$ stimulated collagen I (COL1A1), $\alpha$-SMA, TIMP-1, PLOD2 expression and procollagen I, $\alpha$-SMA, HA and PRG4 production, migration and proliferation of OA synoviocytes were measured. Treatment of OA synoviocytes with forskolin $(10 \mu \mathrm{M})$ increased intracellular cAMP levels and reduced TGF- $\beta 1$ stimulated COL1A1, $\alpha-$ SMA and TIMP-1 expression, with no change in PLOD2 expression. Forskolin also reduced TGF- $\beta 1$ stimulated procollagen I and $\alpha$-SMA content, as well as synoviocyte migration and proliferation. Forskolin $(10 \mu \mathrm{M})$ increased HA secretion, PRG4 expression and production. A cell permeable cAMP analog reduced COL1A1 and $\alpha$-SMA expression and enhanced HA and PRG4 secretion by OA synoviocytes. HA and PRG4 reduced $\alpha$-SMA expression and content and PRG4 reduced COL1A1 expression and procollagen I content in OA synoviocytes. $\operatorname{Prg} 4^{-/-}$synovium exhibited increased a-SMA, COL1A1 and TIMP-1 expression as compared to $\operatorname{Prg}^{+/+}$synovium. $\operatorname{Prg}^{-/-}$synoviocytes demonstrated strong $\alpha$-SMA and collagen type I staining while these were undetected in $\operatorname{Prg} 4^{+/+}$ synoviocytes, and was reduced with PRG4 treatment. We conclude that increasing intracellular cAMP levels in synoviocytes mitigates synovial fibrosis through enhanced production of HA and PRG4, possibly representing a novel approach for treatment of OA synovial fibrosis.

Keywords: cAMP, fibrosis, PRG4, HA, Osteoarthritis, synoviocytes. 


\subsection{INTRODUCTION}

Hallmarks of osteoarthritis (OA) include cartilage degeneration, subchondral bone remodeling, and synovitis $[112,118,156]$. Major abnormalities in the OA synovium include synovial hyperplasia, inflammatory cell infiltration, angiogenesis and fibrosis [157-160]. Synovial fibrosis is a common feature in advanced OA that contributes to joint pain and stiffness $[110,161]$. The TGF- $\beta 1$ family and its associated signaling pathways play an essential role in maintaining homeostasis in healthy joints [162]. However, TGF- $\beta 1$ switches to a pathologic role in OA joints that drives synovial fibrosis [163]. TGF- $\beta 1$ upregulates the expression of synovial collagen type 1, tissue inhibitor of metalloproteinase 1 (TIMP-1), and procollagen-lysine, 2-oxoglutarate 5dioxygenase 2 (PLOD2) $[163,164]$. The net result is an increase in collagen I formation, an increase in collagen crosslinks and a reduction in collagen turnover [163, 164].

The normal synovium contains two types of intimal cells: type A macrophages and type B fibroblasts, or synoviocytes [115]. In synoviocytes, TGF- $\beta 1$ induces fibrotic changes characterized by cell proliferation and collagen type I accumulation [165]. Additionally, TGF- $\beta 1$ promotes the differentiation of OA synoviocytes into a myofibroblast-like phenotype, characterized by the expression of alpha smooth muscle actin ( $\alpha$-SMA) [166]. Synoviocytes produce hyaluronan (HA), a glycosaminoglycan synthesized by membrane-bound hyaluronan synthase (HAS) with three isoforms identified to date (HAS1, HAS2 and HAS3) [167]. Synoviocytes also produce proteoglycan-4 (PRG4), a heavily glycosylated mucinous glycoprotein $[168,169]$. HA and PRG4 play important roles in joint lubrication [170]. HA and PRG4 may also

play a role in joint fibrosis as HA treatment reduced synovial fibrosis in vivo and findings in 
synovial tissues from $\operatorname{Prg} 4^{-/}$mice include increased synovial thickening and a proliferative capacity for $\operatorname{Prg}^{-/}$synoviocytes under basal and cytokine stimulated conditions [81, 92, 154].

Cyclic adenosine monophosphate (cAMP) is a pleiotropic intracellular second messenger generated by adenylyl cyclase (AC) enzymes in response to G-protein coupled receptor (GPCR) activation [62]. The antifibrotic effect of cAMP has been described in fibroblasts from multiple origins and include inhibition of fibroblast proliferation, reduction in fibroblast migration and reduced synthesis of extracellular matrix components [171-174]. The role of cAMP in regulating TGF- $\beta 1$ 's fibrotic response in synoviocytes is unknown. Our aim was to study the impact of forskolin, an AC activator, on the expression and production of $\alpha$-SMA, collagen type I, and expression of TIMP-1, PLOD2, HAS isoforms, PRG4 and production of HA and PRG4 in a model

of TGF- $\beta 1$ stimulated OA synoviocytes. Given the potential involvement of HA and PRG4 in synovial fibrosis, we also studied the antifibrotic effect of HA and PRG4 in human and murine synoviocytes. We hypothesized that increasing intracellular cAMP exerts an antifibrotic effect in OA synoviocytes and promotes HA and PRG4 production.

\subsection{MATERIALS AND METHODS}

\subsubsection{Ethical approvals:}

Animal breeding and tissue harvest was approved by the IACUC committee at Rhode Island Hospital.

\subsubsection{Patient characteristics and experimental approach}

OA synoviocytes (500,000 cells per vial; Cell Applications, USA) were isolated from synovial tissues from de-identified OA patients undergoing knee replacement $(\mathrm{n}=10$; median age $=63$; range: 54 to 69). Six patients were female. Five patients were Caucasian, and the other five were 
unspecified. Synoviocytes were received in their second passage. OA Synoviocytes were cultured in $75 \mathrm{~cm}^{2}$ culture flasks in DMEM media supplemented with $10 \% \mathrm{FBS}$ and were used between the third and sixth passages to avoid alterations in patterns of gene expression $[175,176]$. Experimental data are represented as the mean \pm S.D. of 3-6 biological replicates.

We initially studied cAMP generation in OA synoviocytes using a 0.01 to $10 \mu \mathrm{M}$ forskolin concentration range. The $10 \mu \mathrm{M}$ was selected based on previous studies [177, 178]. Following confirmation of cAMP accumulation by forskolin, we determined its effect on $\alpha$-SMA, collagen type I, TIMP-1 and PLOD2 expression in TGF- $ß 1$ stimulated OA synoviocytes. The antifibrotic effect of intracellular cAMP accumulation in OA synoviocytes was further confirmed using a cell permeable cAMP analog, 8-bromo cAMP. To further appreciate the antifibrotic effect of forskolin, we evaluated the impact of cAMP generation on HAS isoform expression and HA production, as well as PRG4 expression and production. Based on forskolin's observed effect on HA and PRG4, we studied the antifibrotic effect of HA and PRG4 in human OA synoviocytes, murine $\operatorname{Prg} 4^{-/}$synoviocytes as well as fibrosis markers in the $\operatorname{Prg} 4^{-/}$synovium. Finally, we studied the efficacy of forskolin in mitigating TGF- $\beta 1$ stimulated OA synoviocyte migration and proliferation.

\subsection{3 cAMP generation in OA synoviocytes by forskolin}

cAMP levels were measured in OA synoviocytes using the cADDis assay (Montana Molecular, USA). The assay utilizes a fluorescent cAMP sensor that measures changes in intracellular cAMP levels. An increase in intracellular cAMP levels results in a reduction in green fluorescence. OA synoviocytes $(10,000$ cells per well) were cultured overnight in sterile 96 -well clear-bottom black plates in the presence of a recombinant mammalianized baclovirus expressing the cAMP sensor 
according to manufacturer's recommendations. Subsequently, media supernatants were replaced with Dulbecco's phosphate-buffered saline (DPBS; Thermo Fisher Scientific, USA) (200 $\mu$ L per well). Forskolin (Sigma-Aldrich, USA) at a final concentration of $0.01,0.1,1$, and $10 \mu \mathrm{M}$, vehicle and a positive control were added and fluorescence intensity using 494/522 nm wavelengths was measured every 30 seconds over 30 minutes. Data is presented as the ratio of fluorescence intensity reduction at each time point to fluorescence intensity at baseline.

\subsubsection{Gene expression studies}

OA synoviocytes $(300,000$ cells per well) were treated with TGF- $\beta 1(1 \mathrm{ng} / \mathrm{mL}$; R\&D systems, USA) in serum-free DMEM \pm forskolin $(0.1,1$, or $10 \mu \mathrm{M}), 8$-bromo cAMP (Sigma Aldrich) (100 and $500 \mu \mathrm{M}$ ), human synoviocyte PRG4 (apparent MW $280 \mathrm{kDa}$ as a monomer; 100 $\mu \mathrm{g} / \mathrm{mL}$ ) [179] and/or high molecular weight HA (MW >950 kDa; R\&D Systems) (100 $\mu \mathrm{g} / \mathrm{mL})$ for 24 hours followed by RNA extraction, cDNA synthesis and qPCR as previously described [155]. The cycle threshold $(\mathrm{Ct})$ value of target genes were normalized to the $\mathrm{Ct}$ value of GAPDH in the same sample, and the relative expression was calculated using the $2^{-\Delta \Delta \mathrm{Ct}}$ method [134]. Target genes included a-SMA (ACTA2), collagen type I (COL1A1), TIMP-1, PLOD2, HAS1, HAS2, HAS3, and PRG4 (primers and probes were obtained from Thermo Fisher Scientific). Data are presented as fold expression of target genes in the different experimental groups compared to untreated controls.

\subsection{5 $\alpha$-SMA and procollagen type I quantitation}

OA synoviocytes were seeded in cell culture dishes $\left(20.8 \mathrm{~cm}^{2}\right)$ at $1.0 \times 10^{6}$ cells per dish until confluence. OA synoviocytes in serum-free DMEM media ( $5 \mathrm{ml}$ per dish) were treated with TGF$\beta 1(1 \mathrm{ng} / \mathrm{mL}) \pm$ forskolin $(10 \mu \mathrm{M})$, PRG4 $(100 \mu \mathrm{g} / \mathrm{mL})$ or HA $(100 \mu \mathrm{g} / \mathrm{mL})$ for 24 hours. Cell protein extraction was performed using M-PER reagent supplemented with protease and phosphatase 
cocktail inhibitor (Thermo Fisher Scientific) and quantified using micro BCA assay (Thermo Fisher Scientific).

Gel electrophoresis was performed using 10\% PAGE gels (Bio-Rad) and 10 $\mu$ g protein per well. Following transfer, membranes were blocked with 5\% non-fat dry milk for 2 hours at room temperature. Membranes were probed with anti- $\alpha$-SMA (1:1,000 dilution) and anti-GAPDH (1:5,000 dilution) (Abcam) overnight in tris-buffered saline tween 20 (TBS-T). Following washing with TBS-T, membranes were incubated with horseradish peroxidase (HRP)-conjugated anti-rabbit (1:5,000 dilution) antibody for 1 hour at room temperature (Abcam). Protein bands were developed using Lumigen ECL Ultra reagent (Lumigen, USA) and visualized using Bio-Rad ChemiDoc XRS+ system (Bio-Rad). Bands of interest were selected and quantified using Image $\mathrm{J}$ software. The ratio of $\alpha$-SMA band intensities to corresponding GAPDH band intensities of the different experimental groups were calculated and normalized to controls.

Procollagen I content in OA synoviocytes was determined using an ELISA (Abcam and R\&D Systems). A total of $5 \mu \mathrm{g}$ protein in $100 \mu \mathrm{L}$ buffer was used in each experimental group and procollagen I concentrations $(\mathrm{pg} / \mathrm{mL})$ were divided by 50 and expressed as procollagen I protein per $\mu \mathrm{g}$ protein.

\subsubsection{Immunocytostaining of $\alpha$-SMA in OA synoviocytes}

OA synoviocytes (200,000 cells per well) were cultured on collagen type I-coated $12 \mathrm{~mm}$ glass coverslips for 48 hours in DMEM supplemented with 10\% FBS. Subsequently, cells were treated with TGF- $\beta 1(1 \mathrm{ng} / \mathrm{ml}) \pm$ forskolin $(10 \mu \mathrm{M})$ for 48 hours in serum-free DMEM. Synoviocytes were fixed in $10 \%$ neutral buffered formalin for 10 min followed by washing twice with PBS. Cells 
were permeabilized for 5 min using $0.01 \%$ Triton X100 in PBS and blocked using 2\%BSA for 1 hour at room temperature. Probing was performed using FITC-conjugated anti $\alpha$-SMA antibody (1:100; Abcam) and Alexa Fluor 594 conjugated anti-alpha tubulin antibody (1:500; Abcam) overnight at $4{ }^{\circ} \mathrm{C}$. Following washing with PBS, cells were mounted with DAPI mounting medium (Abcam) for 1 hour and viewed under a confocal microscope.

\subsubsection{HA, PRG4 and HAS1 quantitation}

HA concentrations: OA synoviocytes (300,000 cells per well) in serum-free DMEM were treated with TGF- $\beta 1(1 \mathrm{ng} / \mathrm{mL}) \pm$ forskolin $(10 \mu \mathrm{M})$ for 24 hours. Media supernatants were collected and assayed for HA using a quantitative assay kit (R\&D systems). In a separate set of experiments, OA synoviocytes $(300,000$ cells per well) in serum-free DMEM were treated with TGF- $\beta 1$ $(1 \mathrm{ng} / \mathrm{mL}) \pm 8$-bromo cAMP $(100$ and $500 \mu \mathrm{M})$ for 24 hours. HA concentrations were determined as described above.

PRG4 concentrations: OA synoviocytes $(20,000$ cells per well) were seeded in sterile 96 well plates for 48 hours followed by treatment with TGF- $\beta 1(1 \mathrm{ng} / \mathrm{mL}) \pm$ forskolin $(10 \mu \mathrm{M})$ for 48 hours. PRG4 concentrations, normalized to cell density, were determined in media supernatants as previously described [155]. In a separate set of experiments, OA synoviocytes $(300,000$ cells per well) in serum-free DMEM were treated with TGF- $\beta 1(1 \mathrm{ng} / \mathrm{mL}) \pm 8$-bromo cAMP (100 and $500 \mu \mathrm{M})$ for 24 hours. PRG4 concentrations were determined as described above.

HAS1 content: OA synoviocytes were seeded and treated as described for $\alpha$-SMA and procollagen type I. HAS1 content in protein isolates were determined using an ELISA (MyBioSource, USA). A total of $1 \mu \mathrm{g}$ total protein in $100 \mu \mathrm{L}$ volume was added to the wells of the ELISA plate. HAS1 
concentrations $(\mathrm{ng} / \mathrm{mL})$ were divided by 10 and expressed as HAS1 content (ng) per $\mu \mathrm{g}$ total protein.

\subsubsection{HAS1 knockdown and its impact on forskolin-induced HA secretion in OA synoviocytes}

OA synoviocytes $(300,000$ cells per well) in Opti-MEM reduced serum medium (Thermo Fisher Scientific) were treated with a HASl small interfering RNA (siRNA) (Thermo Fisher Scientific) ( 25 pmoles per well) or a non-targeted negative control (NC) siRNA ( 25 pmoles per well) (Thermo Fisher Scientific) for 48 hours. Transfection was performed using Lipofectamine RNAiMAX (Thermo Fisher Scientific) per manufacturer's recommendations. To confirm HASl knockdown, HAS1 expression was determined as described above. In a separate set of experiments, HAS1 knockdown in OA synoviocytes was performed followed by media change to serum-free DMEM and stimulation with TGF- $\beta 1(1 \mathrm{ng} / \mathrm{mL}) \pm$ forskolin $(10 \mu \mathrm{M})$ for 24 hours. Subsequently, HA concentrations were determined in media supernatants as described above.

\subsubsection{Gene expression studies in $\operatorname{Prg}^{+/+}$and $\operatorname{Prg} 4^{-/}$synovial tissues and immunocytostaining of murine synoviocytes}

The phenotype of the $\operatorname{Prg}^{-/-}$mouse has been previously reported [180], and is characterized by cartilage degeneration and a hyperplastic synovium contributing to joint failure [180]. The Prg4${ }^{\prime}$ and $\operatorname{Prg} 4^{+/+}$mouse colonies are maintained by Dr. Jay at Rhode Island Hospital. $\operatorname{Prg} 4^{-/}$mouse is also commercially available (stock \#025737; The Jackson Laboratory, Maine, USA). Synovial tissues were isolated from male $\operatorname{Prg} 4^{-/-}$and $\operatorname{Prg} 4^{+/+}$mice (8-10 weeks old). The skin and surrounding tissues of the knee joints were removed. The joint capsule was cut open along both sides of the patella under a stereo microscope and the synovium from the lateral and medial sides was carefully isolated. A total of $15 \operatorname{Prg}^{+/+}$and $15 \operatorname{Prg}^{-/ /}$male mice were used in this study. Synovial tissues were harvested from every mouse and tissues from 3 mice were randomly pooled 
into one sample, generating 5 pooled samples in each genotype. RNA isolation, cDNA synthesis and qPCR were performed as previously described. Genes of interest included ACTA2, COL1A1, TIMP-1 and PLOD2 with GAPDH as an internal reference gene (Thermo Fisher Scientific).

$\mathrm{Prg}^{+/+}$and $\operatorname{Prg}^{-/ /}$synoviocytes were isolated as previously described [86]. Synovial tissues from male $\operatorname{Prg} 4^{+/+}$and $\operatorname{Prg}^{-/}$mice (15 animals per genotype) were used to isolate the synoviocytes. Synoviocytes were plated onto sterile chamber slides (Thermo Fisher Scientific) at a density of 1.0 x $10^{6}$ cells per well and allowed to adhere for 24 hrs. Synoviocytes were incubated with human synoviocyte PRG4 (100 $\mu \mathrm{g} / \mathrm{mL})$ in serum-free DMEM for 24 hours followed by washing with PBS and cell fixation with $4 \%$ formalin. Probing was performed using anti- $\alpha$-SMA antibody (1:100 dilution) or anti-collagen type I antibody (1:200 dilution) (Abcam) at $4^{\circ} \mathrm{C}$ overnight. Following washing with PBS, cells were incubated with Cy3 goat anti-rabbit IgG antibody (1:200 dilution; Thermo Fisher Scientific) for $1 \mathrm{hr}$ at room temperature in the dark. Following washing with PBS, Alexa Fluor 488-conjugated phalloidin, a filamentous actin (F-actin) probe (1:125 dilution; Thermo Fisher Scientific), was added for $20 \mathrm{~min}$ in the dark. Cells were subsequently mounted with DAPI mounting medium (Vector Labs) and viewed under a fluorescent microscope (Nikon E 800).

\subsubsection{Basal and TGF- $\beta 1$ induced OA synoviocytes proliferation and migration}

In sterile 96 well plates, OA synoviocytes $(10,000$ cells per well) were cultured in serum-free DMEM media and incubated with forskolin $(3,10$ and $30 \mu \mathrm{M}) \pm \mathrm{TGF}-\beta 1(1 \mathrm{ng} / \mathrm{mL})$ for 48 hours at $37^{\circ} \mathrm{C}$. Cell proliferation was determined using the MTT reagent (Sigma). OA synoviocytes $(100,000$ cells per well) were seeded in 24 -well culture plates in DMEM+10\% FBS for 72 hours. A $1,000 \mu \mathrm{L}$ pipette tip was used to perform a scratch in the confluent synoviocyte monolayer. 
TGF- $\beta 1(1 \mathrm{ng} / \mathrm{mL})$ stimulation was performed in serum-free DMEM \pm forskolin $(10 \mu \mathrm{M})$ for 48 hours. Subsequently, media was aspirated and cells were stained (Cell Biolabs, USA) followed by imaging using all-in-one fluorescence microscope (Keyence, USA). A region of interest (ROI) was defined and the scratch width was measured at multiple locations in the ROI. The mean scratch width was calculated and used to estimate the mean scratch area. Data is presented as the ratio of the scratch areas of the different experimental groups to the scratch area at baseline.

\subsubsection{Statistical Analyses}

Variables were initially tested for normality. Normally distributed variables were compared using Student's $t$-test for two groups or analysis of variance with Tukey's post-hoc test for more than two groups. Variables that did not satisfy the normality assumption were tested using ANOVA on the ranks. Statistical analysis of gene expression data was performed using $\Delta \mathrm{Ct}$ values $\left(\mathrm{C}_{\mathrm{t}}\right.$ target gene- $C_{t}$ GAPDH) for each gene of interest. Significance level was set at 0.05.

\subsection{RESULTS}

3.3.1 Forskolin treatment increased intracellular cAMP, reduced $A C T A 2, C O L 1 A 1, T I M P-1$ expression and reduced $\alpha-S M A$ and procollagen type $I$ in TGF- $\beta 1$ stimulated OA synoviocytes

A representative dose-response of forskolin is shown in figure 3.1A. Treatment with forskolin $(0.01 \mu \mathrm{M})$ did not increase intracellular cAMP while the $0.1,1$ and $10 \mu \mathrm{M}$ forskolin treatments resulted in detectable increases in cAMP. Forskolin $(10 \mu \mathrm{M})$ increased intracellular cAMP compared to vehicle (fig. $3.1 B ; p<0.001)$ ( $\mathrm{n}=3$ patients). TGF- $\beta 1$ induced ACTA2, COL1A1, TIMP-1 and PLOD2 expression (fig. 3.1C through $F ; p<0.001$ versus control for the 4 genes) $(\mathrm{n}=4$ patients). Forskolin treatment reduced ACTA2 (fig. 3.1C; $p<0.001$ ), COL1A1 (fig. 3.1D; $p<0.01$ ), and TIMP-1 (fig. 3.1E; $p<0.001$ ) expression compared to TGF- $\beta 1$ alone. Forskolin treatment did 
not alter TGF- $\beta 1$ stimulated $P L O D 2$ expression (fig. $3.1 F ; p=0.833$ ). Forskolin did not alter basal COL1A1 $(p=0.623)$, TIMP-1 $(p=0.802)$ or PLOD2 $(p=0.752)$ expression. In contrast, forskolin reduced basal $A C T A 2$ expression $(p=0.018)$.

A Western Blot and semi-quantitative analysis of $\alpha$-SMA using GAPDH as a loading control are shown in fig. $3.1 \mathrm{G}$ and fig. $3.1 \mathrm{H}$, respectively. TGF- $\beta 1$ increased $\alpha$-SMA protein in OA synoviocytes compared to control (fig. 3.1H; $p<0.001)$ ( $\mathrm{n}=6$ patients). Forskolin reduced TGF- $\beta 1$ stimulated $\alpha$-SMA production $(p=0.013)$. Forskolin alone did not alter basal $\alpha$-SMA content $(p=0.660)$. Representative confocal images of TGF- $\beta 1$-treated OA synoviocytes \pm forskolin is shown in figure 3.1I. Control OA synoviocytes exhibited a positive $\alpha$-SMA staining, and the appearance of a myofibroblast-like phenotype in a number of cells. TGF- $\beta 1$ treatment resulted in stronger $\alpha$-SMA staining and the appearance of myofibrils and this was markedly reduced with forskolin co-treatment. TGF- $\beta 1$ increased procollagen type I protein compared to control (fig. $3.1 \mathrm{~J} ; \mathrm{p}<0.01)(\mathrm{n}=3$ patients). Forskolin reduced TGF- $\beta 1$ linked procollagen type I production $(p=0.015)$, and did not alter basal procollagen type I $(p=0.991)$.

\subsubsection{Forskolin treatment enhanced HA secretion and modulated HAS isoform gene expression and $H A S 1$ knockdown attenuated forskolin's effect on HA production in TGF- $\beta 1$ stimulated OA synoviocytes}

HA concentrations were higher in TGF- $\beta 1$ treated OA synoviocytes and forskolin-treated OA synoviocytes compared to untreated controls (fig. 3.2A; $p<0.001$ for both comparisons) ( $\mathrm{n}=4$ patients). HA concentrations in the TGF- $\beta 1+$ forskolin group were higher than HA concentrations in the TGF- $\beta 1$ or forskolin alone groups ( $p<0.001$ for both comparisons). TGF- $\beta 1$ induced HAS1 (fig. 3.2B; $p<0.001$ ) and HAS2 (fig. 3.2C; $p<0.001)$ with no effect on HAS3 (fig. 3.2D; $p=0.719$ ) expression in OA synoviocytes ( $\mathrm{n}=4$ patients). Forskolin treatment upregulated basal HAS1, HAS2 and HAS3 expression ( $p<0.001$ for all comparisons). Combined treatment of TGF- $\beta 1$ and 
forskolin increased HAS1 expression over TGF- $\beta 1$ alone (fig. 3.2B; $p=0.026$ ). In contrast, HAS2 expression in the TGF- $\beta 1+$ forskolin group was lower than HAS 2 expression in the TGF- $\beta 1$ group (fig. 3.2C; $p=0.024)$.

The total cellular HAS1 content was higher in TGF- $\beta 1(p=0.021)$ and TGF- $\beta 1+$ forskolin $(p<0.01)$ treatments compared to control (fig. 3.2E) ( $\mathrm{n}=4$ patients). HAS1 content was not different between TGF- $\beta 1+$ forskolin and TGF- $\beta 1$ alone groups $(p=0.971)$. Similarly, there was no difference in HAS1 between forskolin and control groups $(p=0.303)$. HAS1 expression was reduced by approximately $68 \%$ in OA synoviocytes transfected with $H A S 1$ siRNA (fig. $3.2 F$; $p<0.01)$ ( $\mathrm{n}=4$ OA patients). HA concentrations in TGF- $\beta 1$ stimulated HAS1 knockdown OA synoviocytes were not different from unstimulated HAS1 knockdown OA synoviocytes (fig. 3.2G; $p=0.962)(\mathrm{n}=4$ patients). Similarly, HA concentrations in TGF- $\beta 1+$ forskolin treated HAS1 knockdown OA synoviocytes were not different from HA concentrations in TGF- $\beta 1$ treated HAS1 knockdown OA synoviocytes $(p=0.514)$. Finally, HA concentrations in TGF- $\beta 1+$ forskolin treated OA synoviocytes were higher than HA concentrations in TGF- $\beta 1+$ forskolin treated HAS1 knockdown OA synoviocytes $(p<0.001)$.

\subsubsection{Forskolin treatment enhanced PRG4 expression and secretion in TGF- $\beta 1$ stimulated OA synoviocytes}

TGF- $\beta 1$ induced PRG4 expression (fig. 3.3A; $p<0.001$ ) and increased PRG4 production by OA synoviocytes $(f i g .3 .3 B ; p<0.01)(\mathrm{n}=4$ patients). Forskolin did not alter basal $P R G 4$ expression (fig. 3.3A; $p=0.063$ ) or PRG4 production (fig. 3.3B; $p=0.996$ ) in OA synoviocytes. PRG4 expression in the TGF- $\beta 1+$ forskolin group was higher than TGF- $\beta 1$ alone (fig. 3.3A; $p=0.037$ ). Correspondingly, PRG4 concentrations were higher in the TGF- $\beta 1+$ forskolin group compared to the TGF- $\beta 1$ group (fig. 3.3B; $p=0.031$ ). 


\subsubsection{Impact of PRG4 and HA treatments on $A C T A 2$ and $C O L 1 A 1$ expression and $\alpha-S M A$ and procollagen type $I$ in TGF- $\beta 1$ stimulated OA synoviocytes}

ACTA2 expression was lower in the TGF- $\beta 1+$ PRG4 group compared to TGF- $\beta 1$ alone (fig. 3.3C; $p<0.001)(\mathrm{n}=4$ patients). Similarly, $A C T A 2$ expression in the TGF- $\beta 1+$ HA and TGF- $\beta 1+$ PRG4 + HA groups was lower than ACTA2 expression in TGF- $\beta 1$ alone ( $p<0.001$ for both comparisons). COL1A1 expression was lower in the TGF- $\beta 1+$ PRG4 group compared to TGF- $\beta 1+$ HA $(p<0.01)$ or TGF- $\beta 1$ alone $(p<0.001)$ (fig. 3.3D) ( $\mathrm{n}=4$ OA patients). In contrast, HA treatment did not alter TGF- $\beta 1$ induced COL1A1 expression $(p=0.897)$. COL1A1 expression in the TGF- $\beta 1+$ PRG4 + HA group was lower than TGF- $\beta 1(p<0.001)$ and TGF- $\beta 1+$ HA $(p<0.01)$ groups.

A Western Blot and semi-quantitative analysis of $\alpha$-SMA using GAPDH as a loading control are shown in 3.3E and 3.3F, respectively. $\alpha-$ SMA content was lower in TGF- $\beta 1+$ PRG4 (fig. 3.3F; $p<0.01)$ and in TGF- $\beta 1+$ HA $(p<0.01)$ compared to TGF- $\beta 1$ alone ( $\mathrm{n}=4$ patients). Procollagen type I content was lower in TGF- $\beta 1+$ PRG4 compared to TGF- $\beta 1$ alone $(f i g .3 .3 G ; p<0.01)(\mathrm{n}=4$ patients). There was no difference in procollagen type I content between TGF- $\beta 1+$ HA and TGF$\beta 1$ groups $(p=0.059)$.

3.3.5 A cell permeable cAMP analog treatment reduced $A C T A 2$ and $C O L 1 A 1$ expression and enhanced $H A$ and PRG4 secretion in TGF- $\beta 1$ stimulated OA synoviocytes

TGF- $\beta 1$ induced ACTA2 and COL1A1 expression in OA synoviocytes (fig. 3.4A and 3.4B; $p<0.001$ against control for both genes). 8-bromo cAMP $(100 \mu \mathrm{M})$ treatment did not significantly alter TGF- $\beta 1$ induced $A C T A 2$ and COL1A1 expression ( $p>0.05$ for both comparisons). In contrast, 8-bromo cAMP $(500 \mu \mathrm{M})$ treatment reduced ACTA2 $(p<0.001)$ and COL1A1 $(p<0.001)$ expression in TGF- $\beta 1$ stimulated OA synoviocytes ( $\mathrm{n}=3$ OA patients). HA and PRG4 media concentrations in TGF- $\beta 1+8$-bromo cAMP $(500 \mu \mathrm{M})$ group were significantly higher than 
corresponding concentrations in TGF- $\beta 1$ only group (fig. $3.4 \mathrm{C}$ and $3.4 \mathrm{D} ; p<0.001$ for both comparisons) ( $\mathrm{n}=3$ OA patients).

\subsubsection{ACTA2, COL1A1 and TIMP-1 expression was higher in $\mathrm{Prg}^{4-/}$ synovial tissues and human synoviocyte PRG4 treatment reduced $\alpha$-SMA and collagen type I staining in Prg $4^{-/-}$ synoviocytes}

Expression of ACTA2 ( $p=0.021)$, COL1A1 $(p<0.001)$ and TIMP-1 $(p<0.01)$ was higher in Prg4-

/ synovia compared to $\mathrm{Prg}^{+/+}$synovia (fig. 3.5A). In contrast, PLOD2 expression was lower in $\operatorname{Prg} 4^{-/}$tissues compared to $\operatorname{Prg} 4^{+/+}$tissues $(p<0.01)$.

Merged images of $\alpha$-SMA and collagen type I stained $\operatorname{Prg} 4^{+/+}$and $\operatorname{Prg} 4^{-/-}$synoviocytes is shown in figure 3.5B. We observed strong $\alpha$-SMA and collagen type I staining in $\operatorname{Prg}^{-/}$synoviocytes. $\alpha$-SMA staining co-localized with F-actin staining. In contrast, there was no detected $\alpha$-SMA or collagen type I staining in $\operatorname{Prg}^{+/+}$synoviocytes. PRG4 treatment reduced $\alpha$-SMA and collagen type I staining in $\operatorname{Prg}^{-/}$synoviocytes.

\subsubsection{Forskolin reduced TGF- $\beta 1$ induced OA synoviocyte proliferation and migration}

Forskolin $(3 \mu \mathrm{M}$ and $10 \mu \mathrm{M})$ treatments did not alter basal OA synoviocyte proliferation (fig. 3.6A; $p=0.891$ and $p=0.117) \quad(\mathrm{n}=4$ patients). In contrast, the $30 \mu \mathrm{M}$ treatment increased basal OA synoviocyte proliferation compared to untreated control $(p<0.01)$. TGF- $\beta 1$ stimulated OA synoviocyte proliferation (fig. $3.6 \mathrm{~B} ; p<0.001$ ) ( $\mathrm{n}=4$ patients). OA synoviocyte proliferation in the TGF- $\beta 1+$ forskolin $(30 \mu \mathrm{M})$ group was lower than TGF- $\beta 1$ alone $(p<0.001)$, TGF- $\beta 1+$ forskolin $(3 \mu \mathrm{M})(p<0.01)$ or TGF- $\beta 1+$ forskolin $(10 \mu \mathrm{M})(p<0.01)$. There was no difference in cell proliferation between TGF- $\beta 1+$ forskolin $(10 \mu \mathrm{M})$ and TGF- $\beta 1$ alone $(p=0.063)$. Representative wound scratch images are show in figure 3.6C. TGF- $\beta 1$ enhanced OA synoviocyte migration (fig. $3.6 D ; p<0.01)(\mathrm{n}=4$ patients). OA synoviocyte migration in the TGF- $\beta 1+$ forskolin $(10 \mu \mathrm{M})$ group 
was lower than in the TGF- $\beta 1$ only group $(p<0.01)$. There was no difference in cell migration between forskolin treated and untreated OA synoviocytes $(p=0.887)$.

\subsection{DISCUSSION}

In this paper, we show that TGF- $\beta 1$ resulted in excess collagen type I production, induction of TIMP-1 and PLOD2 expression and $\alpha$-SMA upregulation, together with stimulating OA synoviocyte migration and proliferation. Forskolin, by virtue of its ability to generate cAMP, reduced collagen production and blunted TIMP-1 expression while inhibiting synoviocyte proliferation and migration. PLOD2 induction in osteoarthritic synoviocytes is consistent with its established role in mediating synovial collagen crosslinking [181, 182]. Forskolin did not alter PLOD2 expression, which may be related to the TGF- $\beta 1$ 's signaling pathways. Remst et al have shown that in OA synoviocytes, an ALK 1/2/3/6 inhibitor completely blocked TGF- $\beta 1$ induced collagen type I expression whereas TGF- $\beta 1$ induced PLOD2 expression was only slightly reduced [183].

OA synoviocytes proliferate in response to various mitogenic stimuli $[155,184,185]$. In our experiments, TGF- $\beta 1$ induced cell proliferation with a magnitude comparable to what has been previously reported [184]. Forskolin, at the treatment level that inhibited migration, exhibited a marginal antiproliferative effect and a higher concentration was needed to observe significant antagonism of TGF- $\beta 1$ 's mitogenic effect. This might be due to a low proliferative capacity of OA synoviocytes. In the absence of TGF- $\beta 1$, forskolin acted as a mitogen to produce a low, yet significant, stimulation of proliferation. Forskolin directly binds to AC and generates cAMP from ATP [186]. The increase in cAMP results in activation of protein kinase A (PKA) dependent and independent pathways $[186,187]$. In the presence of growth factors, forskolin activates cAMP- 
dependent PKA which interferes with Raf-1 activation and signaling to blunt cell proliferation [67]. PKA also activates CREB, which can compete for cofactors with SMAD-mediated transcription stimulated by TGF- $\beta 1[177]$. In the absence of growth factor, PKA-dependent and independent pathways stimulate cAMP-mediated cell proliferation [188].

We measured $\alpha$-SMA in OA synoviocytes from human patients and forskolin addition consistently attenuated TGF- $\beta 1$-stimulated $\alpha$-SMA expression. $\alpha$-SMA is a specific marker of myofibroblasts $[189,190]$. Myofibroblasts are effector cells in fibrosis that possess enhanced ability to produce collagen, proliferate and migrate $[189,190]$. We have shown that forskolin treatment appeared to markedly reduce TGF- $\beta 1$ 's induced myofibroblast-like phenotype in OA synoviocytes. The causal role that myofibroblasts may play in synovial fibrosis is understudied and unclear. Steenvoorden et al reported that $\alpha$-SMA staining was only found in blood vessels in synovia from healthy individuals [191]. Interestingly, TGF- $\beta 1$ stimulation of normal synoviocytes increased collagen type I expression with no effect on $\alpha$-SMA expression or production [191]. Mattey et al have shown that TGF- $\beta 1$ or IL-4 treatments trigger differentiation of OA synoviocytes into myofibroblast-like cells, characterized by $\alpha$-SMA expression in vitro [166]. Evidence relating myofibroblasts to changes occurring in joint fibrosis was reported by Sasabe et al [192]. Using a rat knee contracture model, myofibroblasts expressing $\alpha$-SMA were detected as early as 1 week from joint immobilization and this was associated with increased collagen type I expression and joint capsule fibrosis [192]. We have also detected $\alpha$-SMA protein in $\operatorname{Prg} 4^{-/ /}$synoviocytes with no $\alpha$-SMA signal in normal murine synoviocytes. The positive $\alpha$-SMA signal in $\operatorname{Prg}^{4^{-/}}$knockout synoviocytes is associated with phenotypical changes in the synovium including synovial lining thickening and enhanced synoviocyte proliferation [81]. 
TGF- $\beta 1$ induced $H A S 1$ and $H A S 2$ expression with no effect on $H A S 3$, and enhanced HA secretion by OA synoviocytes. Synoviocytes contain higher levels of HAS1 message compared to HAS2 with HAS3 being the least abundant [193]. Earlier reports are in agreement with our finding that TGF- $\beta 1$ stimulation of arthritic synoviocytes resulted in a higher HAS1/HAS2 transcript ratio above control level and that in turn resulted in higher extracellular HA levels [184, 193]. Our data suggests that the majority of TGF- $\beta 1$ linked HA secretion is mediated by HAS1, as HAS1 knockdown diminished extracellular HA concentrations. Co-treatment with forskolin increased the HAS1/HAS2 transcript ratio above the corresponding TGF-ß1 ratio with greater HA secretion over 24 hours. The increase in HAS1 mRNA in forskolin-treated synoviocytes did not translate to increased HAS1 cellular pool. This might be related to the rate at which the message is being translated. Furthermore, the majority of HAS1 cellular fraction is inactive and is found in the cytoplasm either diffused or partially co-localized with the Golgi apparatus, whereas the plasma membrane-bound fraction is small and is catalytically active [178]. Therefore, the possibility that the newly synthesized HAS1 enzyme, in response to forskolin treatment, could have been trafficked to the membrane resulting in increasing the rate of HA synthesis could not be ruled out. Assessing the impact of forskolin on membrane-bound HAS1 level and activity was technically challenging and was not feasible to perform. Our data should also be considered in the context that other factors e.g. post-translational modifications, availability of precursors and regulation of HAS activity will likely contribute to the amount of HA secreted by the OA synoviocytes[193] .

Increasing intracellular cAMP resulted in increasing PRG4 expression and production by OA synoviocytes in the setting of TGF- $\beta 1$ stimulation. This contextual effect is due to CREB stimulation which was previously shown to enhance PRG4 production by superficial zone articular chondrocytes [194]. PRG4 is a mucin-like glycoprotein synthesized by synoviocytes and 
superficial zone articular chondrocytes with a heavily glycosylated central domain and an $\mathrm{N}$ - and C-termini [195]. The mouse Prg4 gene is highly homologous to the human $\operatorname{Prg} 4$ gene [196]. Human and mouse Prg4 genes each consist of 12 exons and the N- and C-termini are highly conserved across species [196]. PRG4 exists in the synovial fluid in monomeric or multimeric forms and functions as a boundary lubricant $[195,197]$. PRG4 binds to CD44, the HA receptor, and exerts an anti-inflammatory effect in OA synoviocytes [86, 155]. PRG4 also acts in an autocrine manner to regulate OA synoviocyte proliferation [155]. We have found that PRG4 and HA had equivalent efficacy in reducing $\alpha$-SMA content in osteoarthritis synoviocytes. However, PRG4, at a physiologically-relevant concentration [150], was more efficacious than HA in reducing collagen I expression and production, indicative of a potential role in antagonizing profibrotic alterations in synovial tissues. This function is likely related to its interaction with the CD44 receptor, given that HA reduced synovial fibrosis in a CD44-mediated manner [92]. PRG4 reduced collagen I and $\alpha$-SMA staining in $\operatorname{Prg}^{-/}$synoviocytes. The link between PRG4 expression and synovial fibrosis is further illustrated by the upregulation of collagen type I, TIMP1 and $\alpha$-SMA in the $\operatorname{Prg}^{-/-}$synovium. As laying excess collagen type I is a prominent feature in synovial fibrosis, the strong immunocytostaining for collagen type I in $\operatorname{Prg} 4^{-/}$synoviocytes, coupled with other synovial changes, support a fibrotic $\operatorname{Prg}^{-/-}$synovium. We did not include human normal synoviocytes in our study design. Furthermore, we did not examine the efficacy of forskolin or PRG4 in an in-vivo model of synovial fibrosis.

In summary, our data demonstrate that forskolin; a diterpene produced by the roots of the Indian plant Coleus forskohili [186], increases intracellular cAMP levels and produces an antifibrotic effect in OA synoviocytes. Increasing intracellular cAMP levels directly via treatment with a cell permeable cAMP analog recapitulated the antifibrotic effect of forskolin. Forskolin reduces 
collagen type I expression and procollagen type I production and inhibits TGF- $\beta 1$ linked fibroblast migration and proliferation. Forskolin also increased HA and PRG4 secretion by OA synoviocytes; an effect that may contribute to its overall antifibrotic efficacy. Approaches that increase cAMP levels in synoviocytes can promote an antifibrotic phenotype and may be a novel approach for slowing the progression of synovial fibrosis in OA.

\subsection{GRANTS}

This work is supported by R01AR067748 to KE and GJ.

\subsection{DISCLOSURES}

Authors MQ, LZ and RO have nothing to disclose. GJ authored patents related to use of recombinant PRG4 and holds equity in Lubris LLC, MA, USA. KE co-authored patent applications related to use of recombinant PRG4.

\subsection{AUTHOR CONTRIBUTIONS}

Authors MQ, LZ and KE carried out the experiments and participated in data analysis. GJ participated in study design and critical interpretation of results. KE and RO conceived the study and participated in data analysis and interpretation. All authors participated in drafting and critical evaluation of the manuscript. All authors have read and approved the final version of the manuscript.

\subsection{ACKNOWLEDGEMENTS}

The authors would like to thank Austin Kazarian for his help with the cAMP assays. 


\subsection{FIGURES}

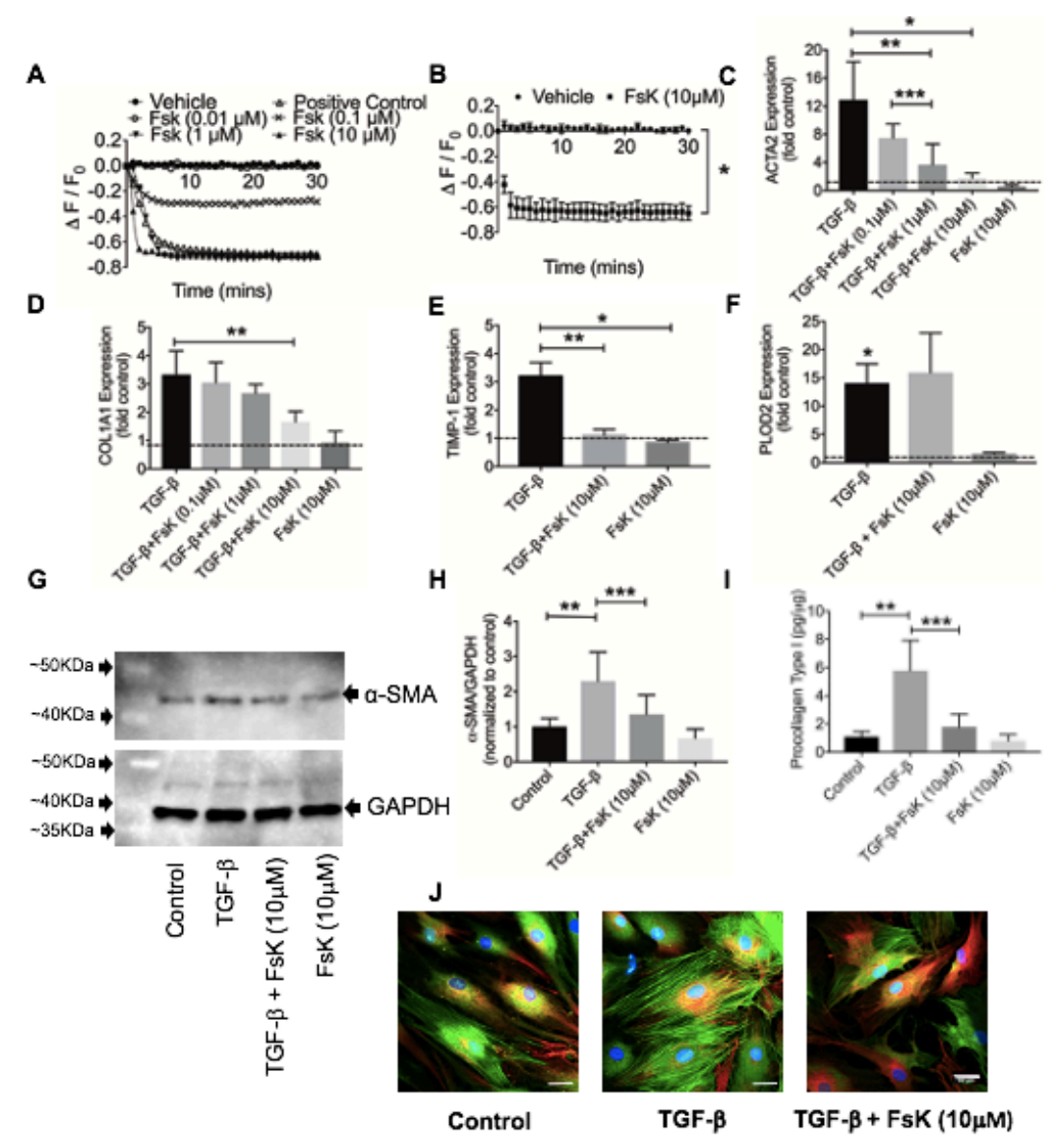

Fig. 3.1 Impact of forskolin (FsK) treatment on intracellular cyclic adenosine monophosphate (cAMP) levels, basal and transforming growth factor beta 1 (TGF- $\beta 1$ )-induced alpha smooth muscle actin (ACTA2), collagen I (COL1A1), tissue inhibitor of metalloproteinase 1 (TIMP-1) and procollagen-lysine, 2-oxoglutarate 5-dioxygenase 2 (PLOD2) gene expression and alpha smooth muscle action ( $\alpha$-SMA) and procollagen type I production in osteoarthritic (OA) synoviocytes. TGF- $\beta 1(1 \mathrm{ng} / \mathrm{ml})$ stimulation of OA-FLS was performed for 24 hours in all experiments except $\alpha$ SMA immunocytostaining (stimulation was performed using $1 \mathrm{ng} / \mathrm{ml}$ TGF- $\beta 1$ for 48 hours). Data is presented as the mean \pm S.D. of experiments utilizing OA synoviocytes from different patients. $*_{p}<0.001 ; * * p<0.01 ; * * * p<0.05$.

A. Representative dynamic change in intracellular cAMP levels in OA synoviocytes following treatment with FsK $(0.01,0.1,1$ and $10 \mu \mathrm{M})$. FsK treatment $(0.1,1$ and $10 \mu \mathrm{M})$ resulted in detectable cAMP levels in OA synoviocytes. The cAMP signal was detected using a cAMPspecific sensor. B. cAMP levels were elevated in FsK $(10 \mu \mathrm{M})$-treated OA synoviocytes $(\mathrm{n}=3$ patients). C. FsK treatment $(1$ and $10 \mu \mathrm{M})$ reduced TGF- $\beta 1$ induced ACTA2 expression $(\mathrm{n}=4$ 
patients). D. FsK treatment $(10 \mu \mathrm{M})$ reduced TGF- $\beta 1$ induced $C O L 1 A 1$ expression ( $\mathrm{n}=4$ patients). E. FsK treatment $(10 \mu \mathrm{M})$ reduced TGF- $\beta 1$ induced TIMP-1 expression ( $\mathrm{n}=4$ patients). F. Fsk treatment $(10 \mu \mathrm{M})$ did not alter TGF- $\beta 1$ induced PLOD2 expression ( $\mathrm{n}=4$ patients). G. Western Blot of $\alpha$-SMA in control, TGF- $\beta 1$, TGF- $\beta 1+$ FsK and FsK-treated OA synoviocytes. GAPDH was used as loading control. H. Semi-quantitative densitometry analysis of $\alpha$-SMA normalized to GAPDH and expressed as ratio to control in cell extracts of control, TGF- $\beta 1$, TGF- $\beta 1+$ FsK and FsK-treated OA synoviocytes. FsK $(10 \mu \mathrm{M})$ treatment reduced TGF- $\beta 1$ linked increase in $\alpha$-SMA in OA synoviocytes $(\mathrm{n}=6$ patients). I. FsK $(10 \mu \mathrm{M})$ treatment reduced $\alpha$-SMA staining and myofibroblast-like phenotype in TGF- $\beta 1$ stimulated OA synoviocytes. J. Procollagen type I content in cell extracts of control, TGF- $\beta 1$, TGF- $\beta 1+$ FsK and FsK-treated OA synoviocytes. Data was normalized to total protein content. FsK $(10 \mu \mathrm{M})$ treatment reduced TGF- $\beta 1$ linked increase in procollagen type I content in OA synoviocytes ( $\mathrm{n}=3$ patients).

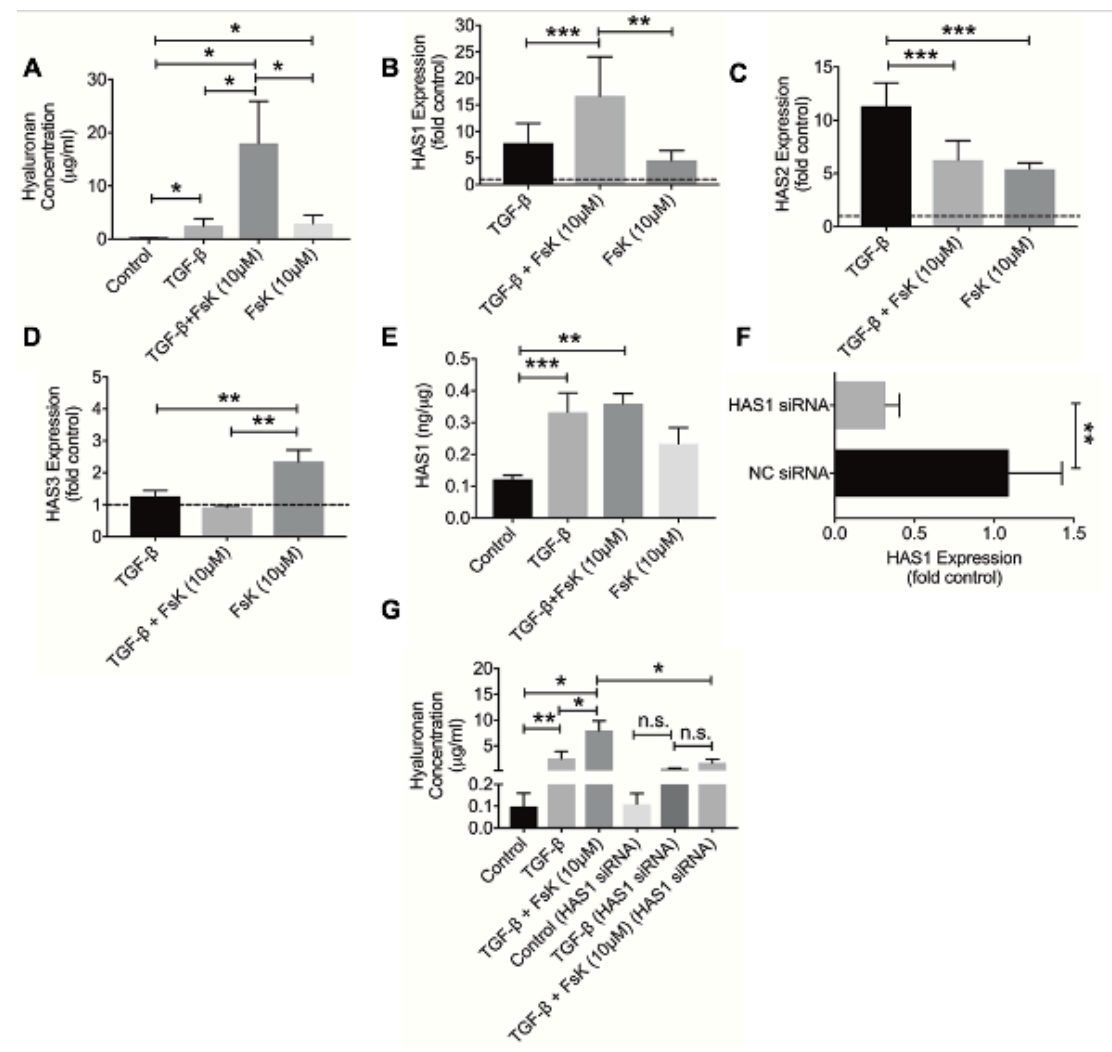

Fig. 3.2 Impact of forskolin $(\mathrm{FsK} ; 10 \mu \mathrm{M})$ treatment on basal and transforming growth factor beta 1 (TGF- $\beta 1$ )-induced hyaluronan (HA) production, expression of hyaluronan synthase isoforms 1 , 2, and 3 (HAS1, HAS2 and HAS3) and the role of HAS1 in mediating TGF- $\beta 1$ and FsK-linked HA production in osteoarthritic $(\mathrm{OA})$ synoviocytes. Data is presented as the mean \pm S.D. of experiments utilizing OA synoviocytes from 4 patients. ${ }^{*} p<0.001 ;{ }^{*} p<0.01 ;{ }^{*} *_{p}<0.05$; n.s.: non significant.

A. Hyaluronan concentrations in control, TGF- $\beta 1$, TGF- $\beta 1+$ FsK, and FsK-treated OA synoviocytes. B. FsK treatment enhanced TGF- $\beta 1$ induced $H A S 1$ expression. C. FsK treatment 
reduced TGF- $\beta 1$ induced $H A S 2$ expression. D. FsK treatment increased basal $H A S 3$ expression in OA synoviocytes. E. HAS1 protein content in cell extracts of control, TGF- $\beta 1$, TGF- $\beta 1+$ FsK and FsK-treated OA synoviocytes. Data was normalized to total protein content. There was no difference in HAS1 protein between TGF- $\beta 1$ and TGF- $\beta 1+$ FsK treatments. F. HAS1 expression was reduced in HAS1 siRNA-treated OA synoviocytes compared to untreated control and negative control siRNA (NC siRNA)-treated OA synoviocytes. G. FsK and/or TGF- $\beta 1$ treatments did not significantly change hyaluronan production following HAS1 knockdown in OA synoviocytes.

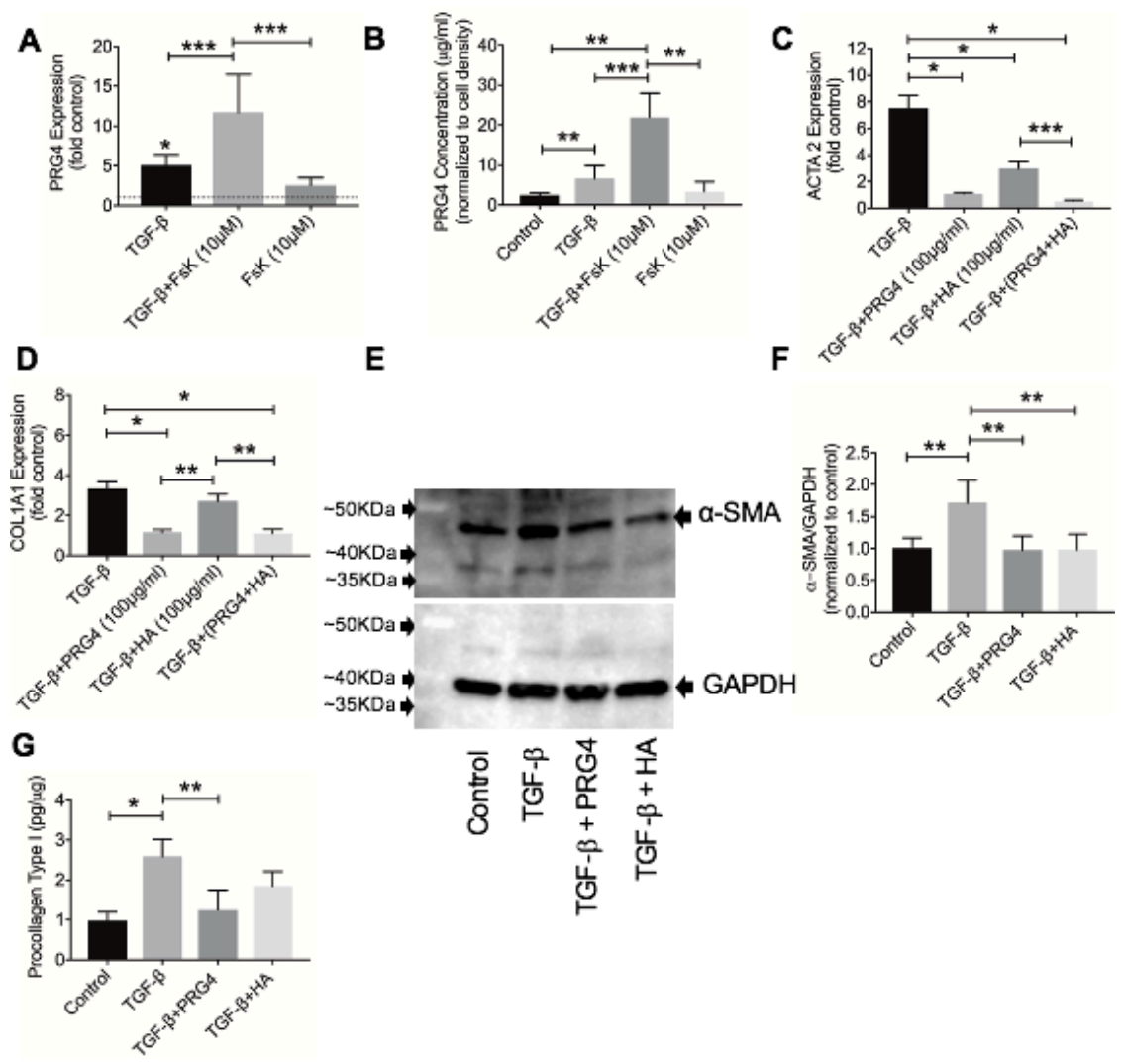

Fig. 3.3 Impact of forskolin $(\mathrm{FsK} ; 10 \mu \mathrm{M})$ treatment on basal and transforming growth factor beta 1 (TGF- $\beta 1$ ) induced proteoglycan-4 (PRG4) gene expression and production by osteoarthritic (OA) synoviocytes and efficacy of human synoviocyte PRG4 $(100 \mu \mathrm{g} / \mathrm{ml})$ and hyaluronan (HA) $(100 \mu \mathrm{g} / \mathrm{ml})$ in modulating TGF- $\beta 1$ induced expression and production of alpha smooth muscle actin and collagen type I in OA synoviocytes. Data is presented as the mean \pm S.D. of experiments utilizing OA synoviocytes from 4 patients. ${ }^{*} p<0.001 ; * * p<0.01 ; * * * p<0.05$.

A. TGF- $\beta 1$ increased PRG4 expression and FsK treatment enhanced TGF- $\beta 1$ 's effect. B. FsK treatment enhanced TGF- $\beta 1$ linked PRG4 production by OA synoviocytes. C. PRG4 and/or HA treatments reduced TGF- $\beta 1$ induced alpha smooth muscle actin gene (ACTA2) expression in OA synoviocytes. D. PRG4 treatment reduced TGF- $\beta 1$ induced collagen type I (COL1A1) gene expression in OA synoviocytes. E. Western Blot of $\alpha$-SMA in control, TGF- $\beta 1$, TGF- $\beta 1+$ PRG4 $(100 \mu \mathrm{g} / \mathrm{ml})$ and TGF- $\beta 1+\mathrm{HA}(100 \mu \mathrm{g} / \mathrm{ml})$ treated OA synoviocytes. GAPDH was used as loading control. F. Semi-quantitative densitometry analysis of $\alpha$-SMA normalized to GAPDH and 
expressed as ratio to control in cell extracts of control, TGF- $\beta 1$, TGF- $\beta 1+$ PRG4 and TGF- $\beta 1+$ HA treated OA synoviocytes. PRG4 and HA treatments reduced TGF- $\beta 1$ linked increase in $\alpha-$ SMA in OA synoviocytes. G. Procollagen type I content in cell extracts of control, TGF- $\beta 1$, TGF$\beta 1+$ PRG4 and TGF- $\beta 1+$ HA-treated OA synoviocytes. Data was normalized to total protein content. PRG4 treatment reduced TGF- $\beta 1$ linked increase in procollagen type I content in OA synoviocytes.

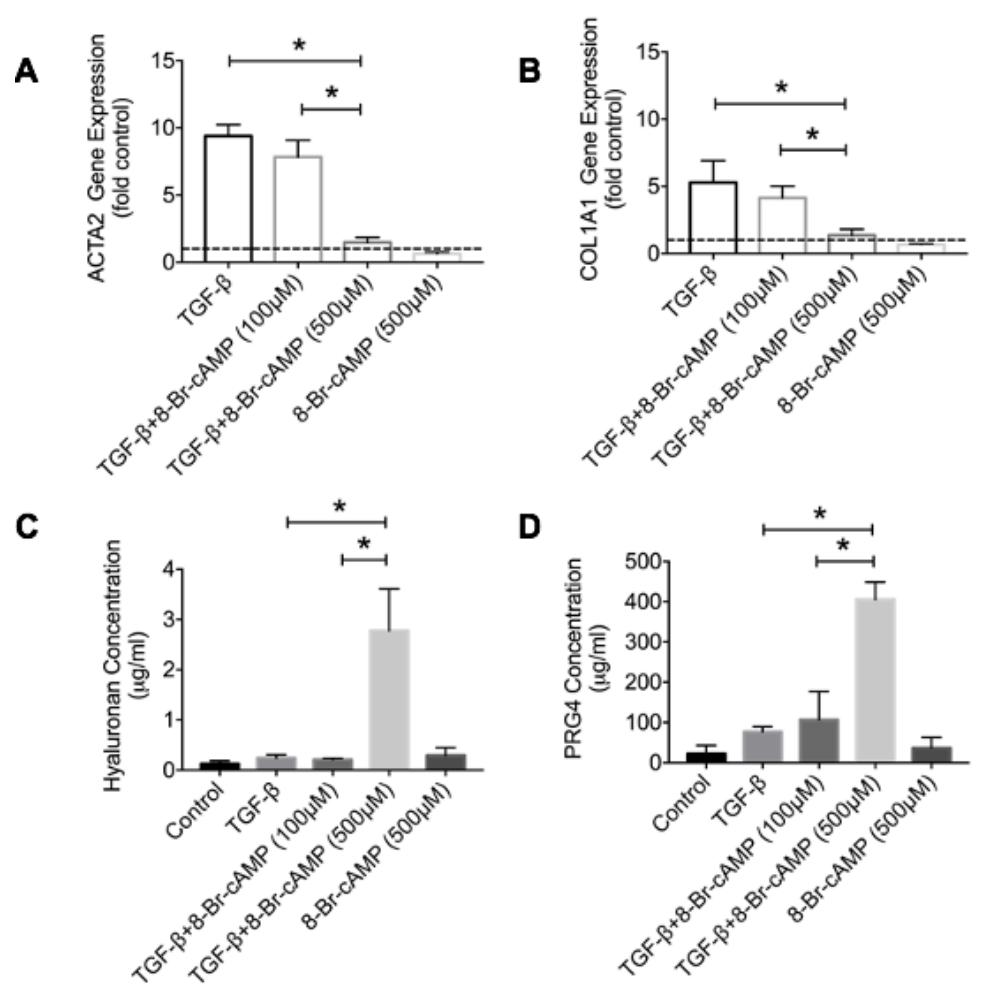

Fig. 3.4 Impact of 8-bromo cAMP (8-Br-cAMP; 100 and $500 \mu \mathrm{M})$ treatment on basal and transforming growth factor beta 1 (TGF- $\beta 1$ )-induced alpha smooth muscle actin (ACTA2) and collagen I (COL1A1) expression and hyaluronan (HA) and proteoglycan-4 (PRG4) secretion in osteoarthritic synoviocytes. Data is presented as the mean \pm S.D. of experiments utilizing OA synoviocytes from 3 patients. ${ }^{*} p<0.001$. A. 8 - $B r$-cAMP $(500 \mu \mathrm{M})$ reduced TGF- $\beta 1$ linked $A C T A 2$ expression in OA synoviocytes. B. 8-Br-cAMP $(500 \mu \mathrm{M})$ reduced TGF- $\beta 1$ linked COL1A1 expression in OA synoviocytes. C. 8-Br-cAMP $(500 \mu \mathrm{M})$ increased HA secretion in TGF$\beta 1$ stimulated OA synoviocytes. D. 8-Br-cAMP $(500 \mu \mathrm{M})$ increased PRG4 secretion in TGF$\beta 1$ stimulated OA synoviocytes. 
A
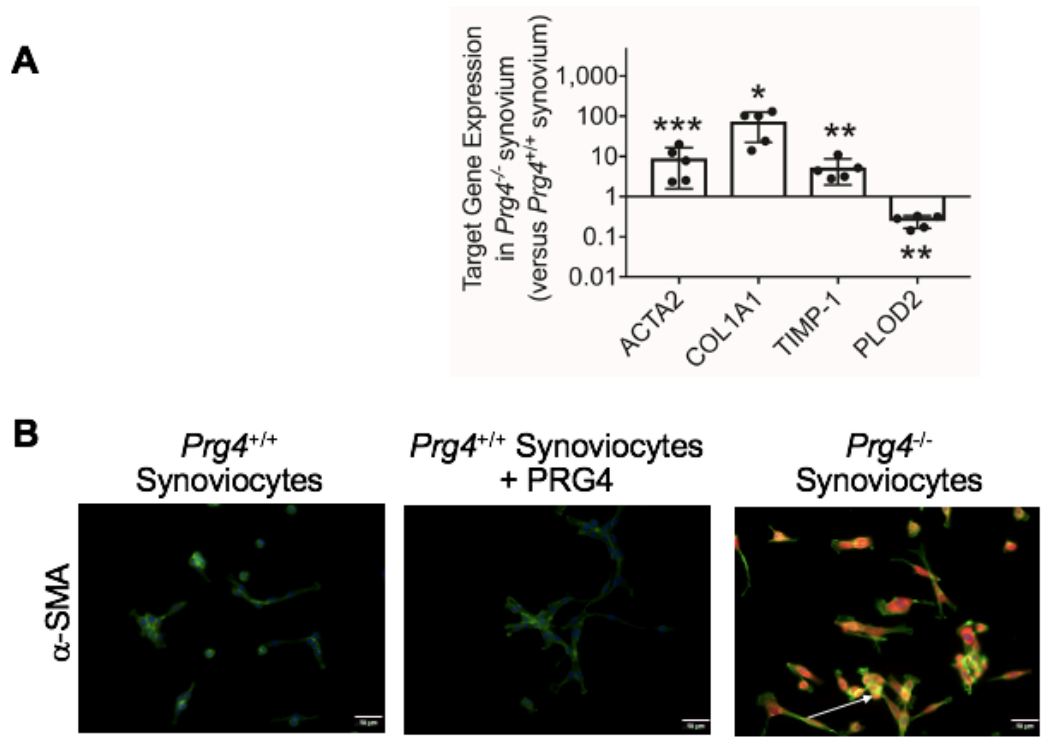

Prg $4^{-1-}$ Synoviocytes
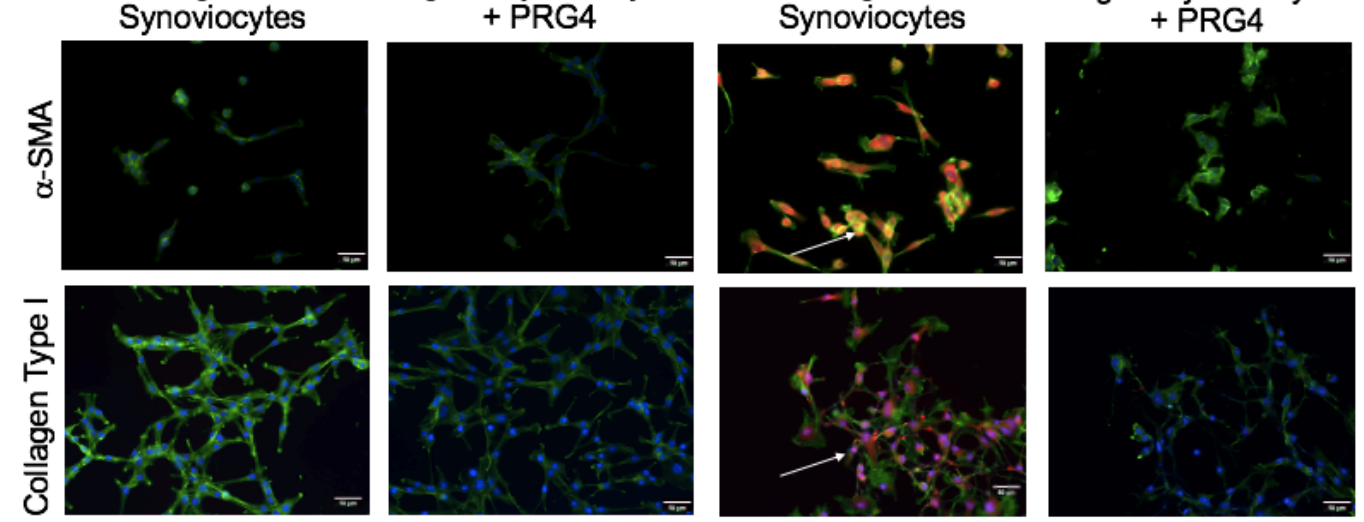

Fig. 3.5 Gene expression of alpha smooth muscle actin (ACTA2), collagen type I (COL1A1), tissue-inhibitor of metalloproteinase-1 (TIMP-1) and procollagen-lysine, 2-oxoglutarate 5dioxygenase 2 (PLOD2) in synovial tissues isolated from $\mathrm{Prg}^{+/+}$and $\mathrm{Prg}^{-/}$mice and immunocytostaining of alpha smooth muscle actin ( $\alpha$-SMA) and collagen type I in $\mathrm{Prg}^{+/+}$and $\operatorname{Prg}^{-/-}$synoviocytes and impact of human synoviocyte PRG4 treatment. ${ }^{*} p<0.001 ;{ }^{* *} p<0.01$; $* * * p<0.05$.

A. ACTA2, COL1A1 and TIMP-1 expression in $\operatorname{Prg}^{-/-}$synovial tissues was higher than $\operatorname{Prg}^{+/+}$ synovial tissues. PLOD2 expression in $\operatorname{Prg}^{4-/}$ synovial tissues was lower than $\operatorname{Prg}^{+/+}$synovial tissues. Each group contained 5 samples with each sample generated by pooling synovial tissues from 3 mice.

B. Merged images depicting $\alpha$-SMA and collagen type I protein immunostaining in isolated $\mathrm{Prg}^{+/+}$synoviocytes and $\mathrm{Prg}^{-/}{ }^{-/}$synoviocytes (bright orange) and counterstained with F-actin [198] and DAPI (blue). $\alpha$-SMA and collagen type I staining was detected in $\operatorname{Prg}^{4-/}$ synoviocytes (white arrows) and no staining was detected in $\operatorname{Prg}^{4^{+/+}}$synoviocytes. $\alpha$-SMA and collagen type I staining intensities were reduced by human synoviocyte PRG4 treatment for 24 hours. Scale $=$ $50 \mu \mathrm{m}$. 

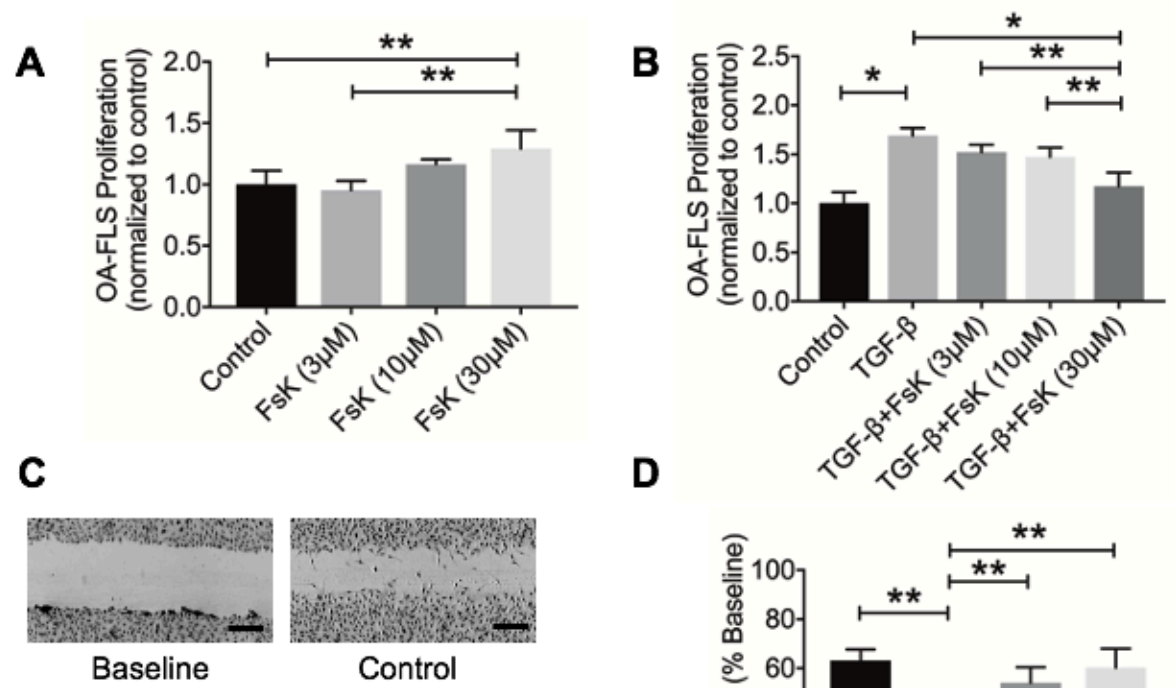

Baseline

Control
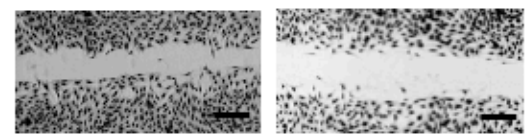

TGF- $\beta$ TGF- $\beta+F s K(10 \mu M)$
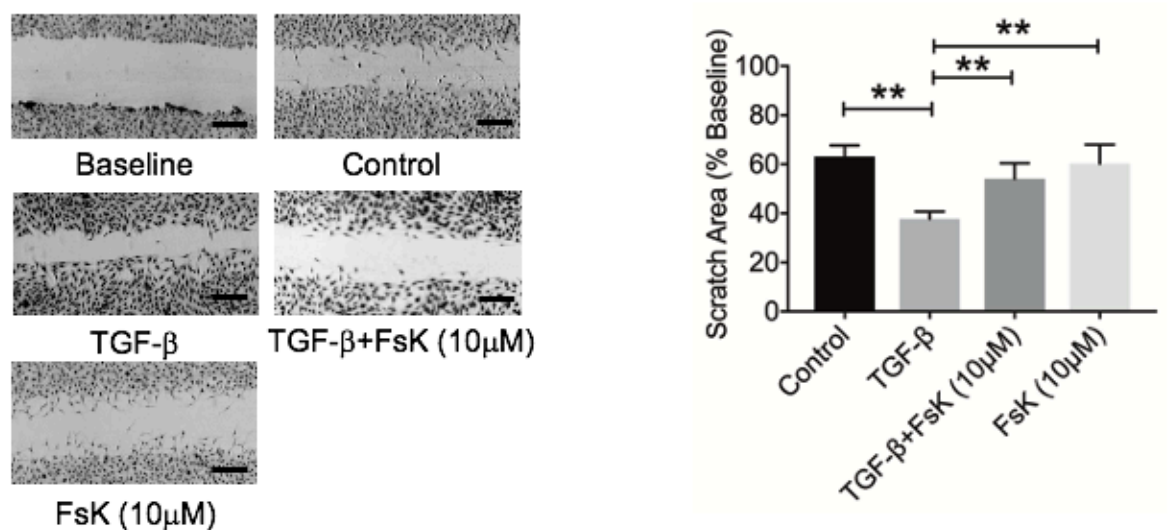

Fig. 3.6 Impact of forskolin (FsK) treatment on basal and transforming growth factor beta 1 (TGF$\beta 1)$ induced proliferation and migration of osteoarthritic (OA) synoviocytes. Data is presented as the mean \pm S.D. of experiments utilizing OA synoviocytes from 4 patients. ${ }^{*} p<0.001 ;{ }^{*} p<0.01$; $* * * p<0.05$. Scale $=1,000 \mu \mathrm{M}$. A. FsK $(30 \mu \mathrm{M})$ treatment enhanced basal OA synoviocyte proliferation. B. FsK $(30 \mu \mathrm{M})$ treatment reduced TGF- $\beta 1$-induced OA synoviocyte proliferation. C. Representative images showing baseline and 48-hour basal, TGF- $\beta 1$, TGF- $\beta 1+$ FsK and FsK alone induced $\mathrm{OA}$ synoviocyte migration using an in vitro scratch assay. treatment reduced TGF- $\beta 1$ stimulated OA synoviocyte migration. 


\section{CHAPTER 4}

Proteoglycan-4 Regulates Fibroblast to Myofibroblast Transition and Expression of Fibrotic Genes in the Synovium

Authors: Marwa Qadri, Gregory D. Jay, Ling X. Zhang, Holly Richendrfer, Tannin A. Schmidt, Khaled A. Elsaid

The following chapter was published in Arthritis Research \& Therapy (2020) 22:113 https://doi.org/10.1186/s13075-020-02207-x 
ABSTRACT

Background: Synovial tissue fibrosis is common in advanced OA with features including presence of stress fiber-positive myofibroblasts and deposition of cross-linked collagen type-I. An antifibrotic effect in OA synoviocytes was associated with PRG4 secretion and native synovial PRG4 reduced collagen content in OA synoviocytes. PRG4 is a ligand of the CD44 receptor. Our objective was to examine the role of PRG4-CD44 interaction in regulating synovial tissue fibrosis in vitro and in vivo.

Methods: OA synoviocytes were treated with TGF- $\beta \pm$ PRG4 for 24 hours and $\alpha$-SMA content was determined using immunofluorescence. Rhodamine labeled rhPRG4 was incubated with OA synoviocytes \pm anti-CD44 or isotype control antibodies and cellular uptake of rhPRG4 was determined following a 30-min incubation and $\alpha$-SMA expression following a 24-hour incubation. HEK-TGF- $\beta$ cells were treated with TGF- $\beta \pm$ rhPRG4 and Smad3 phosphorylation was determined using immunofluorescence and TGF- $\beta /$ Smad pathway activation was determined colorimetrically. We probed for stress fibers and focal adhesions (FAs) in TGF- $\beta$ treated murine fibroblasts and fibroblast migration was quantified \pm rhPRG4. Synovial expression of fibrotic markers: $\alpha$-SMA, collagen type-I and PLOD2 in Prg4 gene trap $\left(\operatorname{Prg} 4^{G T}\right)$ and recombined Prg $4^{G T R}$ animals was studied at 2 and 9 months of age. Synovial expression of $\alpha$-SMA and PLOD2 was determined in 2-months old $\operatorname{Prg} 4^{G T / G T} \& C d 44^{-/-}$and $\operatorname{Prg} 4^{G T R / G T R} \& C d 44^{-/-}$animals.

Results: PRG4 reduced $\alpha$-SMA content in OA synoviocytes $(p<0.001)$. rhPRG4 was internalized by OA synoviocytes via CD44 and CD44 neutralization attenuated rhPRG4's antifibrotic effect $(p<0.05)$. rhPRG4 reduced pSmad3 signal in HEK-TGF- $\beta$ cells $(p<0.001)$ and TGF- $\beta /$ Smad 
pathway activation $(p<0.001)$. rhPRG4 reduced the number of stress fiber-positive myofibroblasts, FAs mean size, and cell migration in TGF- $\beta$ treated NIH3T3 fibroblasts $(p<0.05)$. rhPRG4 inhibited fibroblast migration in a macrophage and fibroblast co-culture model without altering active or total TGF- $\beta$ levels. Synovial tissues of 9-months old $\operatorname{Prg} 4^{G T / G T}$ animals had higher $\alpha$-SMA, collagen type-I and PLOD2 $(p<0.001)$ content and Prg4 re-expression reduced these markers $(p<0.01)$. Prg4 re-expression also reduced $\alpha$-SMA and PLOD2 in CD44-deficient mice.

Conclusion: PRG4 is an endogenous antifibrotic modulator in the joint and its effect on myofibroblast formation is mediated by CD44, but CD44 is not required to demonstrate an antifibrotic effect in vivo.

Keywords: Proteoglycan-4, CD44, SMA, synovial fibrosis, myofibroblast 


\subsection{INTRODUCTION}

Osteoarthritis $(\mathrm{OA})$ is the most prevalent type of musculoskeletal disorder in patients 60 years or older [199-201]. OA is a leading cause of pain and disability and patients with symptomatic knee OA are at an increased risk of all-cause mortality [202-204]. The etiology of OA is complex where joint trauma, obesity and genetics, among other risk factors, contribute to disease development [205-208]. OA affects the whole joint with manifestations that include degeneration of articular cartilage and the meniscus, abnormal bone remodeling, and synovial inflammation [160, 209]. Macroscopic evidence of synovial inflammation or synovitis is a common finding in up to $74 \%$ of patients with knee OA of different grades and $95 \%$ of patients with moderate to severe OA [112, $210,211]$. OA synovitis is likely caused by an innate immune response, mediated by the toll-like receptors 2 and 4 (TLR2 and TLR4) in the synovium, and resultant expression of inflammatory cytokines, chemokines and matrix degrading enzymes [140, 212-215]. The extent of synovitis is a strong predictor of OA progression across multiple studies, and therefore treating synovial inflammation is a potentially important target for therapeutic intervention, especially during the early stage of OA $[112,157,216]$.

The normal synovium is composed of a cellular intimal layer and a subintimal connective tissue layer $[112,115]$. The intimal layer is usually $1-4$ cells in thickness with two types of cells; type A macrophages and type B synovial fibroblasts, with macrophages making up a smaller percentage of cells in the intimal layer of the healthy synovium [115]. Synovial fibroblasts synthesize hyaluronic acid (HA), a glycosaminoglycan, and proteoglycan-4 (PRG4), a mucinous glycoprotein, which are major components of synovial fluid (SF) with important roles in joint lubrication $[195,217]$. In synovitis, synovial membranes become hyperplastic with increased accumulation of macrophages and other inflammatory cells, along with subintimal fibrosis and 
angiogenesis $[112,160,161]$. Synovial fibrosis can be considered a maladaptive healing response to chronic synovitis which is characterized by upregulation of collagen type-I expression, fibroblast transition to a myofibroblast-like phenotype through de-novo expression of alpha smooth muscle actin ( $\alpha$-SMA) and enhanced migratory behavior, and upregulation of procollagenlysine, 2-oxogluterate 5-dioxygenase 2 (PLOD2) leading to accumulation of cross-linked collagen in the synovium $[164,218]$. These pathological changes are induced in the OA synovium by transforming growth factor beta (TGF)- $\beta 1$ and its associated signaling pathways [162, 183]. Synovial fibrosis independently contributes to joint pain and stiffness observed in advanced OA $[161,219]$.

In a recent study, we demonstrated that increasing intracellular adenosine $3^{\prime}, 5^{\prime}$-cyclic monophosphate (cAMP), using forskolin, reduced collagen type-I, PLOD2 and $\alpha$-SMA expression in TGF- $\beta$ stimulated osteoarthritic fibroblast-like synoviocytes (OA FLS) and reduced fibroblast to myofibroblast transition, as evidenced by blocking stress fiber formation [220]. Associated with this antifibrotic effect was an increase in HA and PRG4 production by OA FLS, whereby native synovial human PRG4 reduced procollagen type-I level in OA FLS [220]. Furthermore, synoviocytes from Prg4 null mice displayed more extensive collagen type-I and $\alpha$-SMA immunostaining compared to synoviocytes from Prg4 competent mice [220]. In a separate study, we have also shown that PRG4 is a ligand for the HA receptor, CD44 [154]. We have also reported that PRG4-CD44 interaction inhibited interlukin-1 beta (IL-1 13$)$ induced OA FLS proliferation and expression of matrix degrading enzymes [155], via the inhibition of nuclear factor kappa $b$ (NF$\kappa \mathrm{B})$ nuclear translocation mediated by blocking inhibitory kappa b (IкB) degradation [155]. 
It remains unknown whether PRG4 has a role in regulating fibroblast to myofibroblast transition and associated cell migration in the fibrotic synovium. Furthermore, it is yet to be determined whether PRG4 regulates synovial fibrosis in vivo and whether this role is due to its interaction with the CD44 receptor. Using recombinant human proteoglycan-4 (rhPRG4),we aimed to study the role of PRG4 in regulating fibroblast to myofibroblast transition and modulating fibroblast migration in response to exogenous TGF- $\beta$ or co-incubation with lipopolysaccharide (LPS) stimulated macrophages. We also studied the role of PRG4-CD44 interaction, and more specifically CD44-mediated cellular uptake of PRG4, in the regulation of myofibroblast formation in vitro and progression of synovial fibrosis in vivo. We hypothesized that PRG4 regulates fibroblast to myofibroblast transition and prevents synovial fibrosis in a CD44-dependent manner.

\subsection{METHODS}

4.2.1 Impact of PRG4 and HA treatments on ACTA2 expression, $\alpha$-SMA immunostaining, and stress fiber formation in osteoarthritic fibroblast-like synoviocytes (OA FLS) and role of CD44 in mediating the effect of rhPRG4 in TGF- $\beta$ stimulated OA FLS

OA FLS (Cell Applications, USA) were isolated from synovial tissues from de-identified patients undergoing knee replacement surgery ( $n=4 ; 2$ males and 2 females; $62-69$ years old). Cells were received in their second passage and were cultured as previously described [213]. OA FLS were used between the $3^{\text {rd }}$ and $6^{\text {th }}$ passages to avoid alterations in gene expression pattern and cell proliferation rate [176]. OA FLS $(300,000$ cells/well) in serum-free DMEM media were treated with recombinant human TGF- $\beta 1$ (R\&D Systems, USA) $(1 \mathrm{ng} / \mathrm{mL}) \pm$ native human synovial PRG4 (apparent mol mass $280 \mathrm{kDa}$ as a monomer; $100 \mu \mathrm{g} / \mathrm{mL}$ ) [179] or high molecular weight hyaluronic acid (HA; 1,200 kDa; R\&D Systems; $100 \mu \mathrm{g} / \mathrm{mL}$ ) for 24 hours. RNA isolation, cDNA synthesis and quantitative PCR were performed as previously described [220]. The cycle threshold (Ct) value of $\alpha$-SMA (ACTA2) was normalized to the Ct value of GAPDH in the same sample, and the 
relative expression was calculated using the $2^{-\Delta \Delta \mathrm{Ct}}$ method [134]. All primers and probes utilized in our study are commercially available (Thermo Fisher Scientific, USA).

Assessment of $\alpha$-SMA content in OA FLS was conducted using immunofluorescence and determination of corrected total cell fluorescence (CTCF) using a Nikon E600 fluorescence microscope. OA FLS (200,000 cells/well) were cultured on collagen type I-coated $22 \mathrm{~mm}$ glass coverslips for 48 hours in DMEM medium $+10 \%$ fetal bovine serum (FBS). Cells were treated with TGF- $\beta 1(1 \mathrm{ng} / \mathrm{mL}) \pm$ PRG4 or HA $(100 \mu \mathrm{g} / \mathrm{mL}$ for both treatments) for 48 hours in serum-free DMEM medium. Subsequently, cells were fixed in $10 \%$ neutral buffered formalin for $15 \mathrm{~min}$ and washed twice with phosphate buffered saline (PBS). Cells were permeabilized using $0.01 \%$ Triton X-100 in PBS and blocked using 2\% bovine serum albumin (BSA; Sigma Aldrich, USA) in PBS for 2 hours at room temperature. Probing for $\alpha$-SMA was performed using FITC-conjugated anti$\alpha$-SMA antibody (1:100 dilution; Abcam, USA) and counterstained using Alexa Fluor 594conjugated anti- $\alpha$-tubulin antibody (1:100 dilution: Abcam) overnight at $4{ }^{\circ} \mathrm{C}$. Following washing with PBS, cells were mounted on DAPI mounting shield (Abcam) and CTCF was quantified using 4 different fields per slide and mean CTCF was calculated. Presence of stress fibers in OA FLS was also evaluated.

Recombinant human PRG4 (rhPRG4; apparent mol mass $240 \mathrm{kDa}$ ) is an endotoxin-free fulllength product produced by CHO-M cells (Lubris, Framingham, USA) [221]. Rhodamine labeling of rhPRG4 was performed using the Pierce NHS-Rhodamine Antibody Labeling Kit (Thermo Fisher Scientific). OA FLS (200,000 cells/well) were cultured on collagen type-I coated cover slips and incubated with rhodamine-rhPRG4 $(25 \mu \mathrm{g} / \mathrm{mL}) \pm$ anti-CD44 or isotype control (IC) antibodies $(2 \mu \mathrm{g} / \mathrm{mL}$ for both antibodies; Abcam) for 30 minutes. Cells were pre-incubated with 
the antibodies for 1-hour prior to rhPRG4 addition. Subsequently, cells were washed twice with PBS and mounted on DAPI mounting media and CTCF was quantified as described above. In another set of experiments, OA FLS $(300,000$ cells/well) were treated with TGF- $\beta 1(1 \mathrm{ng} / \mathrm{mL}) \pm$ rhPRG4 $(100 \mu \mathrm{g} / \mathrm{mL}) \pm$ anti-CD44 or IC antibodies $(2 \mu \mathrm{g} / \mathrm{mL}$ for both antibodies $)$ for 24 hours followed by determination of ACTA2 expression as described above.

\subsubsection{CD44-dependent uptake of rhPRG4, Smad3 phosphorylation and regulation of TGF- $\beta /$ Smad pathway activation in TGF- $\beta$ stimulated HEK Blue-TGF- $\beta$ cells}

HEK Blue-TGF- $\beta$ is an engineered reporter cell line produced by transfecting human embryonic kidney (HEK) cells with TGF- $\beta$ receptor 1 (TGF- $\beta$ R1), Smad3 and Smad4 genes (Invivogen, USA). TGF- $\beta 1$-treatment results in phosphorylation of Smad3 (pSmad3) and Smad4 (pSmad4), translocation to the nucleus and expression of secreted alkaline phosphatase (SEAP). The activity of SEAP can be detected colorimetrically in media supernatants using a specific substrate (Quanti Blue) at 620-655 nm (Invivogen). HEK-TGF- $\beta$ cells $(500,000)$ were plated onto sterile chamber slides and incubated with rhodamine-rhPRG4 $(25 \mu \mathrm{g} / \mathrm{mL})$ for 1 hour \pm anti CD44 or IC antibodies $(2 \mu \mathrm{g} / \mathrm{mL}$ for both antibodies). Cells were pre-incubated with the antibodies for 1-hour prior to rhPRG4 addition. Subsequently, cells were washed with PBS and intracellular CTCF was determined as described above. In another set of experiments, rhodamine labeled rhPRG4 $(25 \mu \mathrm{g} / \mathrm{mL})$ was incubated with HEK-TGF- $\beta$ cells and CD44 was probed using FITC-conjugated anti-CD44 antibody (Abcam) and CD44 [198] and rhPRG4 (red) co-localization was examined using a confocal microscope.

Immunostaining of pSmad3 was performed using an anti-Smad3 antibody that detects phosphorylated serine residues $\left(1: 1,000\right.$ dilution overnight at $4{ }^{\circ} \mathrm{C}$; Abcam). HEK-TGF- $\beta$ cells were stimulated with TGF- $\beta 1 \pm$ rhPRG4 $(150 \mu \mathrm{g} / \mathrm{mL})$ for 1 hour. Subsequently, cells were fixed, 
washed with PBS and permeabilized as described above. Following probing with anti-pSmad3 antibody, cells were washed with PBS and incubated with goat anti-rabbit IgG (Alexa Fluor 488) (1:1,000 dilution for 1 hour at room temperature; Abcam). Cells were subsequently washed with PBS and green immunofluorescence was quantified across all experimental groups. To investigate the impact of rhPRG4 on TGF- $\beta /$ Smad pathway activation, HEK-TGF- $\beta$ cells $(100,000$ cells/well) were cultured in sterile 96 well plates \pm TGF- $\beta 1(1 \mathrm{ng} / \mathrm{mL}) \pm \operatorname{rhPRG} 4(50,100$ or $150 \mu \mathrm{g} / \mathrm{mL})$ for 24 hours in Quanti-Blue media and the $655 \mathrm{~nm}$ absorbance intensity was determined. Data are presented as fold absorbance intensities across experimental groups normalized to untreated controls.

4.2.3 TGF- $\beta$ induction of stress fibers, focal adhesions (FAs) and cell migration in murine NIH3T3 fibroblasts, comparison of stress fibers and FAs in $\mathrm{Prg4}^{+/+}$and Prg4 ${ }^{-/-}$synovial fibroblasts and role of rhPRG4 in regulating fibroblast migration

Murine fibroblasts (NIH3T3; ATCC; 20,000 cells/well) were cultured on collagen type I-coated 22-mm glass coverslips for 24 hours in DMEM supplemented with $10 \%$ bovine calf serum (BCS). NIH3T3 cells were starved in DMEM containing 1\% BCS for 24 hours. Murine fibroblasts were treated with murine TGF- $\beta 1(1 \mathrm{ng} / \mathrm{ml}) \pm$ rhPRG4 $(200 \mu \mathrm{g} / \mathrm{mL})$ for 24 hours in serum-free DMEM. Subsequently, fibroblasts were fixed using neutral buffered formalin, washed with PBS, permeabilized using $0.1 \%$ triton $\mathrm{X}-100$ and probed using an anti- $\alpha$-SMA (marker of stress fibers; 1:1,000) or anti-vinculin (marker of FA complex; 1:1,000) overnight at $4^{\circ} \mathrm{C}$. After washing with PBS, cells were incubated with goat anti rabbit IgG (Alexa Fluor ${ }^{\circledR} 488$ ) at 1:1,000 dilution for 1 hour at $4^{\circ} \mathrm{C}$. Rhodamine-labeled phalloidin (cytoskeleton label; 1:1,000) was added with the secondary antibody. All antibodies were obtained from Abcam. Cells were washed and mounted with DAPI medium for 2 hours and viewed under a confocal microscope. The number of stress fiber-positive fibroblasts was determined by a blinded investigator. At least 100 cells over at least 
5 different fields were evaluated, and the percentage of stress-fiber positive fibroblasts was calculated. Separately, mean fibroblast cell spread area was determined using NIS-Elements imaging software (Nikon). The number and mean size of FAs per cell were determined using ImageJ software as previously described [222]. Fibroblast migration was determined using a scratch assay. A $1,000 \mu \mathrm{L}$ pipette tip was used to perform a uniform scratch in the confluent NIH3T3 fibroblast monolayer. TGF- $\beta(1 \mathrm{ng} / \mathrm{mL})$ stimulation was performed for 48 hours \pm rhPRG4 $(200 \mu \mathrm{g} / \mathrm{mL})$. Subsequently, cells were washed and stained (Cell Biolabs) and imaged using an all-in-one fluorescence microscope (Keyence). A region of interest was defined and scratch widths were determined across multiple locations. Cell migration was expressed as percent wound closure across different experimental groups as previously described [223].

The phenotype of $\operatorname{Prg} 4^{-/}$mice is characterized by synovial membrane hyperplasia, chondrocyte apoptosis, and cartilage surface fibrillation [180]. The $\operatorname{Prg} 4^{-/}$mouse colony is maintained by Dr. Gregory Jay at Rhode Island Hospital (RIH) and is commercially available (stock no. 025737; JAX, USA). All animal experiments were approved by the IACUC committee at RIH and all experiments were performed according to all applicable guidelines and regulations. Synoviocyte isolation from $\operatorname{Prg} 4^{-/}$and $\operatorname{Prg}^{+/+}$synovial tissues was performed as previously described [154, 220]. $\operatorname{Prg}^{-/-}$and $\operatorname{Prg}^{+/+}$synoviocytes were plated onto sterile chamber slides (Thermo Fisher Scientific) and allowed to adhere for 48 hours. Subsequently, cells were stained for $\alpha$-SMA and vinculin as described above. The mean size of FAs per cell in $\operatorname{Prg} 4^{-/-}$and $\operatorname{Prg} 4^{+/+}$synoviocytes was determined as described above. $\operatorname{Prg} 4^{-/}$and $\operatorname{Prg} 4^{+/+}$synoviocytes were seeded in 6 well plates and a scratch was performed in the confluent cell monolayer as described above. Basal synovial fibroblast migration of both genotypes was quantified over 48 hours \pm rhPRG4 $(200 \mu \mathrm{g} / \mathrm{mL})$, antiCD44 $(2 \mu \mathrm{g} / \mathrm{mL})$ or IC $(2 \mu \mathrm{g} / \mathrm{mL})$ antibodies as previously described [223]. 
4.2.4 Generation of Active TGF- $\beta$ in lipopolysaccharide-stimulated murine macrophage J774A and NIH3T3 fibroblast co-culture and impact of rhPRG4 treatment on fibroblast migration

Murine J774A macrophages (ATCC) were cultured in DMEM medium + 10\% FBS. Macrophages $(300,000$ cells in DMEM medium + 10\% FBS) were stimulated with lipopolysaccharide (LPS; Invivogen) $(5 \mu \mathrm{g} / \mathrm{mL})$ for 24 hours. Subsequently, murine macrophages were washed five times with DMEM medium and transferred to the top chamber of a transwell co-culture system $(0.4 \mu \mathrm{m}$ pore size; Sigma Aldrich). Murine NIH3T3 cells were seeded in the lower chamber of the transwell system and a scratch was performed as described above. NIH3T3 migration was determined following a 48-hour incubation of fibroblasts with macrophages in the co-culture system as described above \pm rhPRG4 $(200 \mu \mathrm{g} / \mathrm{mL})$. Active and total TGF- $\beta$ media levels were determined using an ELISA (R\&D Systems).

\subsubsection{Age-dependent expression of fibrotic markers in synovial tissues from Prg4 GT/GT animals and the role of PRG4/CD44 interaction in modulating synovial fibrosis in vivo}

The Prg4 gene-trap (Prg $4^{G T}$ ) mouse colony is maintained by Dr. Gregory Jay at RIH and is commercially available (stock no. 025740; JAX) [224]. Prg4 $4^{G T}$ animal is a genetically engineered PRG4-deficient mouse where the Prg4 expression can be restored via CRE-mediated recombination [224]. The $\operatorname{Prg} 4^{G T / G T}$ mouse recapitulates the hallmark findings in $\operatorname{Prg} 4^{-/-}$mouse, namely synovial tissue hyperplasia and cartilage surface fibrillations and recombination in 3weeks old animals improved but did not completely normalize joint pathological findings [224]. In our studies, recombination $\left(\operatorname{Prg} 4^{G T R / G T R}\right)$ occurred in 3-weeks old animals via intraperitoneal injection of tamoxifen $(0.1 \mathrm{mg} / \mathrm{g}$ in $100 \mu \mathrm{L}$ corn oil vehicle) daily for 10 days. We compared gene expression and immunostaining of fibrotic markers: $\alpha$-SMA, COL1A1 (collagen type-I) and PLOD2 in 2-months old Prg4 ${ }^{G T / G T}$, 2-months old Prg4 ${ }^{G T R / G T R}$, 9-months old Prg4 ${ }^{G T / G T}$ and 9- 
months old Prg4 ${ }^{G T R / G T R}$ animals. ACTA2, COL1A1 and PLOD2 expression levels in murine synovial tissues were performed as previously described [220]. Tissues from each three consecutive mice were pooled and underwent RNA isolation, generating five pooled samples in each experimental group. Separately, animal joints ( $n=5$ in each group) underwent decalcification and paraffin-embedded sectioning as previously described [224]. Immunostaining of synovial tissues was performed using primary antibodies against $\alpha$-SMA, COL1A1 or PLOD2 (1:1,000 dilutions performed overnight at $4^{\circ} \mathrm{C}$ ) (All antibodies were purchased from Abcam). Subsequently, sections were washed and incubated with goat anti-rabbit IgG (Alexa Fluor 488) (1:1,000 dilution) for 1 hour and fluorescence intensities (expressed as lumens $/ \mathrm{mm}^{2}$ ) were quantified using a fluorescence microscope.

To appreciate the significance of PRG4 and CD44 interaction in the context of fibrotic markers' expression in the synovium, $\operatorname{Prg} 4^{G T / G T}$ animals were crossed with $C d 44^{-/-}$mice (stock no. 005085; JAX) [135] to generate $C d 44^{-/} \& \operatorname{Prg} 4^{G T / G T}$ animals. Recombination occurred in 3-weeks old animals to generate $C d 44^{-/} \& \operatorname{Prg} 4^{G T R / G T R}$ animals as described in the last paragraph. Histological analyses of joints harvested from 2-months old animals (at least 4 animals per group) were performed as described above, using $C d 44^{+/+} \& \operatorname{Prg} 4^{G T / G T}$ animals from the same litters as controls. We probed synovial tissues for the following fibrotic markers: $\alpha$-SMA and PLOD2 using specific primary antibodies (1:1,000 dilutions) followed by goat anti-rabbit IgG (Alexa Fluor 488) and quantitation of fluorescence intensities (expressed as lumens $/ \mathrm{mm}^{2}$ ) as described above. 


\subsection{Statistical Analyses}

Target gene expression was statistically evaluated by comparing $\Delta \mathrm{C}_{t}\left(\mathrm{C}_{\mathrm{t}}\right.$ value of target gene- $\mathrm{C}_{\mathrm{t}}$ value of GAPDH in the same sample) values of different experimental groups. Statistical significance comparing two groups or multiple groups with parametric data was assessed by Student's $t$ test or ANOVA followed by post-hoc multiple comparisons (Tukey's post-hoc test). Statistical significance comparing two groups or multiple groups with nonparametric data was assessed by Rank Sum test or ANOVA on the ranks. A $p$ value of $<0.05$ was considered statistically significant. Data are presented as scatter plots with mean and standard deviations highlighted.

\subsection{RESULTS}

4.4.1 PRG4 reduced ACTA2 expression and stress fiber formation in osteoarthritic fibroblast-like synoviocytes (OA FLS) and CD44 was involved in the uptake of rhPRG4 by OA FLS, whereas rhPRG4-CD44 interaction affected ACTA2 expression in response to TGF- $\beta$

PRG4 and HA demonstrated equivalent efficacy in reducing ACTA2 expression in OA FLS (Fig.

4.1A). Both treatments reduced ACTA2 expression compared to the positive control TGF- $\beta 1$ $(p<0.001$ for TGF- $\beta 1+$ PRG4 or TGF- $\beta 1+$ HA against TGF- $\beta 1$ alone). In addition to reducing ACTA2 expression, PRG4 reduced $\alpha-S M A$ content in OA FLS $(p<0.001$ versus TGF- $\beta 1$ alone; Fig. 4.1C). A similar effect was also observed for HA treatment ( $p<0.001$ versus TGF- $\beta 1$ alone; Fig. 4.1C). The effect of PRG4 was biologically significant with approximately $52 \%$ reduction in mean $\alpha$-SMA immunofluorescence compared to TGF- $\beta 1$ alone. In addition to reducing $\alpha$-SMA in OA FLS, PRG4 and HA treatments prevented the formation of stress fibers in OA FLS in response to TGF- $\beta 1$ stimulation (Fig. 4.1B). rhPRG4 was rapidly internalized by OA FLS and the mechanism of its internalization was related to its interaction with CD44. This was illustrated by 
a reduction in OA FLS CTCF with an anti-CD44 treatment (Fig. 4.1D \& Fig. 4.1E). Neutralization of CD44 receptor did not completely block rhPRG4 uptake by OA FLS (Fig. 4.1E). Alternatively, treatment with an IC antibody did not alter rhPRG4's uptake by OA FLS ( $p>0.05$; Fig. 4.1E). Inhibition of $A C T A 2$ expression by rhPRG4 was reversed by pre-incubation with an anti-CD44 antibody $(p<0.05$ for TGF- $\beta 1+$ rhPRG4 + anti-CD44 versus TGF- $\beta 1+$ rhPRG4; Fig. 4.1F). In contrast, IC antibody treatment did not alter the magnitude of reduction in ACTA2 expression observed with rhPRG4 (Fig. 4.1F).

\subsubsection{CD44 receptor facilitated rhPRG4 uptake by HEK-TGFß cells and rhPRG4 inhibited TGF-ß1/Smad pathway activation}

Representative images demonstrate intracellular red fluorescence following the incubation of rhodamine-labeled rhPRG4 with HEK-TGF $\beta$ cells (Fig. 4.2A). Antibody-mediated CD44 receptor neutralization reduced the intensity of red fluorescence, indicative of reduced rhPRG4 uptake into HEK-TGF $\beta$ cells $(p<0.001$ vs. rhPRG4 alone; Fig. 4.2A and Fig. 4.2B). This effect was specific to CD44 receptor, as a non-specific IC antibody pre-incubation did not alter the extent of rhPRG4 uptake into HEK-TGF $\beta$ cells ( $p>0.05$; Fig. 4.2A and Fig. 4.2B). Further evidence of CD44 involvement in rhPRG4 uptake by HEK-TGF $\beta$ cells is provided by the intracellular co-localization of rhPRG4 (red) and CD44 [198] as shown by arrows (yellow color) (Fig. 4.2A). TGF- $\beta 1$ stimulation resulted in Smad3 phosphorylation as shown in representative images (Fig. 4.2C). Intense green fluorescence in TGF- $\beta 1$-stimulated HEK-TGF- $\beta$ cells indicated the formation of pSmad3. rhPRG4 treatment reduced mean pSmad3 staining intensity subsequent to TGF- $\beta 1$ stimulation ( $p<0.001$; Fig. 4.2D). In addition, rhPRG4 treatment dose-dependently reduced TGF$\beta 1 /$ Smad pathway activation as shown by a reduction in the formation of SEAP colored product $(p<0.05$ for 100 and $150 \mu \mathrm{g} / \mathrm{mL}$ concentrations versus TGF- $\beta 1$ alone; Fig. $4.2 \mathrm{E})$. 
4.4.3 TGF- $\beta$ induced stress fiber and FAs formation in murine fibroblast NIH3T3 cells resulting in enhanced cell migration, and these effects were reduced with rhPRG4 treatment

Representative images highlight that murine TGF- $\beta$ induced the formation of stress fibers and vinculin expression and thus FAs formation in NIH3T3 cells (Fig. 4.3A). TGF- $\beta$ increased the percentage of stress fiber-positive NIH3T3 cells by $\sim 5$-folds $(p<0.001$ against control cells; Fig. 4.3B), and increased mean cell spread area by $\sim 2$-folds ( $p<0.001$ against control cells; Fig. 4.3C). rhPRG4 treatment reduced the percentage of stress fiber-positive NIH3T3 cells $(p<0.001$; Fig. 4.3B) and mean cell spread area $(p<0.001$; Fig. $4.3 \mathrm{C})$ to control levels. TGF- $\beta$ increased the number of FAs in NIH3T3 cells by $\sim 4$-folds ( $p<0.001$; Fig. 4.3D), and the mean size of FAs per cell by $\sim 2$-folds $(p<0.05$; Fig. 4.3E). Likewise, rhPRG4 treatment reduced the number of FAs $(p<0.001$; Fig. 4.3D) and mean FA size $(p<0.05$; Fig. 4.3E) to control levels. A functional outcome of TGF- $\beta$ induced stress fiber and FAs formation was the enhanced migration of NIH3T3 fibroblasts (Fig. 4.3F). rhPRG4 reduced TGF- $\beta$ stimulated NIH3T3 fibroblast migration $(p<0.001$; Fig. 4.3G) while rhPRG4 alone did not change basal NIH3T3 fibroblast migration ( $p>0.05$ against control; Fig. 4.3G).

\subsubsection{Lack of Prg4 expression in murine synovial fibroblasts was associated with enhanced stress fiber formation, FA size and enhanced basal cell migration which was reduced by rhPRG4 or anti-CD44 antibody treatments}

Representative images demonstrate enhanced $\alpha$-SMA and vinculin staining in $\mathrm{Prg}^{-/-}$synoviocytes compared to $\operatorname{Prg}^{+/+}$synoviocytes (Fig. 4.4A). The mean size of FAs was $\sim 2$-fold higher in $\operatorname{Prg} 4^{-}$ /- synoviocytes compared to $\operatorname{Prg}^{+/+}$synoviocytes $\left(p<0.001\right.$; Fig. 4.4B). Prg $4^{-/-}$synoviocytes displayed enhanced basal migration compared to $\operatorname{Prg} 4^{+/+}$synoviocytes $(p<0.01$; Fig. $4.4 \mathrm{C}$ and Fig. 4.4D). rhPRG4 and anti-CD44 antibody treatments reduced $\mathrm{Prg}^{-/-}$synoviocyte migration $(p<0.01$ for both treatments; Fig. 4.4D) with no significant difference between both treatments $(p>0.05)$. 
4.4.5 LPS stimulation of macrophages increased active and total TGF- $\beta$ levels in a macrophage and fibroblast co-culture model, induced fibroblast migration and rhPRG4 treatment reduced fibroblast migration without altering active or total TGF- $\beta$ levels

In response to LPS stimulation and co-culture of macrophages (M) and fibroblasts (F), active TGF$\beta$ levels in the macrophage and fibroblast co-culture were higher compared to fibroblasts alone $(p<0.001$; Fig. 4.5A). Correspondingly, total TGF- $\beta$ levels in the macrophage and fibroblast coculture were higher compared to fibroblasts alone $(p<0.001$; Fig. $4.5 \mathrm{~A})$. The increase in active and total TGF- $\beta$ levels resulted in enhanced fibroblast migration in the macrophage and fibroblast co-culture compared to fibroblasts alone ( $p<0.001$; Fig. $4.5 \mathrm{~B}$ and Fig. $4.5 \mathrm{C})$. rhPRG4 treatment reduced fibroblast migration in the co-culture model ( $p<0.001$; Fig. $4.5 \mathrm{C})$. The effect of rhPRG4 was not mediated by an alteration in active or total TGF- $\beta$ levels, shown by the lack of differences in active or total TGF- $\beta$ concentrations between rhPRG4-treated and untreated cells $(p>0.05$; Fig. 4.5D).

4.4.6 Lack of Prg4 expression resulted in progressive synovial tissue fibrosis, shown by enhanced $\alpha$-SMA, collagen type-I and PLOD2 production and Prg4 re-expression reduced synovial tissue fibrosis and this effect was evident in CD44-deficient mice

Synovial tissues from Prg $4^{G T / G T}$ animals displayed higher ACTA2, COL1A1 and PLOD2 expression levels compared to Prg4 wildtype synovia $(p<0.001$ for all comparisons; Fig. 4.6A, Fig. 4.6D and Fig. 4.6G). Interestingly, lack of Prg4 expression resulted in a greater increase in ACTA2 expression compared to COL1A1 and PLOD2 ( $p<0.001$ for all comparisons). The expression of fibrotic markers in synovial tissues from $\operatorname{Prg} 4^{G T / G T}$ animals progressed with age. ACTA2, COL1A1 and PLOD2 expression levels in 9 months-old Prg $4^{G T / G T}$ animals were higher than corresponding expression levels in 2 months-old $\operatorname{Prg} 4^{G T / G T}$ animals $(p<0.001$ for all comparisons). The regulation of fibrotic gene expression in the synovium by PRG4 was further confirmed by the reduction in ACTA2, COL1A1 and PLOD2 expression in 9 months-old 
$\operatorname{Prg} 4^{G T R / G T R}$ animals compared to age-matched $\operatorname{Prg} 4^{G T / G T}$ animals ( $p<0.001$ for all comparisons). Representative images of synovial tissues stained for $\alpha$-SMA, collagen type-I and PLOD2 proteins are shown in Figures 4.6B, 4.6E and 4.6H. There was no significant difference in mean collagen type-I staining intensity between 2 months-old $\operatorname{Prg} 4^{G T / G T}$ and age-matched $\operatorname{Prg} 4^{G T R / G T R}$ animals ( $p>0.05$; Fig. 4.6F). Alternatively, mean $\alpha$-SMA, collagen type-I and PLOD2 staining intensities were lower in 9 months-old $\operatorname{Prg} 4^{G T R / G T R}$ animals compared to age-matched $\operatorname{Prg} 4^{G T / G T}$ animals $(p<0.05$ for all comparisons; Fig. 4.6C, Fig. 4.6F and Fig. 4.6I).

The contribution of CD44 to PRG4's effect on $\alpha$-SMA and PLOD2 expression was further studied in vivo by comparing histological staining intensities in synovial tissues from $C d 44^{+/+} \& \operatorname{Prg} 4^{G T / G T}$, $C d 44^{-/} \& \operatorname{Prg} 4^{G T / G T}$, and $C d 44^{-} \& \operatorname{Prg} 4^{G T R / G T R}$ (Fig. 4.7). While mean $\alpha$-SMA staining intensity trended higher in $C d 44^{-/} \& \operatorname{Prg} 4^{G T / G T}$ synovia compared to $C d 4^{+/+} \& \operatorname{Prg} 4^{G T / G T}$ synovia, this increase did not reach statistical significance $(p=0.16)$. Mean $\alpha$-SMA staining intensity in $\mathrm{Cd}_{4} 4^{-}$ ${ }^{-} \& \operatorname{Prg} 4^{G T R / G T R}$ synovia was lower than corresponding mean intensity in $C d 44^{-/} \& \operatorname{Prg} 4^{G T / G T}$ synovia $(p<0.05)$. The absence of CD44 receptor increased PLOD2 staining in synovial tissues as evidenced by a higher mean staining intensity in $C d 44^{-/} \& \operatorname{Prg} 4^{G T / G T}$ animals compared to $C d 44^{+/+} \& \operatorname{Prg} 4^{G T / G T}$ animals $(p<0.001)$. In addition, Prg4 re-expression reduced mean PLOD2 staining in otherwise $C d 44$ null animals $(p<0.001)$.

\subsection{DISCUSSION}

PRG4, also called lubricin and superficial zone protein, is a mucinous glycoprotein with a protein core of 1,404 amino acids and a central mucin domain that is extensively glycosylated with $\mathrm{O}-$ linked $\beta(1-3)$ Gal-GalNAc oligosaccharides $[195,217,225,226]$. PRG4 is secreted from synovial fibroblasts and superficial zone chondrocytes and is found adsorbed on articular surfaces and the 
synovial membrane in addition to its presence in SF $[168,227]$. PRG4 has a multifaceted role in the joint and one aspect of its role is to provide boundary lubrication, to prevent friction-induced mitochondrial dysregulation and chondrocyte apoptosis [228-230]. Synthesis of PRG4 by cartilage and synovium was reduced in posttraumatic OA (PTOA) animal models and inflammatory cytokines $e . g$. IL-1 $\beta$ reduced PRG4 secretion by synovial fibroblasts while TGF- $\beta$ produced an opposite effect $[184,231,232]$. Furthermore, SF PRG4 levels decreased following acute joint trauma and in patients with advanced OA and rheumatoid arthritis (RA) [233-235]. Therapeutically, native and rhPRG4 demonstrated disease-modifying activities in pre-clinical PTOA mediated by their ability to reduce cartilage degeneration, enhance cartilage repair and reduce chondrocyte apoptosis [179, 236-238].

In this investigation, we studied the role of CD44 in mediating rhPRG4's antifibrotic and antimigratory biological effects on fibroblasts in response to exogenously introduced recombinant TGF- $\beta$ or TGF- $\beta$ produced by co-cultured macrophages in response to TLR 4 receptor stimulation. We identified that PRG4 and HA provide a similar outcome in TGF- $\beta$ stimulated OA FLS in the form of preventing transition of fibroblasts to myofibroblasts with inhibition of $\alpha$-SMA expression and thus stress fiber formation. PRG4 and HA produced an antifibrotic effect in OA FLS using concentrations that are well below what is normally found in SF [233-235]. As HA is the prototypical CD44 ligand, we used HA as a positive control and our findings support that CD44 modulates fibroblast to myofibroblast transition in OA synoviocytes. PRG4 was rapidly internalized by OA FLS, with a prominent role for CD44 in this process. However, CD44 was not the only mechanism by which OA synoviocytes internalized rhPRG4 as approximately $60 \%$ of rhPRG4 entry was blocked subsequent to CD44 receptor neutralization. CD44-mediated rhPRG4's uptake partially contributed to the latter's antifibrotic role as rhPRG4's effect on $\alpha$-SMA 
expression was significantly weakened, but not completely abolished, when CD44 mediated uptake was blocked. This indicates that CD44 engagement by rhPRG4 is biologically significant in the context of rhPRG4's antifibrotic effect but does not completely account for the manner in which rhPRG4 interacts with synoviocytes. CD44 also played a role in facilitating the enhanced migration of murine synovial fibroblasts that lack Prg4 expression as antibody-mediated neutralization of the receptor reduced cell migration. This finding is consistent with our prior observation that Prg4 null synoviocytes had higher CD44 receptor expression levels compared to Prg4 competent synoviocytes and is supported by an independent finding that CD44 plays a role in facilitating cell migration $[127,154,236]$. Synovial fibrosis developed in Prg4 null mice and expression of SMA, PLOD2 and collagen type-I progressively increased as animals aged. Interestingly, the in vivo antifibrotic effect due to Prg4 re-expression was not dependent on CD44 as endogenously produced PRG4 reduced SMA and PLOD2 expression in Cd44 null animals.

CD44 is a single-pass transmembrane glycoprotein that is widely expressed in immune and connective tissues, and different isoforms, generated by alternate splicing, have been characterized $[127,236]$. The extracellular domain of CD44 binds different ligands and thus can sense a broad array of microenvironmental signals resulting in ligand-specific effects in inflammation, cell migration and growth [239-241]. In addition to HA and PRG4, other CD44 ligands include osteopontin, chondroitin, fibronectin, and matrix metalloproteinases (MMPs) [239, 242]. The intracellular domain of CD44 lacks intrinsic kinase activity, but it transduces extracellular ligandspecific signals via a combination of proteolytic fragmentation or interactions with different signaling pathways including members of Src and Ras family of GTPases and protein phosphatase2A [239, 243, 244]. HA is cellularly uptaken by CD44, and this interaction mediates HA's ability to suppress MMP-13 and aggrecanase-1 expression in OA chondrocytes and synoviocytes [128, 
$245,246]$. In addition to inhibiting synovitis, HA treatment inhibited synovial fibrosis in a murine model of TGF- $\beta$ and treadmill running $[128,247,248]$. The effect of HA was CD44-dependent as its antifibrotic effect was abolished in CD44-deficient mice [247]. Contrary to HA, PRG4 had an in vivo antifibrotic effect that was not dependent on CD44. This might be related to PRG4's ability to regulate other signaling pathways apart from CD44. PRG4 binds to and inhibits the activation of TLR2 and TLR4 receptors by their ligands and by SF aspirates from patients with OA [249, 250]. In addition, PRG4 was internalized by murine macrophages and co-localized with both CD44 and to lesser extent TLR2 receptors in the cytosol of these cells [251]. As such, it is expected that the enhanced TLR2 and TLR4 receptor signaling in the absence of PRG4 would propagate synovitis in Prg4 null mice. The innate immune-mediated synovitis would in turn contribute to the development of synovial fibrosis. Upon Prg4 re-expression in 3-weeks old animals, PRG4 would attenuate TLR2 and TLR4 mediated synovitis, thereby potentially reducing TLR-regulated fibrotic tissue remodeling [252].

Myofibroblasts are characterized by $\alpha$-SMA expression, stress fiber and FAs formation and are thought to play an effector role in the pathophysiology of tissue fibrosis $[252,253]$. Compared to fibroblasts, myofibroblasts produce more collagen; respond to mitogenic signals resulting in cell proliferation, migration and secretion of matrix-degrading enzymes [190, 252]. While stress fibers and FAs are unique structures within the cell, they are highly interconnected, where they coordinate cell responses to mechanical signals in their microenvironment [254]. Interestingly, the mean size of FAs, and not their molecular composition or density, can accurately predict cell migration [255]. Normal synovial fibroblasts do not appear to express $\alpha-S M A$, even with TGF- $\beta$ stimulation [191]. In our study, OA FLS had a basal level of $\alpha$-SMA expression and in response to TGF- $\beta, \alpha$-SMA expression was enhanced and stress fibers appeared. This was also evident in 
murine NIH3T3 fibroblasts as the transition from a fibroblast to a myofibroblast phenotype was accompanied by stress fiber and FAs formation, with subcellular and cellular changes in the form of increased number and mean size of FAs and cell spread area and thus enhanced cell migration. rhPRG4 acted biologically to reduce the formation of stress fibers and to reduce the mean number and size of FAs in NIH3T3 fibroblasts and this reduction resulted in inhibition of fibroblast migration. Thus, it is unlikely that the observed antimigratory effect of rhPRG4 was a physical effect consequent to PRG4's amphipathic nature and its adsorption on hydrophilic and hydrophobic surfaces [198]. The higher mean size of FAs in synoviocytes from Prg4 null mice, and its resultant enhanced migration argues that PRG4 plays a role in regulating the expression of the cellular machinery mediating migration in vivo. This may have a biological relevance in the realm of synovial fibrosis where PRG4 may function to reduce the migration of fibrocytes to the inflamed joint and therefore the likelihood of adding to the pool of existing myofibroblasts in the synovium $[256,257]$.

Synovial tissue macrophages are critical regulators of the initiation, maintenance and resolution phases of synovitis [258]. The complex functions of macrophages are enabled by the presence of different macrophage subpopulations within the joint, which fulfill distinct inflammatory and antiinflammatory roles [258]. In synovial tissue fibrosis, bone marrow derived monocytes and macrophages are found in increased numbers, which may signal their involvement in the development of synovial fibrosis $[259,260]$. In response to an inflammatory signal, macrophages produce TGF- $\beta$ as a regulatory feedback signal to aid in the resolution of inflammation $[261,262]$. TGF- $\beta$ in turn triggers myofibroblast differentiation and production of matrix proteins [220]. In our study, LPS stimulation resulted in increased secretion of TGF- $\beta$ by macrophages. Cells secrete a latent form of TGF- $\beta$, which in turn is proteolytically converted to active TGF- $\beta$ by a variety of 
proteases, including MMPs [263]. The generation of active TGF- $\beta$ in the co-culture system was associated with fibroblast migration. Interestingly, the introduction of rhPRG4 after macrophage activation did not alter total TGF- $\beta$ quantity produced by macrophages. Furthermore, rhPRG4 did not alter the rate of activation of latent TGF- $\beta$, indicating that the antimigratory effect of rhPRG4 was at the level of fibroblasts' response to TGF- $\beta$ receptor activation. This is supported by our observation that rhPRG4 reduced Smad3 phosphorylation in TGF- $\beta$ reporter cells and that reduction in pSmad 3 intracellular levels attenuated the TGF- $\beta /$ Smad pathway activation. In TGF$\beta$ reporter cells, the attenuation of Smad signaling pathway was downstream to rhPRG4 uptake by these cells in a mechanism that involved CD44 receptor, similar to what was observed in OA FLS. However, the precise mechanism that is activated by CD44 engagement that inhibits TGF- $\beta / \mathrm{Smad}$ signaling pathway remains unclear. Another limitation of our study was that we did not study the antifibrotic effect of rhPRG4 in vivo.

\subsection{CONCLUSION}

In summary, we demonstrated that PRG4 is an important regulator of synovial tissue fibrosis using a combination of in vitro and in vivo models. PRG4 prevented the transition of human and murine fibroblasts to a myofibroblast phenotype and in OA FLS; the mechanism of PRG4's antifibrotic effect was partially linked to CD44-mediated cell uptake. rhPRG4 acted biologically to reduce Smad3 phosphorylation, the activation of TGF- $\beta /$ Smad signaling pathway, and FA mean size which translated to inhibiting fibroblast migration. The lack of Prg4 expression in murine synovial tissues resulted in enhanced expression of SMA, collagen type-I and PLOD2, the enzyme responsible for collagen cross-linking. Synovial fibrosis in $\operatorname{Prg} 4^{G T / G T}$ animals progressed with age and re-establishing Prg4 expression was antifibrotic. The role of PRG4 in regulating synovial fibrosis in vivo extends beyond its interaction with CD44 receptor, as Prg4 re-expression was 
antifibrotic in $C d 44$ null mice. Therapeutically, overexpressing PRG4 in the articular joint, using a viral mediated gene delivery approach, may prove beneficial in reducing synovial fibrosis in addition to reducing cartilage degeneration $[264,265]$. The study of PRG4 as an antifibrotic modulator of the joint's soft tissues is further warranted especially with the known contribution of synovial fibrosis in advanced OA.

\subsection{DECLARATIONS}

\subsection{Ethical Approval and Consent to Participate}

-OA synoviocytes were obtained from a commercial source (Cell Applications Inc.). Harvest of synoviocytes was performed following appropriate IRB approvals from partner sites with informed written consent from the donor. Patients were de-identified. Authors did not seek patients' consent to study these biological samples. This study was approved by the IRB at Chapman University, Orange, CA, USA.

-Harvest of synovial tissues from mice, and histological evaluations of mice joints were approved by the IACUC Committee at Rhode Island Hospital, Providence, RI, USA. All experiments were performed in accordance with established guidelines and regulations.

\subsection{Consent for Publication}

Not applicable

\subsection{Availability of Supporting Data}

Not applicable 


\subsection{Competing Interests}

Authors MQ, HR and LZ have nothing to disclose.

Author GJ authored patents on rhPRG4 and holds equity in Lubris LLC, MA, USA.

Author TS authored patents on rhPRG4, is a paid consultant for Lubris LLC, MA, USA and holds equity in Lubris LLC, MA, USA.

Author KE authored patents on rhPRG4.

All authors have no non-financial competing interests related to this manuscript.

\subsection{Funding}

This work is supported by R01AR067748 to KE and GJ.

\subsection{Authors' Contributions}

Authors MQ, GJ, LZ, and KE carried out experiments and participated in analysis of data. Authors TS and HR participated in study design and critical interpretation of results. Authors GJ and KE conceived the study and participated in data analysis and interpretation. All authors have participated in drafting and critical evaluation of the manuscript. All authors have read and approved the final version of the manuscript.

\subsection{Acknowledgements}

Not applicable 


\subsection{Figures}

A

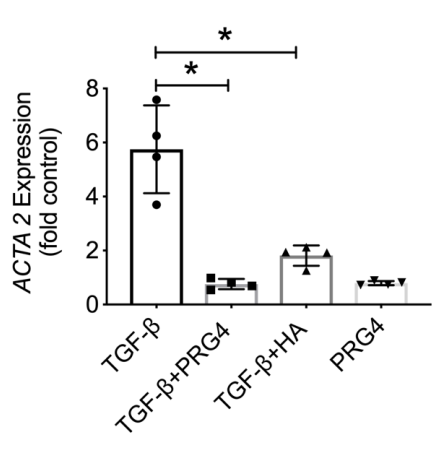

B

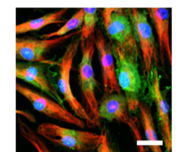

Control

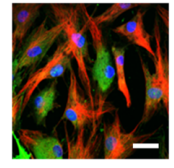

TGF- $\beta+$ PRG4
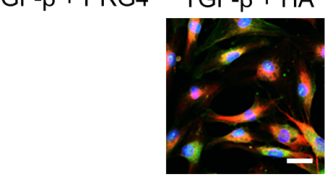

E

D
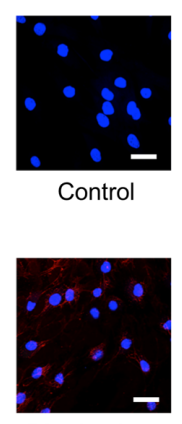

RhodaminerhPRG4 + Anti-CD44
PRG4

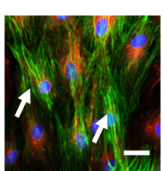

C

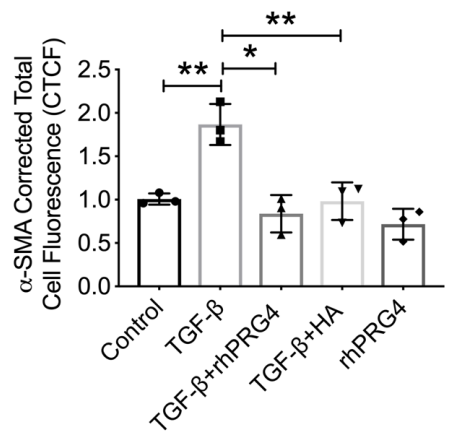

$\mathbf{F}$

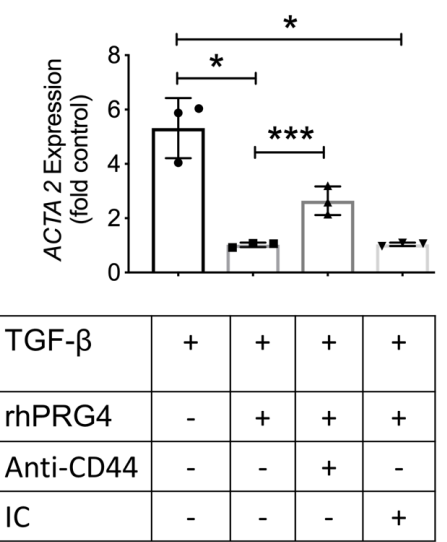

Fig. 4.1 Impact of proteoglycan-4 (PRG4) and high molecular weight hyaluronic acid (HA) treatments on TGF- $\beta 1$ induced alpha smooth muscle actin ( $\alpha$-SMA) expression (ACTA2), production and stress fiber formation in fibroblast-like synoviocytes isolated from patients with OA (OA FLS) undergoing knee replacement surgery and CD44-dependent uptake of rhodaminelabeled recombinant human PRG4 (rhPRG4) into OA FLS and associated regulation of ACTA2 expression. OA FLS were treated with TGF- $\beta 1(1 \mathrm{ng} / \mathrm{mL}) \pm \operatorname{rhPRG} 4(\sim 240 \mathrm{kDa})$ or HA $(\sim 1,200$ $\mathrm{kDa})(100 \mu \mathrm{g} / \mathrm{mL}$ for both treatments) for 24 hours followed by RNA isolation and qPCR using $G A P D H$ as an internal reference gene. OA FLS were stained with anti- $\alpha-S M A$ [198] and counterstained for $\alpha$-tubulin (red) and DAPI (blue). Corrected total cell fluorescence (CTCF) of $\alpha$-SMA was determined following a 48-hour treatment, and normalized to controls. Rhodaminelabeled rhPRG4 was incubated with OA FLS \pm anti-CD44 or isotype control (IC) antibodies for 
30 min., and CTFC was quantified. OA FLS were treated with TGF- $\beta 1 \pm$ rhPRG4 \pm anti-CD44 or IC for 24 hours and $A C T A 2$ expression was determined. Data are presented as a scatterplot with means and standard deviations highlighted utilizing OA FLS from 3-4 different patients. ${ }^{*} p<0.001 ; * * * p<0.05$. Scale $=50 \mu \mathrm{m}$.

A) PRG4 and HA reduced ACTA2 expression in OA FLS.

B) Representative images showing TGF- $\beta 1$ induced stress fiber formation in OA FLS (white arrows) and PRG4 or HA treatments prevented their formation.

C) PRG4 and HA reduced $\alpha$-SMA CTCF in OA FLS.

D) Representative images showing rhodemine-rhPRG4 intracellular localization (white arrows), following incubation with OA FLS.

E) Co-incubation of rhPRG4 and anti-CD44 reduced cellular uptake of rhPRG4.

F) Anti-CD44 antibody co-treatment reduced rhPRG4's antifibrotic effect in OA FLS. 


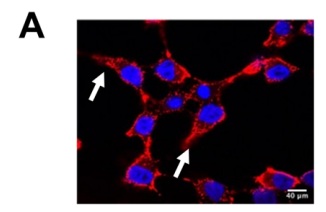

в

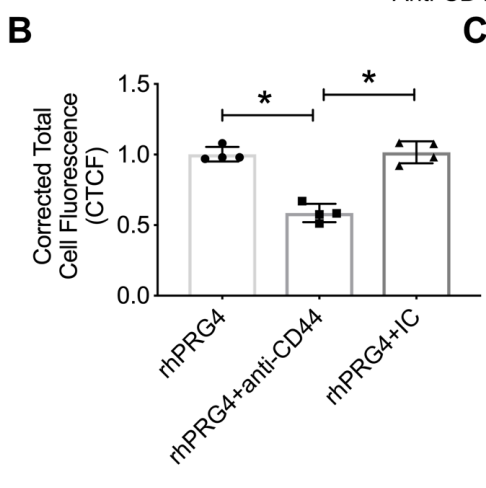

D

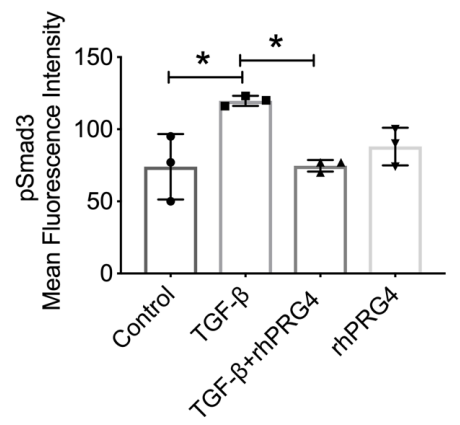

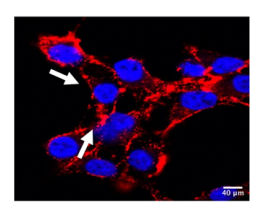

RhodaminerhPRG4 + IC

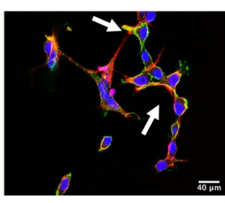

Merged Image

Red: Rhodamine-rhPRG4 Green: CD44

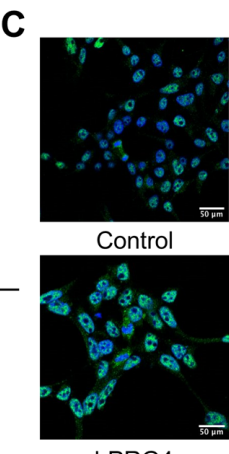

rhPRG4

E

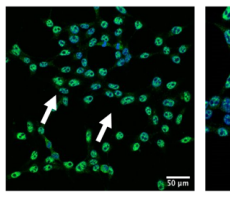

TGF- $\beta$

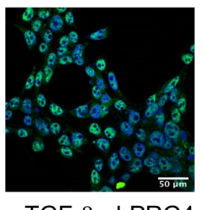

TGF- $\beta+$ rhPRG4
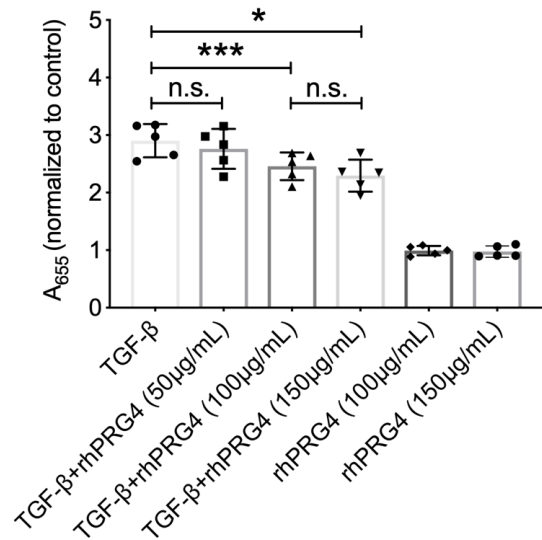

Fig. 4.2 CD44-dependent interaction of recombinant human proteoglycan-4 (rhPRG4) with HEK Blue-TGF- $\beta$ cells and associated modulation of phosphorylated Smad3 (pSmad3) and TGF$\beta /$ Smad signaling pathway. HEK Blue-TGF- $\beta$ is an engineered cell line produced by transfecting human embryonic kidney (HEK) cells with TGF- $\beta$ receptor 1 (TGF- $\beta$ R1), Smad3 and Smad4 genes. Activation of TGF- $\beta$ R1 results in expression of secreted alkaline phosphatase (SEAP) whose activity can be determined colorimetrically. Rhodamine-labeled rhPRG4 was incubated with HEK Blue-TGF- $\beta$ cells \pm anti-CD44 or isotype control (IC) antibodies and corrected total cell fluorescence (CTCF) was determined. pSmad3 immunocytostaining was performed using an antibody against pSmad3 and pSmad3 fluorescence intensity was determined. Activity of TGF$\beta /$ Smad pathway was determined colorimetrically. ${ }^{*} p<0.001 ; *^{*}<<0.01 ;$ n.s.: non-significant. Scale in $A=40 \mu \mathrm{m}$; Scale in $\mathrm{C}=50 \mu \mathrm{m}$.

A) Representative images showing rhPRG4 internalization (white arrows) by HEK-TGF- $\beta$ cells and colocalization with CD44 receptor (white arrows in merged image).

B) rhPRG4 uptake by HEK-TGF- $\beta$ cells was reduced by CD44 receptor neutralization.

C) Representative images showing pSmad3 staining in HEK-TGF- $\beta$ cells following TGF- $\beta$ stimulation (white arrows) \pm rhPRG4 treatment.

D) pSmad3 immunocytostaining was reduced following rhPRG4 treatment.

E) rhPRG4 treatment dose-dependently reduced TGF- $\beta /$ Smad signaling pathway in TGF- $\beta$ stimulated HEK- TGF- $\beta$ cells. 
A

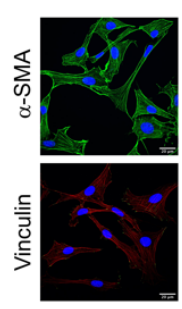

Control
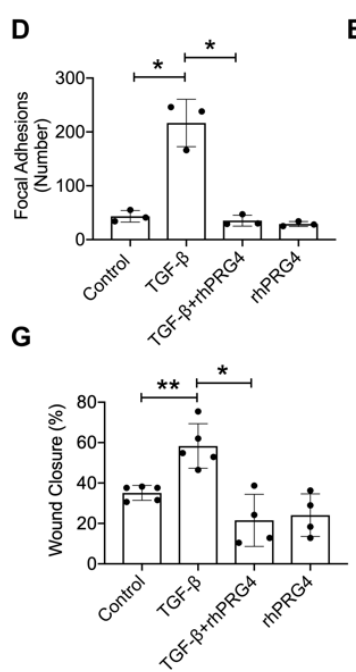

B

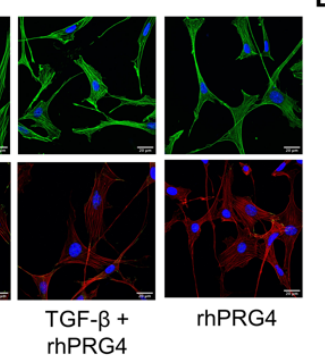

E

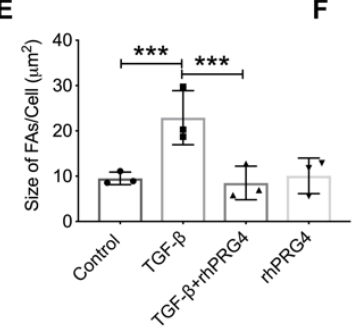

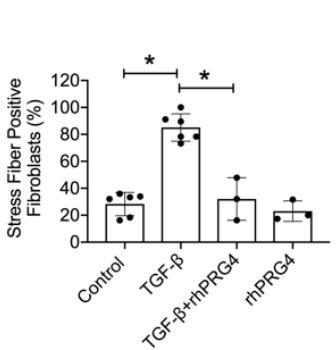

C
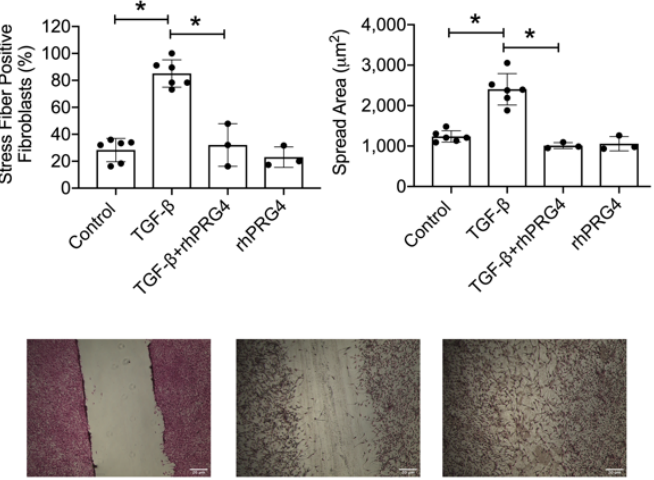

Baseline

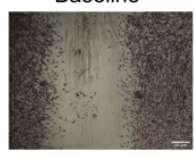

TGF- $\beta+$ rhPRG 4

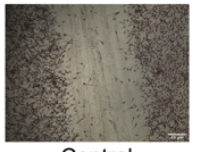

Control

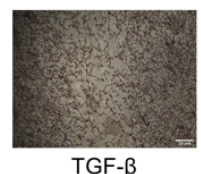

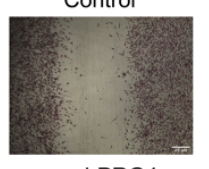

rhPRG4

Fig. 4.3 Impact of recombinant human proteoglycan-4 (rhPRG4) treatment on TGF- $\beta$ induced stress fiber formation, vinculin expression, and formation of focal adhesions (FAs) in murine fibroblasts (NIH3T3) and its relationship to cell migration. Stress fiber formation was probed using an anti-alpha smooth muscle actin ( $\alpha$-SMA) antibody and a blinded investigator evaluated $\%$ stress fiber-positive fibroblasts. Vinculin (a marker of FAs) expression was probed using an anti-vinculin antibody and cells were counterstained using rhodamine-labeled phalloidin (cytoskeleton label; red). NIH3T3 Fibroblast migration was performed using a scratch assay and the wound closure percentage was determined. ${ }^{*} p<0.001 ; * *^{*}<0.01 ; * * * p<0.05 ;$ n.s.: nonsignificant. Scale in $\mathrm{A}$ and $\mathrm{F}=20 \mu \mathrm{m}$;

A) Representative images showing TGF- $\beta$ induced stress fiber formation and vinculin staining (white arrows) in NIH3T3 fibroblasts.

B) rhPRG4 reduced stress fiber formation in NIH3T3 fibroblasts.

C) rhPRG4 reduced mean NIH3T3 fibroblast spread area.

D) rhPRG4 reduced the number of FAs in NIH3T3 fibroblasts.

E) rhPRG4 reduced the mean size of FAs in NIH3T3 fibroblasts.

F) Representative images showing NIH3T3 migration in response to TGF- $\beta \pm$ rhPRG4.

G) rhPRG4 reduced NIH3T3 migration. 

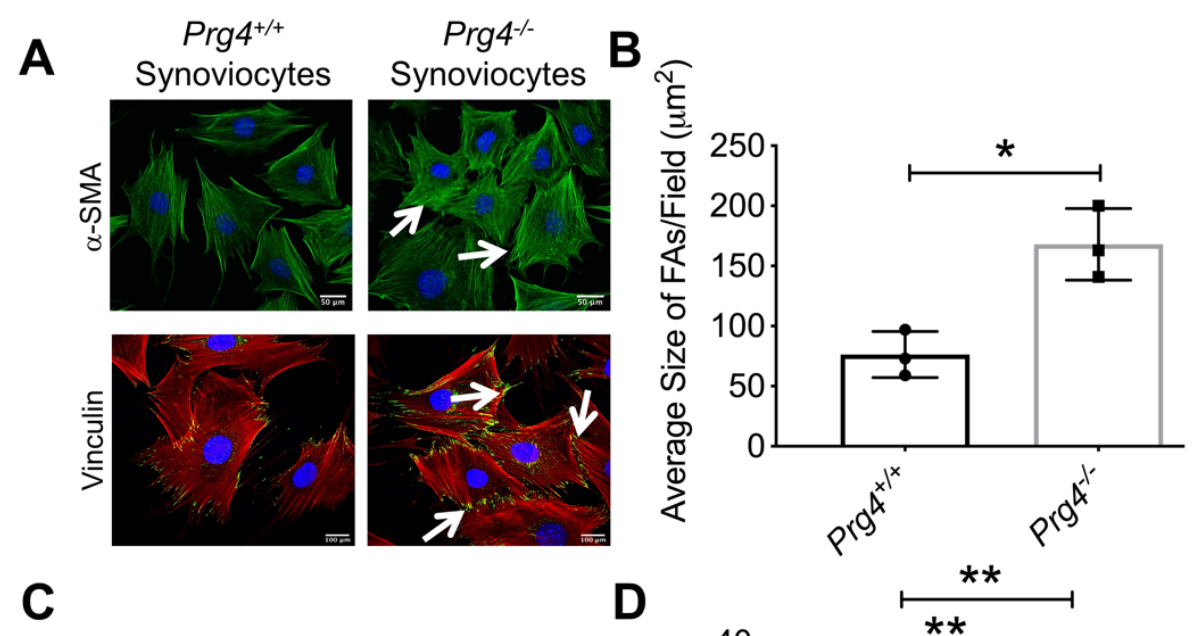

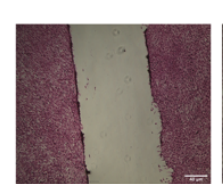

Baseline

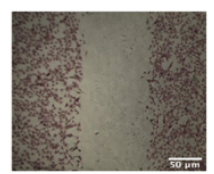

rhPRG4

$\left(\operatorname{Prg} 4^{-/}\right)$

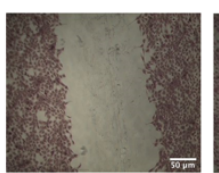

$\mathrm{Prg}^{+/+}$ Synoviocytes

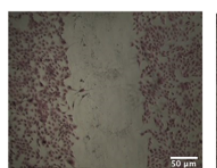

Anti-CD44

$\left(\right.$ Prg $\left.^{-/}\right)$

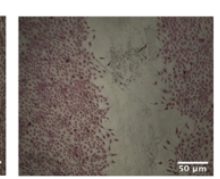

Prg4\%Synoviocytes

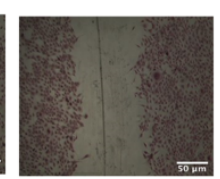

IC

(Prg4 $\left.{ }^{-/}\right)$
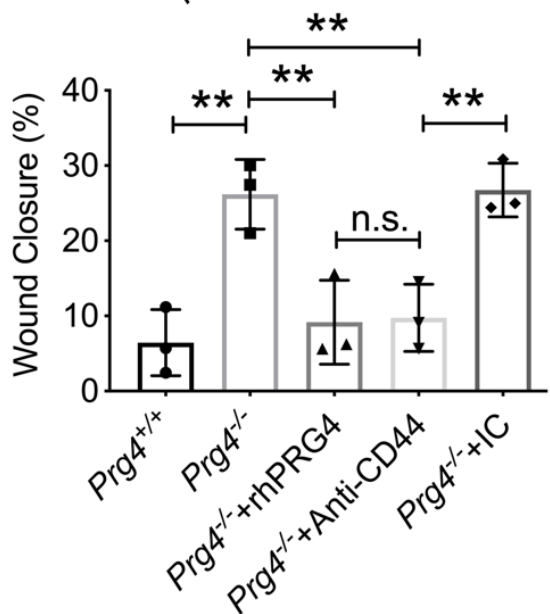

Fig. 4.4 Stress fibers and focal adhesions (FAs) in murine synovial fibroblasts isolated from Prg4${ }^{-}$and $\operatorname{Prg}^{+/+}$mice and their relationship to cell migration. Stress fiber formation was probed using an anti-alpha smooth muscle actin ( $\alpha$-SMA) antibody [198]. Vinculin (a marker of FAs) expression was probed using an anti-vinculin antibody [198] and cells were counterstained using rhodamine-labeled phalloidin (cytoskeleton label; red). The impact of rhPRG4 treatment $(200 \mu \mathrm{g} / \mathrm{mL})$ on basal $\operatorname{Prg} 4^{-/}$synovial fibroblast migration was assessed using the scratch assay and the wound closure percentage was determined. To highlight the CD44-dependency of the enhanced basal migration of $\mathrm{Prg}^{-/}$synovial fibroblasts, cells were also treated with either an antiCD44 or isotype control (IC) antibodies ( $2 \mu \mathrm{g} / \mathrm{mL}$ for both antibodies). ${ }^{*} p<0.001 ;{ }^{* *} p<0.01 ; n$.s.: non-significant. Scale in $\mathrm{A}=50 \mu \mathrm{m}(\alpha-\mathrm{SMA})$ and $100 \mu \mathrm{m}$ (vinculin); Scale in $\mathrm{C}=50 \mu \mathrm{m}$.

A) Representative images showing increased stress fibers and FAs in $\mathrm{Prg}^{-/-}$synoviocytes.

B) $\operatorname{Prg}^{-/}$synoviocytes demonstrated a higher mean FA size than $\operatorname{Prg}^{+/+}$synoviocytes.

C) Representative images showing enhanced basal migration of $\mathrm{Prg}^{-/-}$synoviocytes compared to $\operatorname{Prg} 4^{+/+}$synoviocytes.

D) rhPRG4 and anti-CD44 treatments were equally effective in reducing basal migration of $\operatorname{Prg}^{-/}$synoviocytes. 


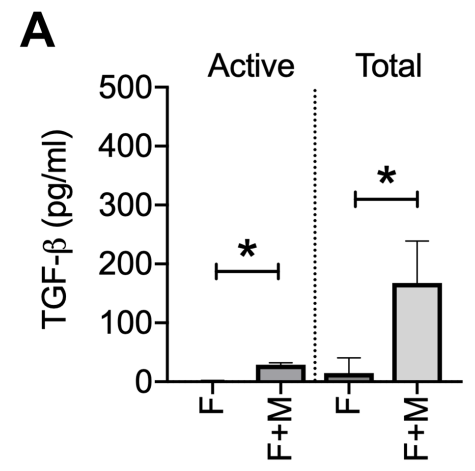

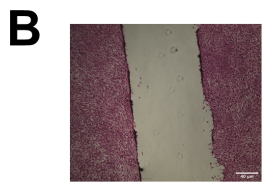

Baseline

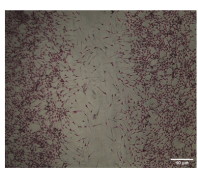

$\mathrm{F}+\mathrm{M}+$ rhPRG4

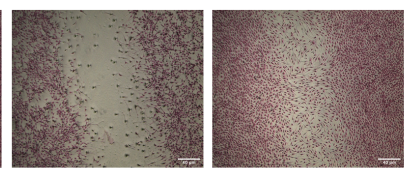

$\mathrm{F}$

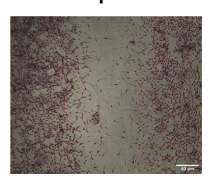

rhPRG4
$F+M$

Active

Total
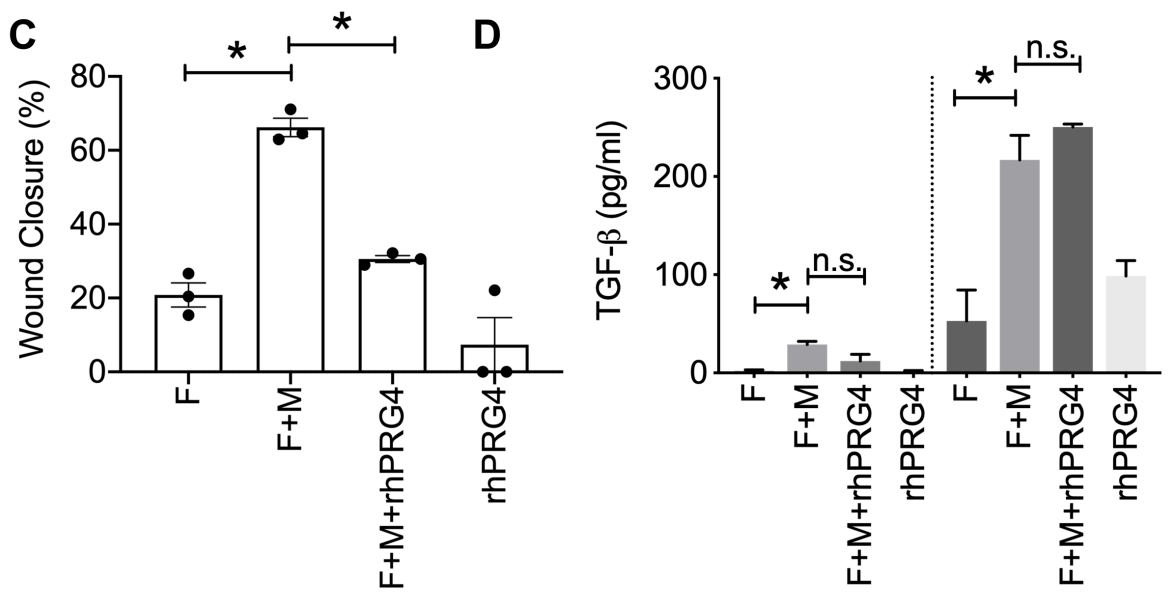

Fig. 4.5 Impact of co-culturing murine fibroblasts $(\mathrm{F})$ and murine macrophages $(\mathrm{M})$ on active and total supernatant TGF- $\beta$ levels, fibroblast migration and efficacy of rhPRG4 in regulating fibroblast migration in the co-culture model. Murine macrophages (J774A) were stimulated with lipopolysaccharide (LPS; $5 \mu \mathrm{g} / \mathrm{mL}$ ) for 24 hours prior to seeding in the top chamber of a co-culture system. Murine fibroblasts (NIH3T3) were cultured in the lower chamber of the same system. Active and total TGF- $\beta$ media levels were determined using an ELISA. A scratch was performed in the fibroblast monolayer and fibroblast migration in the lower chamber was determined at 48 hours \pm rhPRG4 $(200 \mu \mathrm{g} / \mathrm{mL}) .{ }^{*} p<0.001$; $* * p<0.01$; n.s.: nonsignificant. Scale $=40 \mu \mathrm{m}$.

A) Active and total TGF- $\beta$ concentrations were higher in the fibroblast and macrophage co-culture compared to fibroblasts alone.

B) Representative images of fibroblast migration across different experimental groups.

Fibroblast migration was highest in co-cultured fibroblasts and macrophages and rhPRG4 treatment reduced fibroblast migration.

C) rhPRG4 reduced fibroblast migration in a fibroblast and macrophage co-culture model.

D) rhPRG4 did not alter active or total TGF- $\beta$ media levels in the fibroblast and macrophage co-culture model. 

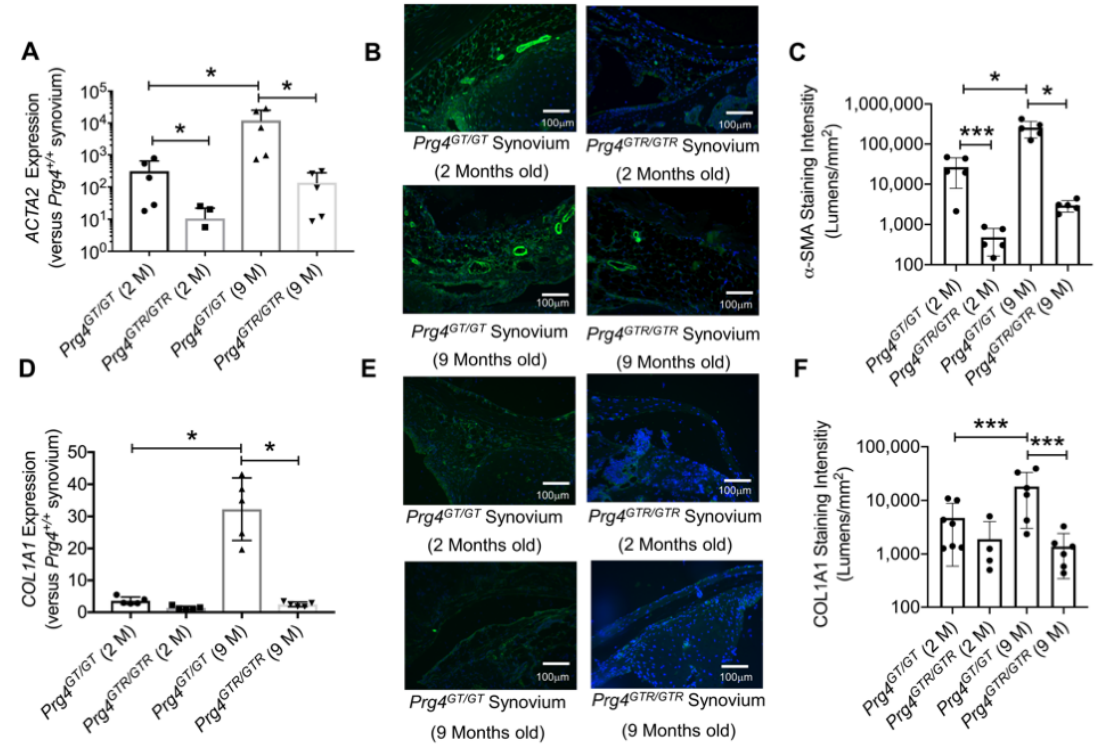

E
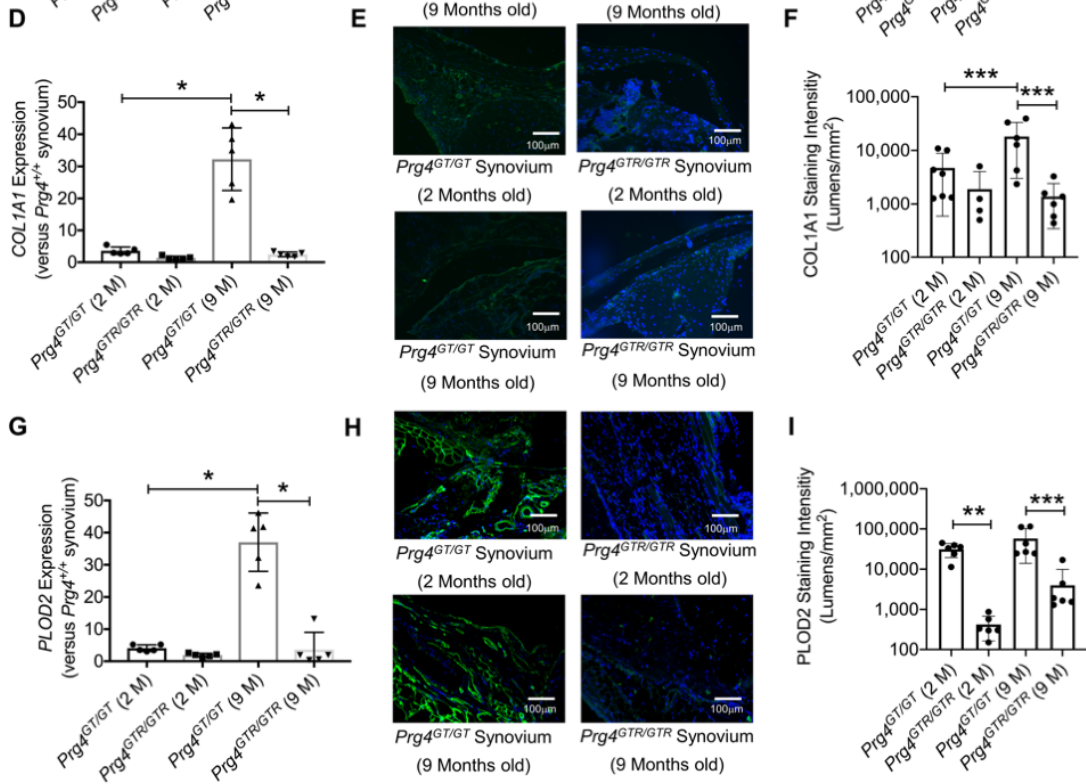

$\mathbf{H}$
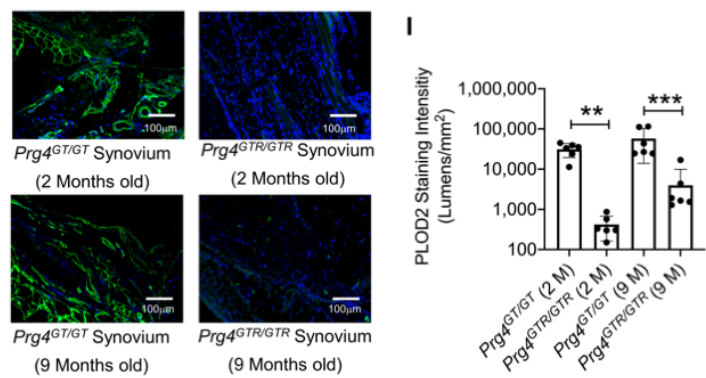

Fig. 4.6 Gene expression and immunohistological analysis of fibrotic markers: alpha smooth muscle actin ( $\alpha$-SMA) (ACTA2), collagen type-1 (COL1A1) and procollagen-lysine, 2oxoglutarate 5-dioxygenase (PLOD2) in synovial tissues isolated from 2- and 9-months old gene$\operatorname{trap}\left(\operatorname{Prg} 4^{G T / G T}\right)$ and 2 and 9 months old recombined gene-trap $\left(\operatorname{Prg} 4^{G T R / G T R}\right)$ mice. The $P r g 4$ genetrap allele is a loss of function allele $\left(\operatorname{Prg} 4^{G T / G T}\right)$ whose function is restored with $\mathrm{Cre}$-excision $\left(\right.$ Prg4 $\left.{ }^{G T R / G T R}\right)$. Recombination occurred at 3 weeks of age using intraperitoneal tamoxifen $(0.1$ $\mathrm{mg} / \mathrm{g}$ in $100 \mu \mathrm{L}$ corn oil vehicle) daily for 10 days. Fibrotic markers were probed using specific antibodies and staining intensities (lumens per $\mathrm{mm}^{2}$ ) were quantified. $* p<0.001 ; * * p<0.01$; $* * * p<0.05$. Scale $=100 \mu \mathrm{m}$.

A) ACTA2 expression was higher in 9 months $\operatorname{Prg} 4^{G T / G T}$ compared to 2 months littermates and was reduced by $\operatorname{Prg} 4$ re-expression.

B) Representative images showing enhanced $\alpha$-SMA immunostaining in 2 and 9 months $P r g 4^{G T / G T}$ synovia.

C) Prg4 re-expression reduced $\alpha$-SMA content in 2 and 9-months old animals.

D) COL1A1 expression was higher in 9 months old $\operatorname{Prg} 4^{G T / G T}$ animals compared to 2 months and was reduced by $\mathrm{Prg} 4$ re-expression. 
E) Representative images showing enhanced collagen type I immunostaining in 9 months old $\operatorname{Prg} 4^{G T / G T}$ synovia.

F) Collagen type I content was higher in 9 months old $\operatorname{Prg} 4^{G T / G T}$ animals and was reduced with Prg4 re-expression.

G) PLOD2 expression was higher in 9 months old $\operatorname{Prg} 4^{G T / G T}$ animals compared to 2 months and was reduced by $\operatorname{Prg} 4$ re-expression.

H) Representative images showing enhanced PLOD2 immunostaining in $\operatorname{Prg} 4^{G T / G T}$ synovia.

I) Prg4 re-expression reduced PLOD2 content in $\operatorname{Prg} 4{ }^{G T / G T}$ synovia in 2- and 9-months old animals. 


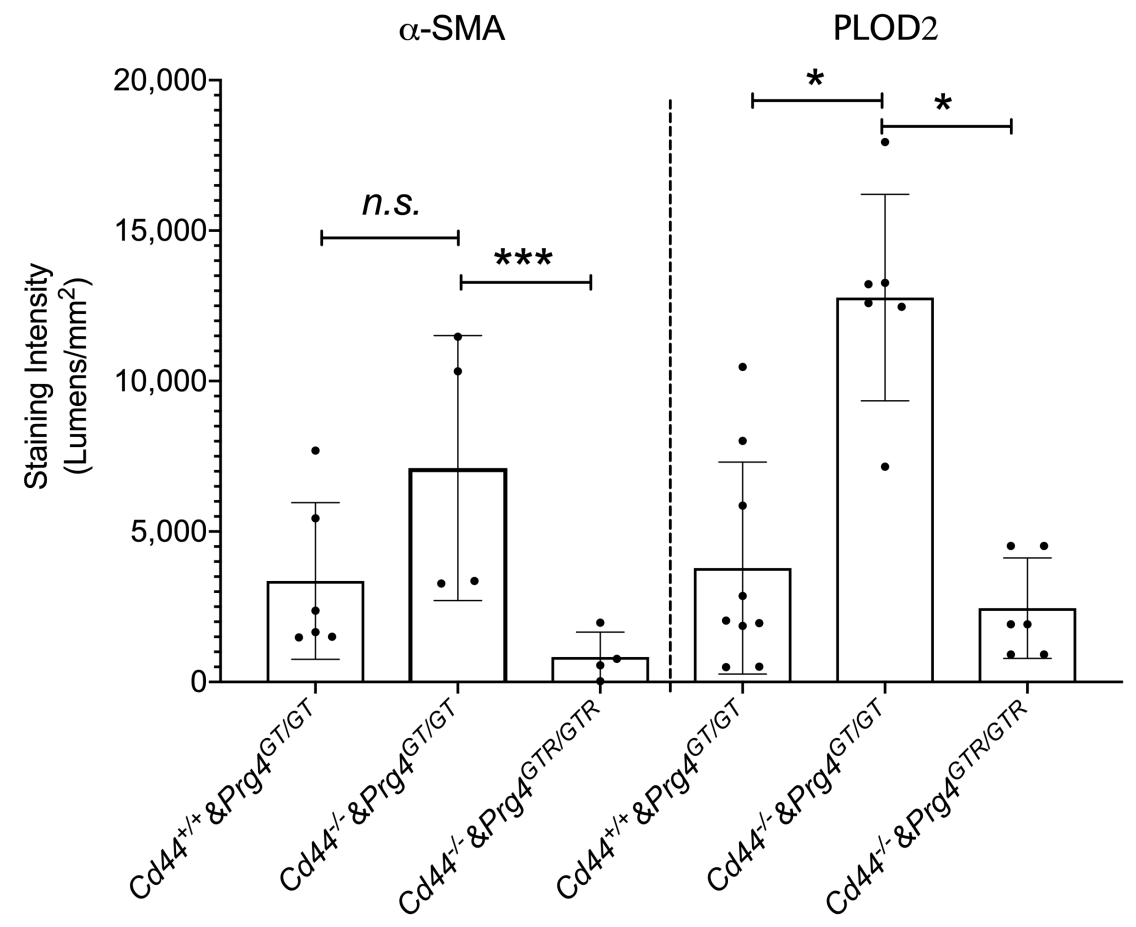

Fig. 4.7 Modulation of expression of fibrotic markers: alpha smooth muscle actin ( $\alpha$-SMA) and procollagen-lysine, 2-oxoglutarate 5-dioxygenase (PLOD2) in synovial tissues from Prg4 genetrap animals by CD44. The Prg4 gene-trap allele is a loss of function allele (Prg4 $\left.{ }^{G T / G T}\right)$ whose function is restored with $C r e$-excision $\left(\operatorname{Prg} 4^{G T R / G T R}\right)$. Prg4 ${ }^{G T / G T}$ animals were crossed with $C d 44^{-}$ /- mice to generate $\operatorname{Prg}^{G T / G T} \& C d 44^{-/}$animals. Recombination occurred at 3 weeks of age using intraperitoneal tamoxifen $(0.1 \mathrm{mg} / \mathrm{g}$ in $100 \mu \mathrm{L}$ corn oil vehicle) daily for 10 days. Fibrotic markers were probed using specific antibodies and staining intensities (lumens per $\mathrm{mm}^{2}$ ) were quantified. Prg4 recombination reduced $\alpha$-SMA and PLOD2 staining in synovial tissues of 2-months old $C d 44^{+/+}$and $C d 44^{-/}$animals. ${ }^{*} p<0.001 ; * * * p<0.05$; n.s.: non-significant. 


\section{CHAPTER 5}

\section{SUMMARY AND CONCLUSION}

In chapter 2, given the importance of CD44 in OA pathogenesis, and the emerging evidence of a biological role for HA, we aimed to evaluate the role of CD44 in regulating NF- $\mathrm{KB}$ activation and proinflammatory cytokine production in response to TLR2 receptor activation in human macrophages, using a combination of CD44 receptor knockdown, TNF- $\alpha$ production by macrophages from CD44 wildtype and knockout mice, and CD44 receptor engagement by CD44neutralizing $\mathrm{Ab}$ and $\mathrm{HA}$ treatments. HA is produced by fibroblast-like synoviocytes and is a major component of the synovial fluid (SF). HA exerts important functions in the joint and its biological effects are mediated by its binding to its transmembrane receptor, CD44. In this work, we found that Anti-CD44 Ab and HA treatments reduced NF- $\kappa B$ translocation, IL-1 $\beta$ and TNF- $\alpha$ expression, and production. Inhibition of proinflammatory response in macrophages by HA was mediated by CD44. Protein phosphatase 2A mediated the reduction in NF-kB translocation by HA. CD44 knockdown reduced NF- $\kappa B$ nuclear translocation and downstream IL- $1 \beta$ and TNF- $\alpha$ protein production following TLR2 receptor stimulation. $C d 44^{+/+}$murine bone marrow-derived macrophages produced higher TNF- $\alpha$ compared with $C d 44^{-/-}$macrophages following TLR2 stimulation. HA dose-dependently inhibited TLR2-induced TNF- $\alpha$ production by murine bone marrow-derived macrophages. OA SF stimulated TLR2 and TLR4 receptors and induced NF- $\mathrm{KB}$ translocation in human macrophages. Anti-CD44 Ab treatment significantly reduced macrophage activation by OA SF. CD44 regulated TLR2 responses in human macrophages, whereby a 
reduction in CD44 levels or engagement of CD44 by its ligand (HA) or a CD44-specific $\mathrm{Ab}$ reduced NF-kB translocation and downstream proinflammatory cytokine production. A CD44specific $\mathrm{Ab}$ reduced macrophage activation by $\mathrm{OA} \mathrm{SF}$, and $\mathrm{CD} 44$ is a potentially novel target in OA treatment.

In chapter 3, we investigated the antifibrotic efficacy of forskolin, an adenylyl cyclase activator that increases intracellular cAMP, in a model of TGF- $\beta$ stimulated osteoarthritic fibroblast-like synoviocytes (OA-FLS). Synovial fibrosis is characterized by excess extracellular collagen I, TIMP-1 and PLOD2 expression and FLS proliferation and migration. A myofibroblast-like differentiation of synoviocytes, characterized by alpha smooth muscle actin ( $\alpha$-SMA) expression, is associated with synovial fibrosis. Synoviocytes secrete HA and proteoglycan-4 (PRG4). HA and PRG4 may be linked to synovial fibrosis. Collagen I (COL1A1), $\alpha$-SMA, TIMP-1, PLOD2 expression and procollagen I, $\alpha$-SMA, HA and PRG4 production, migration and proliferation of OA-FLS were studied in TGF- $\beta \pm$ forskolin-treated OA-FLS. The antifibrotic effect of HA and PRG4 was investigated using OA-FLS and $\mathrm{Prg}^{-/}$synoviocytes, and fibrosis markers were evaluated in $\mathrm{Prg}^{+/+}$and $\mathrm{Prg}^{-/}$synovial tissues. Forskolin $(10 \mu \mathrm{M})$ reduced COL1A1, $\alpha$-SMA and TIMP-1 expression, with no change in PLOD2 expression. Forskolin $(10-30 \mu \mathrm{M})$ reduced procollagen I, $\alpha$-SMA and OA-FLS migration and proliferation. Forskolin $(10 \mu \mathrm{M})$ increased HA secretion, PRG4 expression and production. HA and synoviocyte PRG4 reduced $\alpha$-SMA expression and content. PRG4 reduced COL1A1 expression and procollagen I content. Prg4-/synovial tissues exhibited increased $\alpha$-SMA, COL1A1 and TIMP-1 expression compared to $\operatorname{Prg} 4^{+/+}$tissues. $\operatorname{Prg} 4^{-/}$synoviocytes demonstrated strong $\alpha$-SMA and collagen type I staining with none detected in $\mathrm{Prg}^{+/+}$synoviocytes and PRG4 treatment reduced cellular staining for both proteins. Clinical significance: OA synovial fibrosis can be mitigated by therapeutically increasing 
intracellular cAMP in synovial fibroblasts with an associated enhancement of HA and PRG4 production.

In chapter 4, we demonstrated that PRG4 is an important regulator of synovial tissue fibrosis using a combination of in vitro and in vivo models. PRG4 prevented the transition of human and murine fibroblasts to a myofibroblast phenotype and in OA FLS; the mechanism of PRG4's antifibrotic effect was partially linked to CD44- mediated cell uptake. rhPRG4 acted biologically to reduce Smad3 phosphorylation, the activation of TGF- $\beta$ / Smad signaling pathway, and FA mean size which translated to inhibiting fibroblast migration. rhPRG4 inhibited fibroblast migration in a macrophage and fibroblast co-culture model without altering active or total TGF- $\beta$ levels. The lack of Prg4 expression in murine synovial tissues resulted in enhanced expression of SMA, collagen type-I, and PLOD2, the enzyme responsible for collagen cross-linking. Synovial fibrosis in $P r g 4 G T / G T$ animals progressed with age and re-establishing Prg4 expression was antifibrotic. The role of PRG4 in regulating synovial fibrosis in vivo extends beyond its interaction with CD44 receptor, as Prg4 re-expression was antifibrotic in Cd44 null mice. Therapeutically, overexpressing PRG4 in the articular joint, using a viral mediated gene delivery approach, may prove beneficial in reducing synovial fibrosis in addition to reducing cartilage degeneration. The study of PRG4 as an antifibrotic modulator of the joint's soft tissues is further warranted especially with the known contribution of synovial fibrosis in advanced OA. 


\section{REFERENCES}

1. Scanzello, C.R. and S.R.J.B. Goldring, The role of synovitis in osteoarthritis pathogenesis. 2012. 51(2): p. 249-257.

2. Xia, B., et al., Osteoarthritis pathogenesis: a review of molecular mechanisms. 2014. 95(6): p. 495-505.

3. Shen, J. and D.J.T.J.o.t.A.A.o.O.S. Chen, Recent progress in osteoarthritis research. 2014. 22(7): p. 467.

4. Heidari, B.J.C.j.o.i.m., Knee osteoarthritis prevalence, risk factors, pathogenesis and features: Part I. 2011. 2(2): p. 205.

5. Martel-Pelletier, J.J.O. and cartilage, Pathophysiology of osteoarthritis. 1999. 7(4): p. 371373.

6. Goldring, S., M.J.J.o.M. Goldring, and N. Interactions, Clinical aspects, pathology and pathophysiology of osteoarthritis. 2006. 6(4): p. 376.

7. Iannone, F., G.J.A.c. Lapadula, and e. research, The pathophysiology of osteoarthritis. 2003. 15(5): p. 364-372.

8. $\quad$ Smith, M.D.J.T.o.r.j., Suppl 1: The Normal Synovium. 2011. 5: p. 100.

9. Sophia Fox, A.J., A. Bedi, and S.A.J.S.h. Rodeo, The basic science of articular cartilage: structure, composition, and function. 2009. 1(6): p. 461-468.

10. Kapoor, M., et al., Role of proinflammatory cytokines in the pathophysiology of osteoarthritis. 2011. 7(1): p. 33.

11. Mathiessen, A., P.G.J.A.r. Conaghan, and therapy, Synovitis in osteoarthritis: current understanding with therapeutic implications. 2017. 19(1): p. 18.

12. Sellam, J. and F.J.N.R.R. Berenbaum, The role of synovitis in pathophysiology and clinical symptoms of osteoarthritis. 2010. 6(11): p. 625.

13. Goldring, S.R., M.B.J.C.O. Goldring, and R. Research ${ }^{\circledR}$, The role of cytokines in cartilage matrix degeneration in osteoarthritis. 2004. 427: p. S27-S36.

14. Kataoka, Y., et al., Mechanisms involved in suppression of ADAMTS4 expression in synoviocytes by high molecular weight hyaluronic acid. 2013. 432(4): p. 580-585.

15. Inada, M., et al., Critical roles for collagenase-3 (Mmp13) in development of growth plate cartilage and in endochondral ossification. 2004. 101(49): p. 17192-17197.

16. Stickens, D., et al., Altered endochondral bone development in matrix metalloproteinase 13-deficient mice. 2004. 131(23): p. 5883-5895. 
17. Glasson, S.S., et al., Deletion of active ADAMTS5 prevents cartilage degradation in a murine model of osteoarthritis. 2005. 434(7033): p. 644-648.

18. Lewis, J., et al., Acute joint pathology and synovial inflammation is associated with increased intra-articular fracture severity in the mouse knee. 2011. 19(7): p. 864-873.

19. Loeuille, D., et al., Macroscopic and microscopic features of synovial membrane inflammation in the osteoarthritic knee: correlating magnetic resonance imaging findings with disease severity. 2005. 52(11): p. 3492-3501.

20. Sinusas, K.J.A.f.p., Osteoarthritis: diagnosis and treatment. 2012. 85(1): p. 49-56.

21. Cheng, C., J. Tian, and F.J.A.o.t.r.d. Zhang, Can IL-1 be used as a target for osteoarthritis? 2019: p. annrheumdis-2019-215513.

22. Lawrence, D.A.J.E.c.n., Transforming growth factor-beta: a general review. 1996. 7(3): p. 363-374.

23. Weiss, A. and L.J.W.I.R.D.B. Attisano, The TGFbeta superfamily signaling pathway. 2013. 2(1): p. 47-63.

24. Van der Kraan, P., et al., TGF-beta signaling in chondrocyte terminal differentiation and osteoarthritis: modulation and integration of signaling pathways through receptor-Smads. 2009. 17(12): p. 1539-1545.

25. Arntz, O.J.L.i., a.j.o.t. methods, and pathology, Transforming growth factor-beta 1 stimulates articular chondrocyte proteoglycan synthesis and induces osteophyte formation in the murine knee joint. 1994. 71(2): p. 279-290.

26. Davidson, E.B., et al., TGF- $\beta$ and osteoarthritis. 2007. 15(6): p. 597-604.

27. Cen, X., S. Liu, and K.J.F.i.p. Cheng, The role of toll-like receptor in inflammation and tumor immunity. 2018. 9: p. 878.

28. Chen, K., et al., Toll-like receptors in inflammation, infection and cancer. 2007. 7(10): p. 1271-1285.

29. Piccinini, A. and K.J.M.o.i. Midwood, DAMPening inflammation by modulating TLR signalling. 2010. 2010.

30. Huang, Q.-Q., R.M.J.A.o.b. Pope, and biophysics, The role of glycoprotein 96 in the persistent inflammation of rheumatoid arthritis. 2013. 530(1): p. 1-6.

31. Lees, S., et al., Bioactivity in an aggrecan 32-mer fragment is mediated via Toll-like receptor 2. 2015. 67(5): p. 1240-1249.

32. Okamura, Y., et al., The extra domain A of fibronectin activates Toll-like receptor 4. 2001. 276(13): p. 10229-10233. 
33. Schaefer, L., et al., The matrix component biglycan is proinflammatory and signals through Toll-like receptors 4 and 2 in macrophages. 2005. 115(8): p. 2223-2233.

34. Gómez, R., et al., TLR4 signalling in osteoarthritis-finding targets for candidate DMOADs. 2015. 11(3): p. 159.

35. Lasker, M.V. and S.K.J.T.J.o.I. Nair, Intracellular TLR signaling: a structural perspective on human disease. 2006. 177(1): p. 11-16.

36. O'Neill, L.A.J.I.r., The interleukin-1 receptor/Toll-like receptor superfamily: 10 years of progress. 2008. 226(1): p. 10-18.

37. Jung, Y.O., et al., Toll-like receptor 2 and 4 combination engagement upregulate IL-15 synergistically in human rheumatoid synovial fibroblasts. 2007. 109(1): p. 21-27.

38. Jung, Y.O., et al., Synergism of Toll-like receptor 2 (TLR2), TLR4, and TLR6 ligation on the production of tumor necrosis factor (TNF)- $\alpha$ in a spontaneous arthritis animal model of interleukin (IL)-1 receptor antagonist-deficient mice. 2009. 123(2): p. 138-143.

39. Nair, A., et al., Synovial fluid from patients with early osteoarthritis modulates fibroblastlike synoviocyte responses to Toll-like receptor 4 and Toll-like receptor 2 ligands via soluble CD14. 2012. 64(7): p. 2268-2277.

40. Alquraini, A., et al., The interaction of lubricin/proteoglycan 4 (PRG4) with toll-like receptors 2 and 4: an anti-inflammatory role of PRG4 in synovial fluid. 2015. 17(1): p. 353.

41. Stifano, G., et al., Chronic Toll-like receptor 4 stimulation in skin induces inflammation, macrophage activation, transforming growth factor beta signature gene expression, and fibrosis. 2014. 16(4): p. R136.

42. Krieg, T., et al., Fibrosis in connective tissue disease: the role of the myofibroblast and fibroblast-epithelial cell interactions. 2007. 9(2): p. S4.

43. Cheuy, V.A., et al., Arthrofibrosis associated with total knee arthroplasty. 2017. 32(8): p. 2604-2611.

44. Jeroen, C., et al., Early follow-up after primary total knee and total hip arthroplasty with rapid recovery: focus groups. 2015. 81: p. 447-453.

45. Van Der Kraan, P.M.J.N.R.R., The changing role of TGF $\beta$ in healthy, ageing and osteoarthritic joints. 2017. 13(3): p. 155.

46. Davidson, E.N.B., et al., TGF $\beta$-induced cartilage repair is maintained but fibrosis is blocked in the presence of Smad7. 2006. 8(3): p. R65. 
47. Remst, D., et al., Osteoarthritis-related fibrosis is associated with both elevated pyridinoline cross-link formation and lysyl hydroxylase $2 b$ expression. 2013. 21(1): p. 157164.

48. Remst, D., et al., Gene Expression Analysis of Murine and Human Osteoarthritis Synovium Reveals Elevation of Transforming Growth Factor $\beta$-Responsive Genes in OsteoarthritisRelated Fibrosis. 2014. 66(3): p. 647-656.

49. Wu, C.L., et al., Conditional macrophage depletion increases inflammation and does not inhibit the development of osteoarthritis in obese macrophage Fas-induced apoptosistransgenic mice. 2017. 69(9): p. 1772-1783.

50. Usher, K.M., et al., Pathological mechanisms and therapeutic outlooks for arthrofibrosis. 2019. 7(1): p. 1-24.

51. Paish, H.L., et al., Fibroblasts promote inflammation and pain via IL-1 $\alpha$ induction of the monocyte chemoattractant chemokine (CC motif) ligand 2. 2018. 188(3): p. 696-714.

52. Davies, L.C., et al., Distinct bone marrow-derived and tissue-resident macrophage lineages proliferate at key stages during inflammation. 2013. 4(1): p. 1-10.

53. Misharin, A.V., et al., Nonclassical Ly6C- monocytes drive the development of inflammatory arthritis in mice. 2014. 9(2): p. 591-604.

54. Wermuth, P.J., S.A.J.C. Jimenez, and t. medicine, The significance of macrophage polarization subtypes for animal models of tissue fibrosis and human fibrotic diseases. 2015. 4(1): p. 1-19.

55. Wynn, T.A. and K.M.J.I. Vannella, Macrophages in tissue repair, regeneration, and fibrosis. 2016. 44(3): p. 450-462.

56. Witherel, C.E., et al., Macrophage and Fibroblast Interactions in Biomaterial-Mediated Fibrosis. 2019. 8(4): p. 1801451.

57. Rőszer, T.J.M.o.i., Understanding the mysterious M2 macrophage through activation markers and effector mechanisms. 2015. 2015.

58. Braga, T.T., J.S.H. Agudelo, and N.O.S.J.F.i.i. Camara, Macrophages during the fibrotic process: $M 2$ as friend and foe. 2015. 6: p. 602.

59. Ambarus, C.A., et al., Intimal lining layer macrophages but not synovial sublining macrophages display an IL-10 polarized-like phenotype in chronic synovitis. 2012. 14(2): p. R74.

60. Huang, Q.Q., et al., Association of Increased F4/80high Macrophages With Suppression of Serum-Transfer Arthritis in Mice With Reduced FLIP in Myeloid Cells. 2017. 69(9): p. 1762-1771. 
61. Daghestani, H.N., et al., Soluble macrophage biomarkers indicate inflammatory phenotypes in patients with knee osteoarthritis. 2015. 67(4): p. 956-965.

62. Insel, P.A., et al., cAMP and Epac in the regulation of tissue fibrosis. 2012. 166(2): p. 447456.

63. Liu, X., et al., cAMP inhibits transforming growth factor- $\beta$-stimulated collagen synthesis via inhibition of extracellular signal-regulated kinase 1/2 and Smad signaling in cardiac fibroblasts. 2006. 70(6): p. 1992-2003.

64. Liu, X., et al., Fibrotic lung fibroblasts show blunted inhibition by cAMP due to deficient cAMP response element-binding protein phosphorylation. 2005. 315(2): p. 678-687.

65. Sapio, L., et al., The natural cAMP elevating compound forskolin in cancer therapy: is it time? 2017. 232(5): p. 922-927.

66. Seamon, K.B., W. Padgett, and J.W.J.P.o.t.N.A.o.S. Daly, Forskolin: unique diterpene activator of adenylate cyclase in membranes and in intact cells. 1981. 78(6): p. 3363-3367.

67. Häfner, S., et al., Mechanism of inhibition of Raf-1 by protein kinase A. 1994. 14(10): p. 6696-6703.

68. Cass, L.A., et al., Protein kinase A-dependent and-independent signaling pathways contribute to cyclic AMP-stimulated proliferation. 1999. 19(9): p. 5882-5891.

69. Jay, G.D., D.E. Britt, and C.J.T.J.o.r. Cha, Lubricin is a product of megakaryocyte stimulating factor gene expression by human synovial fibroblasts. 2000. 27(3): p. 594-600.

70. Jay, G.D., K. Haberstroh, and C.J.J.J.o.b.m.r. Cha, Comparison of the boundarylubricating ability of bovine synovial fluid, lubricin, and Healon. 1998. 40(3): p. 414-418.

71. Jay, G.D., et al., Homology of lubricin and superficial zone protein (SZP): products of megakaryocyte stimulating factor (MSF) gene expression by human synovial fibroblasts and articular chondrocytes localized to chromosome 1q25. 2001. 19(4): p. 677-687.

72. Swann, D., et al., The molecular structure and lubricating activity of lubricin isolated from bovine and human synovial fluids. 1985. 225(1): p. 195-201.

73. Catterall, J.B., et al., Changes in serum and synovial fluid biomarkers after acute injury (NCT00332254). 2010. 12(6): p. R229.

74. Elsaid, K., et al., Decreased lubricin concentrations and markers of joint inflammation in the synovial fluid of patients with anterior cruciate ligament injury. 2008. 58(6): p. 17071715.

75. Elsaid, K., et al., The impact of anterior cruciate ligament injury on lubricin metabolism and the effect of inhibiting tumor necrosis factor $\alpha$ on chondroprotection in an animal model. 2009. 60(10): p. 2997-3006. 
76. Kosinska, M.K., et al., Articular joint lubricants during osteoarthritis and rheumatoid arthritis display altered levels and molecular species. 2015. 10(5).

77. Young, A.A., et al., Proteoglycan 4 downregulation in a sheep meniscectomy model of early osteoarthritis. 2006. 8(2): p. R41.

78. Jones, A. and C.J.E.C.M. Flannery, Bioregulation of lubricin expression by growth factors and cytokines. 2007. 13(1): p. 40-45.

79. McNary, S.M., K.A. Athanasiou, and A.H.J.T.E.P.A. Reddi, Transforming growth factor $\beta$-induced superficial zone protein accumulation in the surface zone of articular cartilage is dependent on the cytoskeleton. 2014. 20(5-6): p. 921-929.

80. Blewis, M.E., et al., Interactive cytokine regulation of synoviocyte lubricant secretion. 2010. 16(4): p. 1329-1337.

81. Rhee, D.K., et al., Consequences of disease-causing mutations on lubricin protein synthesis, secretion, and post-translational processing. 2005. 280(35): p. 31325-31332.

82. Jay, G.D., et al., Association between friction and wear in diarthrodial joints lacking lubricin. 2007. 56(11): p. 3662-3669.

83. Jay, G.D., D.A. Harris, and C.-J.J.G.j. Cha, Boundary lubrication by lubricin is mediated by O-linked $\beta$ (1-3) Gal-GalNAc oligosaccharides. 2001. 18(10): p. 807-815.

84. Estrella, R.P., et al., The glycosylation of human synovial lubricin: implications for its role in inflammation. 2010. 429(2): p. 359-367.

85. Jin, C., et al., Human synovial lubricin expresses sialyl Lewis $x$ determinant and has Lselectin ligand activity. 2012. 287(43): p. 35922-35933.

86. Al-Sharif, A., et al., Lubricin/proteoglycan 4 binding to CD44 receptor: a mechanism of the suppression of proinflammatory cytokine-induced synoviocyte proliferation by lubricin. 2015. 67(6): p. 1503-1513.

87. Temple-Wong, M.M., et al., Hyaluronan concentration and size distribution in human knee synovial fluid: variations with age and cartilage degeneration. 2016. 18(1): p. 18.

88. Yang, Y.M., et al., Hyaluronan synthase 2-mediated hyaluronan production mediates Notch1 activation and liver fibrosis. 2019. 11(496): p. eaat9284.

89. Culty, M., H.A. Nguyen, and C.B.J.T.J.o.c.b. Underhill, The hyaluronan receptor (CD44) participates in the uptake and degradation of hyaluronan. 1992. 116(4): p. 1055-1062.

90. Wang, C.-T., et al., High molecular weight hyaluronic acid down-regulates the gene expression of osteoarthritis-associated cytokines and enzymes in fibroblast-like synoviocytes from patients with early osteoarthritis. 2006. 14(12): p. 1237-1247. 
91. Campo, G.M., et al., Hyaluronan reduces inflammation in experimental arthritis by modulating TLR-2 and TLR-4 cartilage expression. 2011. 1812(9): p. 1170-1181.

92. Plaas, A., et al., Intraarticular injection of hyaluronan prevents cartilage erosion, periarticular fibrosis and mechanical allodynia and normalizes stance time in murine knee osteoarthritis. 2011. 13(2): p. R46.

93. Ponta, H., L. Sherman, and P.A.J.N.r.M.c.b. Herrlich, CD44: from adhesion molecules to signalling regulators. 2003. 4(1): p. 33-45.

94. Senbanjo, L.T., M.A.J.F.i.c. Chellaiah, and d. biology, CD44: a multifunctional cell surface adhesion receptor is a regulator of progression and metastasis of cancer cells. 2017. 5: p. 18.

95. Fuchs, S., et al., Expression of the CD44 variant isoform 5 in the human osteoarthritic knee joint: correlation with radiological, histomorphological, and biochemical parameters. 2004. 22(4): p. 774-780.

96. Rothman, B., et al., Elevation of intracellular cAMP in human T lymphocytes by an antiCD44 mAb. 1993. 151(11): p. 6036-6042.

97. Amash, A., et al., CD44 antibody inhibition of macrophage phagocytosis targets $F_{c} \gamma$ receptor-and complement receptor 3-dependent mechanisms. 2016. 196(8): p. 3331-3340.

98. Liang, J., et al., CD44 is a negative regulator of acute pulmonary inflammation and lipopolysaccharide-TLR signaling in mouse macrophages. 2007. 178(4): p. 2469-2475.

99. Racine, R.R., et al., CD44 induced enhancement of phosphatase activity and calcium influx: Modifications of EGR-1 expression and cell proliferation. 2016. 6: p. 172-178.

100. Acharya, P.S., et al., Fibroblast migration is mediated by CD44-dependent TGF $\beta$ activation. 2008. 121(9): p. 1393-1402.

101. Costanza, B., et al., Stromal modulators of TGF- $\beta$ in cancer. 2017. 6(1): p. 7.

102. Ropert, C., B.S. Franklin, and R.T. Gazzinelli. Role of TLRs/MyD88 in host resistance and pathogenesis during protozoan infection: lessons from malaria. in Seminars in Immunopathology. 2008. Springer.

103. Chen, D., et al., Osteoarthritis: toward a comprehensive understanding of pathological mechanism. Bone research, 2017. 5(1): p. 1-13.

104. Loeser, R.F., et al., Osteoarthritis: a disease of the joint as an organ. Arthritis \& Rheumatism, 2012. 64(6): p. 1697-1707.

105. Felson, D.T., et al., Obesity and knee osteoarthritis: the Framingham Study. Annals of internal medicine, 1988. 109(1): p. 18-24. 
106. Felson, D.T., et al., Risk factors for incident radiographic knee osteoarthritis in the elderly. The Framingham Study. Arthritis \& Rheumatism: Official Journal of the American College of Rheumatology, 1997. 40(4): p. 728-733.

107. Robinson, W.H., et al., Low-grade inflammation as a key mediator of the pathogenesis of osteoarthritis. Nature Reviews Rheumatology, 2016. 12(10): p. 580-592.

108. Roos, E.M., Joint injury causes knee osteoarthritis in young adults. Current opinion in rheumatology, 2005. 17(2): p. 195-200.

109. Thijssen, E., A. van Caam, and P.M. van der Kraan, Obesity and osteoarthritis, more than just wear and tear: pivotal roles for inflamed adipose tissue and dyslipidaemia in obesityinduced osteoarthritis. Rheumatology, 2015. 54(4): p. 588-600.

110. Hill, C.L., et al., Synovitis detected on magnetic resonance imaging and its relation to pain and cartilage loss in knee osteoarthritis. Annals of the rheumatic diseases, 2007. 66(12): p. 1599-1603.

111. Ishijima, M., et al., Relationships between biomarkers of cartilage, bone, synovial metabolism and knee pain provide insights into the origins of pain in early knee osteoarthritis. Arthritis research \& therapy, 2011. 13(1): p. R22.

112. Mathiessen, A. and P.G. Conaghan, Synovitis in osteoarthritis: current understanding with therapeutic implications. Arthritis research \& therapy, 2017. 19(1): p. 18.

113. Roemer, F.W., et al., Presence of MRI-detected joint effusion and synovitis increases the risk of cartilage loss in knees without osteoarthritis at 30-month follow-up: the MOST study. Annals of the rheumatic diseases, 2011. 70(10): p. 1804-1809.

114. Torres, L., et al., The relationship between specific tissue lesions and pain severity in persons with knee osteoarthritis. Osteoarthritis and cartilage, 2006. 14(10): p. 1033-1040.

115. Smith, M.D., Suppl 1: The Normal Synovium. The open rheumatology journal, 2011. 5: p. 100.

116. Smith, M., et al., Microarchitecture and protective mechanisms in synovial tissue from clinically and arthroscopically normal knee joints. Annals of the rheumatic diseases, 2003. 62(4): p. 303-307.

117. De Lange-Brokaar, B.J., et al., Synovial inflammation, immune cells and their cytokines in osteoarthritis: a review. Osteoarthritis and cartilage, 2012. 20(12): p. 1484-1499.

118. Scanzello, C.R. and S.R. Goldring, The role of synovitis in osteoarthritis pathogenesis. Bone, 2012. 51(2): p. 249-257.

119. Haynes, M.K., E.L. Hume, and J.B. Smith, Phenotypic characterization of inflammatory cells from osteoarthritic synovium and synovial fluids. Clinical Immunology, 2002. 105(3): p. 315-325. 
120. Saito, I., et al., Increased cellular infiltrate in inflammatory synovia of osteoarthritic knees. Osteoarthritis and cartilage, 2002. 10(2): p. 156-162.

121. Bondeson, J., et al., The role of synovial macrophages and macrophage-produced mediators in driving inflammatory and destructive responses in osteoarthritis. Arthritis \& Rheumatism, 2010. 62(3): p. 647-657.

122. Bondeson, J., et al., The role of synovial macrophages and macrophage-produced cytokines in driving aggrecanases, matrix metalloproteinases, and other destructive and inflammatory responses in osteoarthritis. Arthritis research \& therapy, 2006. 8(6): p. R187.

123. Blom, A.B., et al., Synovial lining macrophages mediate osteophyte formation during experimental osteoarthritis. Osteoarthritis and cartilage, 2004. 12(8): p. 627-635.

124. Blom, A.B., et al., Crucial role of macrophages in matrix metalloproteinase-mediated cartilage destruction during experimental osteoarthritis: involvement of matrix metalloproteinase 3. Arthritis \& Rheumatism, 2007. 56(1): p. 147-157.

125. Orlowsky, E.W. and V.B. Kraus, The role of innate immunity in osteoarthritis: when our first line of defense goes on the offensive. The Journal of rheumatology, 2015. 42(3): p. 363-371.

126. Sokolove, J. and C.M. Lepus, Role of inflammation in the pathogenesis of osteoarthritis: latest findings and interpretations. Therapeutic advances in musculoskeletal disease, 2013. 5(2): p. 77-94.

127. Culty, M., H.A. Nguyen, and C.B. Underhill, The hyaluronan receptor (CD44) participates in the uptake and degradation of hyaluronan. The Journal of cell biology, 1992. 116(4): p. 1055-1062.

128. Kataoka, Y., et al., Mechanisms involved in suppression of ADAMTS4 expression in synoviocytes by high molecular weight hyaluronic acid. Biochemical and biophysical research communications, 2013. 432(4): p. 580-585.

129. Wang, C.-T., et al., High molecular weight hyaluronic acid down-regulates the gene expression of osteoarthritis-associated cytokines and enzymes in fibroblast-like synoviocytes from patients with early osteoarthritis. Osteoarthritis and cartilage, 2006. 14(12): p. 1237-1247.

130. Campo, G.M., et al., Hyaluronan reduces inflammation in experimental arthritis by modulating TLR-2 and TLR-4 cartilage expression. Biochimica et Biophysica Acta (BBA)Molecular Basis of Disease, 2011. 1812(9): p. 1170-1181.

131. Park, E., et al., Optimized THP-1 differentiation is required for the detection of responses to weak stimuli. Inflammation research, 2007. 56(1): p. 45-50. 
132. Tsolmongyn, B., et al., A Toll-like receptor 2 ligand, P am 3 CSK 4, augments interferon$\gamma$-induced nitric oxide production via a physical association between MyD88 and interferon- $\gamma$ receptor in vascular endothelial cells. Immunology, 2013. 140(3): p. 352-361.

133. Knopick, P.L. and D.S. Bradley, Detecting immune responses to type III secretion systems, in Type 3 Secretion Systems. 2017, Springer. p. 165-172.

134. Livak, K.J. and T.D. Schmittgen, Analysis of relative gene expression data using real-time quantitative PCR and the 2- $\triangle \triangle C T$ method. methods, 2001. 25(4): p. 402-408.

135. Protin, U., et al., CD44-deficient mice develop normally with changes in subpopulations and recirculation of lymphocyte subsets. The Journal of Immunology, 1999. 163(9): p. 4917-4923.

136. Zhang, X., R. Goncalves, and D.M. Mosser, The isolation and characterization of murine macrophages. Current protocols in immunology, 2008. 83(1): p. 14.1. 1-14.1. 14.

137. Ozawa, T., et al., Thymic stromal lymphopoietin secretion of synovial fibroblasts is positively and negatively regulated by Toll-like receptors/nuclear factor- $\kappa B$ pathway and interferon- $\gamma /$ dexamethasone. Modern rheumatology, 2007. 17(6): p. 459-463.

138. Pazár, B., et al., Basic calcium phosphate crystals induce monocyte/macrophage IL-1 $\beta$ secretion through the NLRP3 inflammasome in vitro. The Journal of Immunology, 2011. 186(4): p. 2495-2502.

139. Gómez, R., et al., TLR4 signalling in osteoarthritis-finding targets for candidate $D M O A D s$. Nature Reviews Rheumatology, 2015. 11(3): p. 159.

140. Scanzello, C.R., A. Plaas, and M.K. Crow, Innate immune system activation in osteoarthritis: is osteoarthritis a chronic wound? Current opinion in rheumatology, 2008. 20(5): p. 565-572.

141. Kraus, V.B., et al., Direct in vivo evidence of activated macrophages in human osteoarthritis. Osteoarthritis and cartilage, 2016. 24(9): p. 1613-1621.

142. Parker, L.C., et al., Toll-like receptor (TLR) 2 and TLR4 agonists regulate CCR expression in human monocytic cells. The Journal of Immunology, 2004. 172(8): p. 4977-4986.

143. Ponta, H., L. Sherman, and P.A. Herrlich, CD44: from adhesion molecules to signalling regulators. Nature reviews Molecular cell biology, 2003. 4(1): p. 33-45.

144. Amash, A., et al., CD44 antibody inhibition of macrophage phagocytosis targets Fc $\gamma$ receptor-and complement receptor 3-dependent mechanisms. The Journal of Immunology, 2016. 196(8): p. 3331-3340.

145. Kawana, H., et al., CD44 suppresses TLR-mediated inflammation. The Journal of Immunology, 2008. 180(6): p. 4235-4245. 
146. Liang, J., et al., CD44 is a negative regulator of acute pulmonary inflammation and lipopolysaccharide-TLR signaling in mouse macrophages. The Journal of Immunology, 2007. 178(4): p. 2469-2475.

147. Shanley, T.P., et al., The serine/threonine phosphatase, PP2A: endogenous regulator of inflammatory cell signaling. The Journal of Immunology, 2001. 166(2): p. 966-972.

148. Sun, L., et al., Study of protein phosphatase 2A (PP2A) activity in LPS-induced tolerance using fluorescence-based and immunoprecipitation-aided methodology. Biomolecules, 2015. 5(3): p. 1284-1301.

149. Racine, R.R., et al., CD44 induced enhancement of phosphatase activity and calcium influx: Modifications of EGR-1 expression and cell proliferation. Biochemistry and biophysics reports, 2016. 6: p. 172-178.

150. Kosinska, M.K., et al., Articular joint lubricants during osteoarthritis and rheumatoid arthritis display altered levels and molecular species. PloS one, 2015. 10(5).

151. Alquraini, A., et al., The interaction of lubricin/proteoglycan 4 (PRG4) with toll-like receptors 2 and 4: an anti-inflammatory role of PRG4 in synovial fluid. Arthritis research $\&$ therapy, 2015. 17(1): p. 353.

152. Nair, A., et al., Synovial fluid from patients with early osteoarthritis modulates fibroblastlike synoviocyte responses to Toll-like receptor 4 and Toll-like receptor 2 ligands via soluble CD14. Arthritis \& Rheumatism, 2012. 64(7): p. 2268-2277.

153. Sohn, D.H., et al., Plasma proteins present in osteoarthritic synovial fluid can stimulate cytokine production via Toll-like receptor 4. Arthritis research \& therapy, 2012. 14(1): p. R7.

154. Al-Sharif, A., et al., Lubricin/proteoglycan 4 binding to CD44 receptor: a mechanism of the suppression of proinflammatory cytokine-induced synoviocyte proliferation by lubricin. Arthritis \& rheumatology, 2015. 67(6): p. 1503-1513.

155. Alquraini, A., et al., The autocrine role of proteoglycan-4 (PRG4) in modulating osteoarthritic synoviocyte proliferation and expression of matrix degrading enzymes. Arthritis research \& therapy, 2017. 19(1): p. 89.

156. Martell-Pelletier, J., et al., Osteoarthritis. Nature Reviews Disease Primers, 2016. 2.

157. Benito, M.J., et al., Synovial tissue inflammation in early and late osteoarthritis. Annals of the rheumatic diseases, 2005. 64(9): p. 1263-1267.

158. Sellam, J. and F. Berenbaum, The role of synovitis in pathophysiology and clinical symptoms of osteoarthritis. Nature Reviews Rheumatology, 2010. 6(11): p. 625.

159. Smith, M.D., et al., Synovial membrane inflammation and cytokine production in patients with early osteoarthritis. The Journal of rheumatology, 1997. 24(2): p. 365-371. 
160. Wenham, C.Y. and P.G. Conaghan, The role of synovitis in osteoarthritis. Therapeutic advances in musculoskeletal disease, 2010. 2(6): p. 349-359.

161. Loeuille, D., et al., Macroscopic and microscopic features of synovial membrane inflammation in the osteoarthritic knee: correlating magnetic resonance imaging findings with disease severity. Arthritis \& Rheumatism, 2005. 52(11): p. 3492-3501.

162. Van Der Kraan, P.M., The changing role of TGF $\beta$ in healthy, ageing and osteoarthritic joints. Nature Reviews Rheumatology, 2017. 13(3): p. 155.

163. Remst, D., et al., Osteoarthritis-related fibrosis is associated with both elevated pyridinoline cross-link formation and lysyl hydroxylase $2 b$ expression. Osteoarthritis and cartilage, 2013. 21(1): p. 157-164.

164. Remst, D., et al., Gene Expression Analysis of Murine and Human Osteoarthritis Synovium Reveals Elevation of Transforming Growth Factor $\beta$-Responsive Genes in OsteoarthritisRelated Fibrosis. Arthritis \& rheumatology, 2014. 66(3): p. 647-656.

165. Shen, J., S. Li, and D. Chen, TGF- $\beta$ signaling and the development of osteoarthritis. Bone research, 2014. 2: p. 14002.

166. Mattey, D., et al., Transforming growth factor $\beta 1$ and interleukin 4 induced $\alpha$ smooth muscle actin expression and myofibroblast-like differentiation in human synovial fibroblasts in vitro: modulation by basic fibroblast growth factor. Annals of the rheumatic diseases, 1997. 56(7): p. 426-431.

167. Weigel, P.H. and P.L. DeAngelis, Hyaluronan synthases: a decade-plus of novel glycosyltransferases. Journal of Biological Chemistry, 2007. 282(51): p. 36777-36781.

168. Flannery, C.R., et al., Articular cartilage superficial zone protein (SZP) is homologous to megakaryocyte stimulating factor precursor and is a multifunctional proteoglycan with potential growth-promoting, cytoprotective, and lubricating properties in cartilage metabolism. Biochemical and biophysical research communications, 1999. 254(3): p. 535541.

169. Jay, G.D., D.E. Britt, and C. Cha, Lubricin is a product of megakaryocyte stimulating factor gene expression by human synovial fibroblasts. The Journal of rheumatology, 2000. 27(3): p. 594-600.

170. Ludwig, T.E., M.M. Hunter, and T.A. Schmidt, Cartilage boundary lubrication synergism is mediated by hyaluronan concentration and PRG4 concentration and structure. BMC musculoskeletal disorders, 2015. 16(1): p. 386.

171. Heusinger-Ribeiro, J., et al., Expression of connective tissue growth factor in human renal fibroblasts: regulatory roles of RhoA and cAMP. Journal of the American Society of Nephrology, 2001. 12(9): p. 1853-1861. 
172. Sachs, B.D., et al., $p 75$ neurotrophin receptor regulates tissue fibrosis through inhibition of plasminogen activation via a PDE4/cAMP/PKA pathway. The Journal of cell biology, 2007. 177(6): p. 1119-1132.

173. Selige, J., et al., Cytokine-dependent balance of mitogenic effects in primary human lung fibroblasts related to cyclic AMP signaling and phosphodiesterase 4 inhibition. Journal of cellular physiology, 2010. 223(2): p. 317-326.

174. Yokoyama, U., et al., The cyclic AMP effector Epac integrates pro-and anti-fibrotic signals. Proceedings of the National Academy of Sciences, 2008. 105(17): p. 6386-6391.

175. Haas, S. and R.H. Straub, Disruption of rhythms of molecular clocks in primary synovial fibroblasts of patients with osteoarthritis and rheumatoid arthritis, role of IL-1B/TNF. Arthritis research \& therapy, 2012. 14(3): p. R122.

176. Neumann, E., et al., Cell culture and passaging alters gene expression pattern and proliferation rate in rheumatoid arthritis synovial fibroblasts. Arthritis research \& therapy, 2010. 12(3): p. R83.

177. Liu, X., et al., cAMP inhibits transforming growth factor- $\beta$-stimulated collagen synthesis via inhibition of extracellular signal-regulated kinase 1/2 and Smad signaling in cardiac fibroblasts. Molecular pharmacology, 2006. 70(6): p. 1992-2003.

178. Törrönen, K., et al., Tissue distribution and subcellular localization of hyaluronan synthase isoenzymes. Histochemistry and cell biology, 2014. 141(1): p. 17-31.

179. Jay, G.D., et al., Prevention of cartilage degeneration and restoration of chondroprotection by lubricin tribosupplementation in the rat following anterior cruciate ligament transection. Arthritis \& Rheumatism, 2010. 62(8): p. 2382-2391.

180. Rhee, D.K., et al., The secreted glycoprotein lubricin protects cartilage surfaces and inhibits synovial cell overgrowth. The Journal of clinical investigation, 2005. 115(3): p. 622-631.

181. Bastiaansen-Jenniskens, Y.M., et al., Stimulation of fibrotic processes by the infrapatellar fat pad in cultured synoviocytes from patients with osteoarthritis: a possible role for prostaglandin f2 $\alpha$. Arthritis \& Rheumatism, 2013. 65(8): p. 2070-2080.

182. Murakami, S., et al., Quantitative analysis of synovial fibrosis in the infrapatellar fat pad before and after anterior cruciate ligament reconstruction. The American journal of sports medicine, 1997. 25(1): p. 29-34.

183. Remst, D.F., et al., TGF-ss induces Lysyl hydroxylase $2 b$ in human synovial osteoarthritic fibroblasts through ALK5 signaling. Cell and tissue research, 2014. 355(1): p. 163-171.

184. Blewis, M.E., et al., Interactive cytokine regulation of synoviocyte lubricant secretion. Tissue Engineering Part A, 2010. 16(4): p. 1329-1337. 
185. Inoue, H., et al., An investigation of cell proliferation and soluble mediators induced by interleukin $1 \beta$ in human synovial fibroblasts: comparative response in osteoarthritis and rheumatoid arthritis. Inflammation Research, 2001. 50(2): p. 65-72.

186. Seamon, K.B., W. Padgett, and J.W. Daly, Forskolin: unique diterpene activator of adenylate cyclase in membranes and in intact cells. Proceedings of the National Academy of Sciences, 1981. 78(6): p. 3363-3367.

187. Sapio, L., et al., The natural cAMP elevating compound forskolin in cancer therapy: is it time? Journal of cellular physiology, 2017. 232(5): p. 922-927.

188. Cass, L.A., et al., Protein kinase A-dependent and-independent signaling pathways contribute to cyclic AMP-stimulated proliferation. Molecular and Cellular Biology, 1999. 19(9): p. 5882-5891.

189. Chaponnier, C. and G. Gabbiani, Pathological situations characterized by altered actin isoform expression. The Journal of Pathology: A Journal of the Pathological Society of Great Britain and Ireland, 2004. 204(4): p. 386-395.

190. Ehrlich, H., G. Allison, and M. Leggett, The myofibroblast, cadherin, a smooth muscle actin and the collagen effect. Cell Biochemistry and Function: Cellular biochemistry and its modulation by active agents or disease, 2006. 24(1): p. 63-70.

191. Steenvoorden, M.M., et al., Transition of healthy to diseased synovial tissue in rheumatoid arthritis is associated with gain of mesenchymal/fibrotic characteristics. Arthritis research \& therapy, 2006. 8(6): p. R165.

192. Sasabe, R., et al., Effects of joint immobilization on changes in myofibroblasts and collagen in the rat knee contracture model. Journal of Orthopaedic Research, 2017. 35(9): p. 19982006.

193. RECKLIES, A.D., et al., Differential regulation and expression of hyaluronan synthases in human articular chondrocytes, synovial cells and osteosarcoma cells. Biochemical Journal, 2001. 354(1): p. 17-24.

194. Ogawa, H., et al., Mechanical motion promotes expression of Prg4 in articular cartilage via multiple CREB-dependent, fluid flow shear stress-induced signaling pathways. Genes \& development, 2014. 28(2): p. 127-139.

195. Jay, G.D. and K.A. Waller, The biology of lubricin: near frictionless joint motion. Matrix Biology, 2014. 39: p. 17-24.

196. Ikegawa, S., et al., Isolation, characterization and mapping of the mouse and human PRG4 (proteoglycan 4) genes. Cytogenetic and Genome Research, 2000. 90(3-4): p. 291-297.

197. Schmidt, T.A., A.H. Plaas, and J.D. Sandy, Disulfide-bonded multimers of proteoglycan 4 (PRG4) are present in normal synovial fluids. Biochimica et Biophysica Acta (BBA)General Subjects, 2009. 1790(5): p. 375-384. 
198. Zappone, B., et al., Adsorption, lubrication, and wear of lubricin on model surfaces: polymer brush-like behavior of a glycoprotein. Biophysical journal, 2007. 92(5): p. 16931708.

199. Cross, M., et al., The global burden of hip and knee osteoarthritis: estimates from the global burden of disease 2010 study. Annals of the rheumatic diseases, 2014. 73(7): p. 1323-1330.

200. Fu, K., S.R. Robbins, and J.J. McDougall, Osteoarthritis: the genesis of pain. Rheumatology, 2018.57(suppl_4): p. iv43-iv50.

201. Kloppenburg, M. and F. Berenbaum, Osteoarthritis year in review 2019: epidemiology and therapy. Osteoarthritis and Cartilage, 2020. 28(3): p. 242-248.

202. Cleveland, R., et al., The impact of painful knee osteoarthritis on mortality: a communitybased cohort study with over 24 years of follow-up. Osteoarthritis and cartilage, 2019. 27(4): p. 593-602.

203. Veronese, N., et al. Osteoarthritis and mortality: a prospective cohort study and systematic review with meta-analysis. in Seminars in arthritis and rheumatism. 2016. Elsevier.

204. Wang, Y., et al., Knee osteoarthritis, potential mediators, and risk of all-cause mortality: data from the Osteoarthritis Initiative. Arthritis Care \& Research, 2020.

205. Carbone, A. and S. Rodeo, Review of current understanding of post-traumatic osteoarthritis resulting from sports injuries. Journal of orthopaedic research, 2017. 35(3): p. 397-405.

206. O'Neill, T.W., P.S. McCabe, and J. McBeth, Update on the epidemiology, risk factors and disease outcomes of osteoarthritis. Best practice \& research Clinical rheumatology, 2018. 32(2): p. 312-326.

207. Poulsen, E., et al., Knee osteoarthritis risk is increased 4-6 fold after knee injury-a systematic review and meta-analysis. British journal of sports medicine, 2019. 53(23): p. 1454-1463.

208. Silverwood, V., et al., Current evidence on risk factors for knee osteoarthritis in older adults: a systematic review and meta-analysis. Osteoarthritis and cartilage, 2015. 23(4): p. 507-515.

209. Poole, A.R., Osteoarthritis as a whole joint disease. HSS journal, 2012. 8(1): p. 4-6.

210. Baker, K., et al., Relation of synovitis to knee pain using contrast-enhanced MRIs. Annals of the rheumatic diseases, 2010. 69(10): p. 1779-1783.

211. Guermazi, A., et al., Synovitis in knee osteoarthritis assessed by contrast-enhanced magnetic resonance imaging (MRI) is associated with radiographic tibiofemoral 
osteoarthritis and MRI-detected widespread cartilage damage: the MOST study. The Journal of rheumatology, 2014. 41(3): p. 501-508.

212. Gómez, R., et al., TLR4 signalling in osteoarthritis-finding targets for candidate DMOADs. Nature Reviews Rheumatology, 2015. 11(3): p. 159-170.

213. Herrero-Beaumont, G., et al., Targeting chronic innate inflammatory pathways, the main road to prevention of osteoarthritis progression. Biochemical Pharmacology, 2019. 165: p. 24-32.

214. Kalaitzoglou, E., T.M. Griffin, and M.B. Humphrey, Innate immune responses and osteoarthritis. Current Rheumatology Reports, 2017. 19(8): p. 45.

215. Sharma, N., et al., Inflammation and joint destruction may be linked to the generation of cartilage metabolites of ADAMTS-5 through activation of toll-like receptors. Osteoarthritis and Cartilage, 2019.

216. Conaghan, P., et al., Clinical and ultrasonographic predictors of joint replacement for knee osteoarthritis: results from a large, 3-year, prospective EULAR study. Annals of the rheumatic diseases, 2010. 69(4): p. 644-647.

217. Jay, G.D., D.E. Britt, and C. Cha, Lubricin is a product of megakaryocyte stimulating factor gene expression by human synovial fibroblasts. The Journal of rheumatology, 2000. 27(3): p. 594.

218. Abdul, N., et al., Fibrosis is a common outcome following total knee arthroplasty. Scientific reports, 2015. 5: p. 16469.

219. Bong, M.R. and P.E. Di Cesare, Stiffness after total knee arthroplasty. JAAOS-Journal of the American Academy of Orthopaedic Surgeons, 2004. 12(3): p. 164-171.

220. Qadri, M.M., et al., cAMP attenuates TGF- $\beta$ 's profibrotic responses in osteoarthritic synoviocytes: involvement of hyaluronan and PRG4. American Journal of Physiology-Cell Physiology, 2018. 315(3): p. C432-C443.

221. Samsom, M.L., et al., Characterization of full-length recombinant human Proteoglycan 4 as an ocular surface boundary lubricant. Experimental eye research, 2014. 127: p. 14-19.

222. Acharya, P.S., et al., Fibroblast migration is mediated by CD44-dependent TGF $\beta$ activation. Journal of cell science, 2008. 121(9): p. 1393-1402.

223. Ascione, F., et al., Comparison between fibroblast wound healing and cell random migration assays in vitro. Experimental cell research, 2016. 347(1): p. 123-132.

224. Hill, A., et al., Lubricin restoration in a mouse model of congenital deficiency. Arthritis \& rheumatology, 2015. 67(11): p. 3070-3081. 
225. Jay, G.D., K. Haberstroh, and C.J. Cha, Comparison of the boundary-lubricating ability of bovine synovial fluid, lubricin, and Healon. Journal of biomedical materials research, 1998. 40(3): p. 414-418.

226. Swann, D., et al., The molecular structure and lubricating activity of lubricin isolated from bovine and human synovial fluids. Biochemical Journal, 1985. 225(1): p. 195-201.

227. Jay, G.D., et al., Homology of lubricin and superficial zone protein (SZP): products of megakaryocyte stimulating factor (MSF) gene expression by human synovial fibroblasts and articular chondrocytes localized to chromosome 1q25. Journal of Orthopaedic Research, 2001. 19(4): p. 677-687.

228. Larson, K.M., et al., Early genetic restoration of lubricin expression in transgenic mice mitigates chondrocyte peroxynitrite release and caspase-3 activation. Osteoarthritis and cartilage, 2017. 25(9): p. 1488-1495.

229. Schmidt, T.A., et al., Boundary lubrication of articular cartilage: role of synovial fluid constituents. Arthritis \& Rheumatism, 2007. 56(3): p. 882-891.

230. Waller, K.A., L.X. Zhang, and G.D. Jay, Friction-induced mitochondrial dysregulation contributes to joint deterioration in Prg4 knockout mice. International Journal of Molecular Sciences, 2017. 18(6): p. 1252.

231. Elsaid, K., et al., The impact of anterior cruciate ligament injury on lubricin metabolism and the effect of inhibiting tumor necrosis factor $\alpha$ on chondroprotection in an animal model. Arthritis \& Rheumatism: Official Journal of the American College of Rheumatology, 2009. 60(10): p. 2997-3006.

232. Schmidt, T., et al., Differential regulation of proteoglycan 4 metabolism in cartilage by IL$1 \alpha, I G F-I$, and TGF- $\beta 1$. Osteoarthritis and cartilage, 2008. 16(1): p. 90-97.

233. Catterall, J.B., et al., Changes in serum and synovial fluid biomarkers after acute injury (NCT00332254). Arthritis research \& therapy, 2010. 12(6): p. R229.

234. Elsaid, K., et al., Decreased lubricin concentrations and markers of joint inflammation in the synovial fluid of patients with anterior cruciate ligament injury. Arthritis \& Rheumatism: Official Journal of the American College of Rheumatology, 2008. 58(6): p. 1707-1715.

235. Kosinska, M.K., et al., Articular joint lubricants during osteoarthritis and rheumatoid arthritis display altered levels and molecular species. PloS one, 2015. 10(5): p. e0125192.

236. Cui, Z., et al., Treatment with recombinant lubricin attenuates osteoarthritis by positive feedback loop between articular cartilage and subchondral bone in ovariectomized rats. Bone, 2015. 74: p. 37-47. 
237. Elsaid, K., et al., The impact of forced joint exercise on lubricin biosynthesis from articular cartilage following ACL transection and intra-articular lubricin's effect in exercised joints following ACL transection. Osteoarthritis and Cartilage, 2012. 20(8): p. 940-948.

238. Waller, K.A., et al., Intra-articular recombinant human proteoglycan 4 mitigates cartilage damage after destabilization of the medial meniscus in the Yucatan minipig. The American journal of sports medicine, 2017. 45(7): p. 1512-1521.

239. Senbanjo, L.T. and M.A. Chellaiah, CD44: a multifunctional cell surface adhesion receptor is a regulator of progression and metastasis of cancer cells. Frontiers in cell and developmental biology, 2017. 5: p. 18.

240. UNDERHILL, C., CD44: the hyaluronan receptor. Journal of cell science, 1992. 103(2): p. 293-298.

241. Weber, G.F., et al., Receptor-ligand interaction between CD44 and osteopontin (Eta-1). Science, 1996. 271(5248): p. 509-512.

242. Chen, C., et al., The biology and role of CD44 in cancer progression: therapeutic implications. Journal of hematology \& oncology, 2018. 11(1): p. 64.

243. Mellor, L., et al., Intracellular domain fragment of CD44 alters CD44 function in chondrocytes. Journal of Biological Chemistry, 2013. 288(36): p. 25838-25850.

244. Misra, S., et al., Interactions between hyaluronan and its receptors (CD44, RHAMM) regulate the activities of inflammation and cancer. Frontiers in immunology, 2015. 6: p. 201.

245. Julovi, S.M., et al., Inhibition of interleukin-1 $\beta$-stimulated production of matrix metalloproteinases by hyaluronan via CD44 in human articular cartilage. Arthritis \& Rheumatism: Official Journal of the American College of Rheumatology, 2004. 50(2): p. 516-525.

246. Yatabe, T., et al., Hyaluronan inhibits expression of ADAMTS4 (aggrecanase-1) in human osteoarthritic chondrocytes. Annals of the rheumatic diseases, 2009. 68(6): p. 1051-1058.

247. Plaas, A., et al., Intraarticular injection of hyaluronan prevents cartilage erosion, periarticular fibrosis and mechanical allodynia and normalizes stance time in murine knee osteoarthritis. Arthritis research \& therapy, 2011. 13(2): p. 1-14.

248. Waddell, D.D., et al., Hyaluronan Suppresses IL-1 [beta]-induced Metalloproteinase Activity from Synovial Tissue. Clinical Orthopaedics and Related Research (1976-2007), 2007. 465: p. 241-248.

249. Alquraini, A., et al., The interaction of lubricin/proteoglycan 4 (PRG4) with toll-like receptors 2 and 4: an anti-inflammatory role of PRG4 in synovial fluid. Arthritis research $\&$ therapy, 2015. 17(1): p. 1-12. 
250. Iqbal, S., et al., Lubricin/Proteoglycan 4 binds to and regulates the activity of Toll-Like Receptors In Vitro. Scientific reports, 2016. 6: p. 18910.

251. Qadri, M., et al., Recombinant human proteoglycan-4 reduces phagocytosis of urate crystals and downstream nuclear factor kappa $B$ and inflammasome activation and production of cytokines and chemokines in human and murine macrophages. Arthritis research \& therapy, 2018. 20(1): p. 1-16.

252. Wynn, T.A. and T.R. Ramalingam, Mechanisms of fibrosis: therapeutic translation for fibrotic disease. Nature medicine, 2012. 18(7): p. 1028.

253. Serini, G. and G. Gabbiani, Mechanisms of myofibroblast activity and phenotypic modulation. Experimental cell research, 1999. 250(2): p. 273-283.

254. Livne, A. and B. Geiger, The inner workings of stress fibers-from contractile machinery to focal adhesions and back. Journal of Cell Science, 2016. 129(7): p. 1293-1304.

255. Kim, D.H. and D. Wirtz, Focal adhesion size uniquely predicts cell migration. The FASEB Journal, 2013. 27(4): p. 1351-1361.

256. Keeley, E.C., B. Mehrad, and R.M. Strieter, Fibrocytes: bringing new insights into mechanisms of inflammation and fibrosis. The international journal of biochemistry \& cell biology, 2010. 42(4): p. 535-542.

257. Reilkoff, R.A., R. Bucala, and E.L. Herzog, Fibrocytes: emerging effector cells in chronic inflammation. Nature Reviews Immunology, 2011. 11(6): p. 427-435.

258. Kurowska-Stolarska, M. and S. Alivernini, Synovial tissue macrophages: friend or foe? RMD open, 2017. 3(2).

259. Bosch, U., et al., Arthrofibrosis is the result of a T cell mediated immune response. Knee Surgery, Sports Traumatology, Arthroscopy, 2001. 9(5): p. 282-289.

260. Freeman, T.A., et al., Reactive oxygen and nitrogen species induce protein and DNA modifications driving arthrofibrosis following total knee arthroplasty. Fibrogenesis \& Tissue Repair, 2009. 2(1): p. 5.

261. Donlin, L.T., et al., Modulation of TNF-induced macrophage polarization by synovial fibroblasts. The Journal of Immunology, 2014. 193(5): p. 2373-2383.

262. Wermuth, P.J. and S.A. Jimenez, The significance of macrophage polarization subtypes for animal models of tissue fibrosis and human fibrotic diseases. Clinical and translational medicine, 2015. 4(1): p. 1-19.

263. Biernacka, A., M. Dobaczewski, and N.G. Frangogiannis, TGF- $\beta$ signaling in fibrosis. Growth factors, 2011. 29(5): p. 196-202. 
264. Ruan, M.Z., et al., Proteoglycan 4 expression protects against the development of osteoarthritis. Science translational medicine, 2013. 5(176): p. 176ra34-176ra34.

265. Stone, A., et al., Combinatorial Prg4 and IL-1ra gene therapy protects against hyperalgesia and cartilage degeneration in post-traumatic osteoarthritis. Human gene therapy, 2019. 30(2): p. 225-235. 